\title{
Two-body scattering states in Minkowski space and the Nakanishi integral representation onto the null plane
}

\author{
T. Frederico ${ }^{a}$, G. Salmè $\grave{b}^{b}$ and M. Viviani ${ }^{c}$ \\ ${ }^{a}$ Dep. de Física, Instituto Tecnológico de Aeronáutica, \\ DCTA, 12.228-900 São José dos Campos, São Paulo, Brazil \\ ${ }^{b}$ Istituto Nazionale di Fisica Nucleare, \\ Sezione di Roma, P.le A. Moro 2, I-00185 Roma, Italy \\ ${ }^{c}$ Istituto Nazionale di Fisica Nucleare, Sezione di Pisa, \\ Largo Pontecorvo 3, 56100, Pisa, Italy
}

\begin{abstract}
The Nakanishi perturbative integral representation of the four-dimensional T-matrix is investigated in order to get a workable treatment for scattering states, solutions of the inhomogeneous Bethe-Salpeter Equation, in Minkowski space. The projection onto the null-plane of the fourdimensional inhomogeneous Bethe-Salpeter Equation plays a key role for devising an equation for the Nakanishi weight function (a real function), as in the homogeneous case that corresponds to bound states and it has been already studied within different frameworks. In this paper, the whole formal development is illustrated in detail and applied to a system, composed by two massive scalars interacting through the exchange of a massive scalar. The explicit expression of the scattering integral equations are also obtained in ladder approximation, and, as simple applications of our formalism, some limiting cases, like the zero-energy limit and the Wick-Cutkosky model in the continuum, are presented.
\end{abstract}

PACS numbers: 11.10.St,11.80.-m,03.65.Pm,03.65.Ge 


\section{INTRODUCTION}

The Bethe-Salpeter equation (BSE) [1] (see also [2] for an early and detailed review) is an important tool for investigating the non perturbative regime of many important issues in physics, within a relativistic framework. The huge tower of difficulties for obtaining solutions has been bravely faced with very different degrees of approximation and with different mathematical tools, e.g. by exploiting the classic Wick rotation trick [3] and free propagators (see e.g. Ref. [4]), by three-dimensional (3D) reductions (see, e.g., Ref. [5]) or by applying, in Euclidean space, the combination of Dyson-Schwinger and Bethe-Salpeter equations (see e.g. Ref. [6] for a recent review). The peculiar field of application is the description of bound states, but one can find attempts for solving also the scattering-state problem (see, e.g., [2, 7 9]). As mentioned above, the number of approaches for achieving solutions is quite large, and in this paper we focus on a particular scheme in Minkowski space. In order to avoid the well-known difficulties related to the cumbersome analytical behavior of the four-dimensional (4D) BSE, we adopt the framework proposed by Nakanishi in his seminal work [10], the so-called perturbation theory integral representation (PTIR) of i) the vertex function (that leads to the BSE for bound states), ii) the T-matrix (relevant for the present investigation of the scattering states) and iii) the general, multi-leg transition amplitudes. He carefully studied the parametric form of any Feynman diagram that contributes to the multi-leg transition amplitudes for interacting bosons, focusing on the denominator, since it governs the overall analytic behavior.

Nakanishi was able, through a clever change of variable, to give a common form to any denominator appearing in the parametric expressions, and then he formally summed up the infinite series of Feynman diagrams. The final result, aside from the convergence problem of the perturbation series [10], makes it possible to single out real, multi-variable weight functions (the Nakanishi amplitudes in what follows) modulating the denominator that generates the analytic behavior in Minkowski space of multi-leg transition amplitudes. It is noteworthy that a uniqueness theorem was proved for the PTIR transition amplitudes for bosonic systems [10].

Summarizing, PTIR yields a well-defined framework where classes of approximations to the multi-leg transition amplitude can be implemented in a systematic way, as shown, e.g.,

in Refs. [11, 12], where the BSE was investigated first within a ladder approximation of the 
kernel and then the cross-ladder class of diagrams was added. One could wonder if PTIR be able to deal with the analytic behavior of the non perturbative multi-leg amplitudes. For the scattering case, typical singularities like cuts, are certainly present in PTIR. For the bound states, one is interested in describing the vertex functions, which is related to the residue of the T-matrix at the bound-state poles. Moreover, numerical investigations performed till now [11 13] have shown that PTIR is able to accurately obtain both boundstate BS amplitude and corresponding masses. This strongly indicates [14] that solutions of the BSE, i. e. eigenfunctions obtained in a non perturbative framework, can be put in the form suggested by Nakanishi PTIR. In nuclear and hadronic physics, the Nakanishi PTIR has been applied to a wide range of physical problems, but so far restricted to the boundstate case, as shown, e.g., in Refs. [9, 11 13, 15 20]. The relativistic scattering states play a relevant role in many hadronic processes, like in the light-meson decays of heavy hadron resonances (see, e.g., for a recent study [21]), or even in more exotic cases (see e.g. [22]). Therefore, the extension of the Nakanishi PTIR for solving the BSE in the continuum region, as one of the possible tools to deal with the 4D Minkowski structure without approximations, is appealing and deserves dedicated efforts.

Recently, Carbonell and Karmanov proposed a new approach for obtaining, within PTIR, solutions of the BSE with truncated kernel, for bound states of both bosonic [11, 12, 18] and fermionic [19, 20] two-body systems, interacting through the exchange of a massive scalar boson (Yukawa Model). As in the case of Ref. [13] (where only the bosonic case was investigated), they didn't make use of the Wick rotation, but, for the first time, they exploited the covariant formulation of the Light-front (LF) field theory [23], obtaining a compact form for the kernel of the BSE, in both ladder [11] and cross-ladder [12] approximations. Within such an approach, it is possible to quickly get rid of the difficulties related to the analytic behavior of the BS amplitude in the Minkowski space, focusing the numerical efforts on determining a proper Nakanishi weight function, that remarkably are real functions, as mentioned above. The equation that determines such a weight function is directly related to the 3D integral equation for the valence component of the interacting-system state. In turn, such a 3D equation is obtained by projecting onto the null-plane the full BSE, after expressing the BS amplitude in terms of the Nakanishi PTIR. One may wonder how the valence wave function be able to generate the whole physical complexity of the full BS amplitude. Indeed, within a non perturbative framework, it formally turns out [24 29] that by introducing the pro- 
jection onto the null-plane, namely the analytic integration over the variable $k^{-}=k^{0}-k^{3}$, the full BS amplitude can be expressed in terms of the valence component and an operator, producing the richness of the Fock states on top of the lowest one. It must be emphasized that such a result has been obtained for both bound and scattering states of bosonic and fermionic systems. This gives us confidence that the PTIR approach, adopted for the bound state, could be extended to the scattering states.

Aim of this work is to construct the integral equation that determines the Nakanishi weight function for the scattering states of two massive scalar bosons, exchanging a massive scalar (cf [11] for the bound state). The main tool for obtaining the scattering equation is the projection onto the null-plane (LF-projection) of the BSE, as developed in a series of papers [24 28]. This method, not explicitly covariant, can be applied also to the bound-state case, obviously getting the same result like in Ref. [11], where the explicitly-covariant LF framework is considered. Moreover, the new integral equation is applied to the simple ladder approximation for the Yukawa model, and the extension of the Wick-Cutkosky model [3, 30] (a massless exchanged boson in ladder approximation) to the scattering region, is proposed.

The paper is organized as follows. In Sect. II, it is introduced both the general formalism of the BSE and the integral transform adopted for projecting both bound and scattering BSE's onto the LF hyper-plane (a short review on this topic can be found in Ref. [29]). In Sect. III, a new integral equation for determining the Nakanishi weight function for $4 \mathrm{D}$ scattering states is found, after briefly reviewing the bound state case. In Sect. IV] explicit expressions for both the inhomogeneous term and the kernel in ladder approximation are given for the scalar Yukawa model with an exchanged massive boson. Within such a framework, some relevant cases are discussed in Sect. $\nabla$, i) in subsect. $\mathrm{VA}$, the zero energy scattering amplitude, ii) in subsect. $\mathrm{VB}$, the Wick-Cutkosky model for scattering states and iii) in subsect. $\mathrm{VC}$, a new form for the integral equation determining the Nakanishi amplitude for bound states, in ladder approximation. Sect. VI contains concluding remarks. In Appendices $\mathrm{A}$ and $\mathrm{B}$ more details on the formal developments of Sect. IV are given.

\section{GENERAL FORMALISM AND LF PROJECTION OF THE BSE}

The ingredients of our approach are in order: i) the PTIR of the BS amplitude, $\Phi(k, p)$, and ii) the LF projection of the BSE introduced for obtaining a 3D equation for the valence 
wave function, $\psi\left(\xi, \mathbf{k}_{\perp}\right)$, for both bound and scattering states. Once the 3D equation is obtained, one can determine the Nakanishi amplitude, that in turn it yields the 4D BS amplitude within the PTIR [10]. It should be emphasized that the LF projection of the BSE in Minkowski space represents a smart way to deal with the singularities of the 4D $\Phi(k, p)$ once its analytic structure is known.

The inhomogeneous BSE for a scattering state of two scalar particles with equal mass $m$, total momentum $p=p_{1}+p_{2}$, total mass $M=\sqrt{p^{2}}$ and relative momentum $k=\left(p_{1}-p_{2}\right) / 2$ reads as follows

$$
\Phi^{(+)}\left(k, p, k_{i}\right)=(2 \pi)^{4} \delta^{(4)}\left(k-k_{i}\right)+G_{0}^{(12)}(k, p) \int \frac{d^{4} k^{\prime}}{(2 \pi)^{4}} i \mathcal{K}\left(k, k^{\prime}, p\right) \Phi^{(+)}\left(k^{\prime}, p, k_{i}\right),
$$

where $\Phi^{(+)}$is the BS amplitude for the scattering state, $i \mathcal{K}$ the interaction kernel, and $k_{i}$ the relative incoming momentum. It is worth noting that the matrix elements of the T-matrix involved in the scattering process are the half-off-shell ones, since the incoming particles are on their mass shell. This implies that the square of the intrinsic four-momentum is given by $k_{i}^{2}=m^{2}-M^{2} / 4 \leq 0$ (cf Sect. III). The normalization of the free states is

$$
\langle k \mid q\rangle=(2 \pi)^{4} \delta^{(4)}(k-q)
$$

and the free propagator of the two constituent particles is given by

$$
G_{0}^{(12)}(k, p)=G_{0}^{(1)} G_{0}^{(2)}=\frac{i}{\left(\frac{p}{2}+k\right)^{2}-m^{2}+i \epsilon} \quad \frac{i}{\left(\frac{p}{2}-k\right)^{2}-m^{2}+i \epsilon} .
$$

As well known, for a bound state one has the following homogeneous integral equation

$$
\Phi_{b}(k, p)=G_{0}^{(12)}(k, p) \int \frac{d^{4} k^{\prime}}{(2 \pi)^{4}} i \mathcal{K}\left(k, k^{\prime}, p\right) \Phi_{b}\left(k^{\prime}, p\right),
$$

The above equations represent the starting point of our investigation.

In the rest of the Section, the integral transform adopted for projecting both bound and scattering BSE's onto the LF hyper-plane is described. A reader already acquainted of both the Bethe-Salpeter formalism and the LF projection method can move directly to Section III.

\section{A. The BS amplitude and the LF wave-functions}

As well known, the BS amplitude is defined as the matrix element between the vacuum $\langle 0|$ and a state $|p\rangle$, with total momentum $p^{\mu}$, of the time ordered product of two Heisenberg 
operators. For a two-boson system, one has

$$
\Phi\left(x_{1}^{\mu}, x_{2}^{\mu}, p^{\mu}\right)=\left\langle 0\left|T\left\{\varphi_{H}\left(x_{1}^{\mu}\right) \varphi_{H}\left(x_{2}^{\mu}\right)\right\}\right| p\right\rangle .
$$

In general, the state vector $|p\rangle$ can be taken in different representations, but within the LF quantization with only massive quanta, it can be written in terms of an infinite sum over Fock components (see, e.g. 31]), i.e. the Fock vacuum is an exact eigenstate of the full LF Hamiltonian (see also [32] for a recent discussion of the simplicity of the LF vacuum). The weights of the Fock states in the infinite sum are the LF wave functions (LFWFs), $\psi_{n / p}$, that have a probabilistic interpretation, namely the proper integral over $\left|\psi_{n / p}\right|^{2}$ yields the probability of the corresponding Fock state. It should be pointed out that the LFWFs provide an intrinsic representation of the composite system, as a consequence of the possibility to separate the global motion from the intrinsic one, since the LF boosts belong to the kinematical subgroup, and the mass operator is a Lorentz scalar.

In terms of the four-momentum operator $\widehat{p}=\left(\widehat{p}^{+}, \widehat{p}^{-}, \widehat{\mathbf{p}}_{\perp}\right)$, with $\widehat{p}^{ \pm}=\widehat{p}^{0} \pm \widehat{p}^{3}$, the invariant square mass operator reads (see e.g.[31])

$$
\widehat{M}^{2}=\widehat{p}^{-} \widehat{p}^{+}-\widehat{\mathbf{p}}_{\perp}^{2}
$$

The eigenvalue problem to be solved is

$$
\widehat{M}^{2}|p\rangle=\mathcal{M}^{2}|p\rangle
$$

where $\mathcal{M}^{2}$ is the eigenvalue. The eigenstate, $|p\rangle$, can be expanded in multiparticle Fock eigenstates $\{|n\rangle\}$ of the free LF Hamiltonian, i.e a basis constructed by using the LF plane waves, $|\tilde{q}\rangle$. The single particle free state describes the motion of a particle with mass $m$ and it is an eigenstate of the LF momentum operators $\widehat{p}^{+}$and $\widehat{\mathbf{p}}_{\perp}$, i.e. $\tilde{q} \equiv\left\{q^{+}, \mathbf{q}_{\perp}\right\}$. The dependence upon the minus component, given by $q^{-}=\left(m^{2}+\left|\mathbf{q}_{\perp}\right|^{2}\right) / q^{+}$, is understood in the notation we have adopted. The free-state normalization is

$$
\langle\tilde{k} \mid \tilde{q}\rangle=2 k^{+}(2 \pi)^{3} \delta\left(k^{+}-q^{+}\right) \delta^{(2)}\left(\mathbf{k}_{\perp}-\mathbf{q}_{\perp}\right)=2 k^{+}(2 \pi)^{3} \delta^{3}(\tilde{k}-\tilde{q})
$$

with the completeness given by

$$
\int \frac{d^{3} \tilde{q}}{2 q^{+}(2 \pi)^{3}}|\tilde{q}\rangle\langle\tilde{q}|
$$


One should also recall that for a particle with mass $m$, one has

$$
\begin{aligned}
& \int d^{4} q \delta\left(q^{2}-m^{2}\right) \theta\left(q^{0}\right)=\frac{1}{2} \int d^{3} \tilde{q} \int d q^{-} \delta\left(q^{+} q^{-}-\left|\mathbf{q}_{\perp}\right|^{2}-m^{2}\right) \theta\left(q^{+}\right)= \\
& =\int \frac{d^{3} \tilde{q}}{2 q^{+}} \theta\left(q^{+}\right)
\end{aligned}
$$

where the positivity of $q^{+}$follows from the positivity of $q^{0}$, since the delta imposes that $q^{0}=\left[q^{+}+\left(m^{2}+\left|\mathbf{q}_{\perp}\right|^{2}\right) / q^{+}\right] / 2$.

Actually, each state $|p\rangle$ of the composite system is expanded in a complete Fock basis of non interacting $n$-particle states $|n\rangle$ with $n \geq 2$, since we are dealing with the BS amplitude of a composite two-boson system. Then one has

$$
|p\rangle=2(2 \pi)^{3} \sum_{n \geq 2}^{\infty} \int\left[d \xi_{i}\right]\left[d^{2} \mathbf{k}_{i \perp}\right] \psi_{n / p}\left(\xi_{i}, \mathbf{k}_{i \perp}\right)\left|n ; \xi_{i} p^{+}, \mathbf{k}_{i \perp}+\xi_{i} \mathbf{p}_{\perp}\right\rangle,
$$

where the properly symmetrized free state with $n$ particles are normalized, $\xi_{i}=q_{i}^{+} / p^{+}$and $\mathbf{q}_{i \perp}=\mathbf{k}_{i \perp}+\xi_{i} \mathbf{p}_{\perp}$, with $\mathbf{k}_{i \perp}$ the intrinsic transverse momentum of the $i-t h$ constituent, $\psi_{n / p}\left(\xi_{i}, \mathbf{k}_{i \perp}\right)$ are the LFWFs (see below). It should be pointed out that $\left\{\xi_{i}, \mathbf{k}_{i \perp}\right\}$ is the set of intrinsic variables, fulfilling the following relations

$$
\sum_{i=1}^{n} \xi_{i}=1 \quad \sum_{i=1}^{n} \mathbf{k}_{i \perp}=0
$$

In Eq. (11), the phase-space factors are given by

$$
\begin{gathered}
\int\left[d \xi_{i}\right] \equiv \prod_{i=1}^{n} \int \frac{d \xi_{i}}{2(2 \pi) \xi_{i}} \delta\left(1-\sum_{j=1}^{n} \xi_{j}\right), \\
\int\left[d^{2} \mathbf{k}_{i \perp}\right] \equiv \prod_{i=1}^{n} \int \frac{d^{2} \mathbf{k}_{i \perp}}{(2 \pi)^{2}} \delta^{2}\left(\sum_{j=1}^{n} \mathbf{k}_{j \perp}\right) .
\end{gathered}
$$

Moreover, the kinematical nature of the LF boosts allows one to write the overlap $\left\langle n ; \xi_{i} p^{+}, \mathbf{k}_{i \perp} \xi_{i} \mathbf{p}_{\perp} \mid p\right\rangle$ in terms of the global motion and an intrinsic function, i.e. the corresponding LFWF, $\psi_{n / p}\left(\xi_{i}, \mathbf{k}_{i \perp}\right)$, viz

$$
\begin{aligned}
& \left\langle n ; \xi_{i} p^{+}, \mathbf{k}_{i \perp}+\xi_{i} \mathbf{p}_{\perp} \mid p\right\rangle=2 p^{+}(2 \pi)^{3} \delta^{3}\left(\sum_{i=1}^{n} \tilde{q}_{i}-\tilde{p}\right) \psi_{n / p}\left(\xi_{i}, \mathbf{k}_{i \perp}\right)= \\
& =2(2 \pi)^{3} \delta\left(1-\sum_{i=1}^{n} \xi_{i}\right) \delta^{(2)}\left(\sum_{i=1}^{n} \mathbf{k}_{i \perp}\right) \psi_{n / p}\left(\xi_{i}, \mathbf{k}_{i \perp}\right)
\end{aligned}
$$

For a bound system, the LFWFs are normalized according to

$$
\sum_{n} \int\left[d \xi_{i}\right]\left[d^{2} \mathbf{k}_{i \perp}\right]\left|\psi_{n / p}\left(\xi_{i}, \mathbf{k}_{i \perp}\right)\right|^{2}=1
$$


Thus, given the Fock projection (14), the state of a composite system can be determined by reaching through a suitable $\mathrm{LF}$ boost the intrinsic frame, where $\mathbf{p}_{\perp}=0$, and then solving the eigenvalue problem for the invariant mass operator $\widehat{M}^{2}$, in order to obtain the set of LFWFs. It should pointed out that if one applies a dynamical LF rotation to the composite-system state, but still maintaining the Fock basis relative to the old orientation of the hyperplane, then the Fock content of the state changes. The content returns to be the same as before applying a dynamical rotation, if one adopts the Fock basis corresponding to the new orientation of the hyperplane. This is analogous to the Wigner transformations of the angular states, but generalized to the Fock space.

Summarizing, from Eqs. (5) and (11), one recognizes that all the Fock components with $n \geq 2$ are required for reconstructing the BS amplitude in Minkowski-space. This is easily understood, since the mass operator (6) is non-diagonal in the Fock space, due to the non commutativity of the interaction with respect to the particle-number operator. However, only the valence component $(n=2)$ is active in the calculation of the BS amplitude, (5), if we restrict the analysis to the equal LF-times case $\left(x_{1}^{+}=x_{2}^{+}\right)$(see below for details).

\section{B. The BS amplitude and its Fock content}

The general picture deduced from Eqs. (5) and (11), can be made more complete, if we take into account the results obtained in Refs. [24-28] for bound and scattering states of both two-boson and two-fermion systems. There, by using the LF projection technique (see also [29]), it was shown that the valence component of the LF bound and scattering states contains enough information for reconstructing the corresponding BS amplitudes. It should be pointed out that a similar result for the bound state of both two-boson and two-fermion systems, has been obtained in Refs. [11, 12, 19], but by applying the Nakanishi PTIR to the BS amplitude.

In what follows, the above mentioned relation between the valence component and the BS amplitude is briefly illustrated. From the field theoretical point of view, the states of the Fock basis, $|n\rangle$, are constructed by applying free-field creation operators to the vacuum state $|0\rangle$. Indeed, for a massive particle within the LF framework (let us recall that the quantization rules for the LF field theory [31] are given at fixed LF time $\tau=t+z / c$ [33]), the eigenvalue of $\widehat{p}^{+}$must be at least $m$, since the eigenvalues of $\widehat{p}^{2}$ and $\widehat{p}^{0}$ are both positive (the mass of 
the particle yields the lower bound). Then, the vacuum, that has a vanishing eigenvalue of $\widehat{p}^{+}$, cannot contain massive particles, given the conservation of the longitudinal momentum. This simple kinematical chain makes the Fock expansion meaningful (for a new vistas on the condensate in QCD see Ref. [32]). It should be pointed that the presence of the so-called zero-modes, that can be associated to particles with vanishing masses, spoils the triviality of the $\mathrm{LF}$ vacuum, i.e. $\widehat{p}^{+}|0\rangle=0$ does not more entail a vanishing number of particles. This means that the eigenvalue of $\widehat{p}^{+}$is not sufficient for identifying the vacuum with respect to a degenerate state populated by zero modes. In this Section, we are considering a massive theory.

In the composite system we are considering, the fundamental constituents appear in LF quantization as the excitations of the dynamical fields, $\varphi(x)$, expanded in terms of bosonic creation and annihilation operators on the null-plane, with coordinates $x^{-}=x^{0}-x^{3}$ and $\mathbf{x}_{\perp}$, at fixed LF time $x^{+}=x^{0}+x^{3}$ [31]. For $x^{+}=0$, the Heisenberg operators turn into the Schrödinger one, and therefore the field can be written in terms of non interacting creation and annihilation operators as follows (notice that $\varphi_{H}(x)=$ $\left.\exp \left(i P^{-} x^{+} / 2\right) \varphi(\tilde{x}) \exp \left(-i P^{-} x^{+} / 2\right)\right)$

$$
\varphi(\tilde{x})=\int \frac{d k^{+}}{\sqrt{2 k^{+}}} \frac{d^{2} \mathbf{k}_{\perp}}{(2 \pi)^{3 / 2}} \theta\left(k^{+}\right)\left(a^{\dagger}(\tilde{k}) e^{-i \tilde{k} \cdot \tilde{x}}+a(\tilde{k}) e^{i \tilde{k} \cdot \tilde{x}}\right),
$$

where $\tilde{x}=\left(x^{-}, \mathbf{x}_{\perp}\right)$ and $\tilde{x} \cdot \tilde{k}=k^{+} x^{-} / 2-\mathbf{x}_{\perp} \cdot \mathbf{k}_{\perp}$ (in this notation $x^{+}=0$ is dropped off). The creation and annihilation operators satisfy the following commutation relations

$$
\left[a(\tilde{k}), a^{\dagger}\left(\tilde{k}^{\prime}\right)\right]=\delta\left(k^{+}-k^{\prime+}\right) \delta^{(2)}\left(\mathbf{k}_{\perp}-\mathbf{k}_{\perp}^{\prime}\right) .
$$

A one-particle free state is defined by $|\tilde{k}\rangle=(2 \pi)^{3 / 2} \sqrt{2 k^{+}} a^{\dagger}(\tilde{k})|0\rangle$, given the adopted normalization ( $\operatorname{cf}$ Eq. (8) ), and $\langle\tilde{x} \mid \tilde{k}\rangle=e^{i \tilde{k} \cdot \tilde{x}}$.

Let us express $\Phi\left(x_{1}, x_{2}, p\right)$ in (5) through its Fourier transform. Translational invariance imposes to $\Phi$ the following form

$$
\Phi\left(x_{1}, x_{2}, p\right)=\tilde{\Phi}(x, p) e^{-i p \cdot X},
$$

where $X^{\mu}=\left(x_{1}^{\mu}+x_{2}^{\mu}\right) / 2, x^{\mu}=x_{1}^{\mu}-x_{2}^{\mu}$ and $\tilde{\Phi}(x, p)$ the reduced amplitude. It is related to its Fourier transform, $\Phi(k, p)$, as follows

$$
\tilde{\Phi}(x, p)=\int \frac{d^{4} k}{(2 \pi)^{4}} e^{i k \cdot x} \Phi(k, p)
$$


where

$$
p^{\mu}=p_{1}^{\mu}+p_{2}^{\mu} \quad k^{\mu}=\frac{p_{1}^{\mu}-p_{2}^{\mu}}{2}
$$

with $p_{i}^{2} \neq m^{2}$ (see Eq. (10)). The amplitude $\Phi(k, p)$ satisfies the homogeneous, (4), or the inhomogeneous, (11), BS equation depending if one is considering bound or scattering states.

For $X^{\mu}=0$, one has

$$
\begin{aligned}
& \tilde{\Phi}(x, p)=\left\langle 0\left|T\left\{\varphi_{H}\left(x^{\mu} / 2\right) \varphi_{H}\left(-x^{\mu} / 2\right)\right\}\right| p\right\rangle= \\
& =\theta\left(x^{+}\right)\left\langle 0\left|\varphi(\tilde{x} / 2) e^{-i P^{-} x^{+} / 2} \varphi(-\tilde{x} / 2)\right| p\right\rangle e^{i p^{-} x^{+} / 4}+ \\
& +\theta\left(-x^{+}\right)\left\langle 0\left|\varphi(-\tilde{x} / 2) e^{i P^{-} x^{+} / 2} \varphi(\tilde{x} / 2)\right| p\right\rangle e^{-i p^{-} x^{+} / 4}= \\
& =\theta\left(x^{+}\right) \sum_{n, n^{\prime}} e^{i p^{-} x^{+} / 4}\left\langle 0|\varphi(\tilde{x} / 2)| n^{\prime}\right\rangle\left\langle n^{\prime}\left|e^{-i P^{-} x^{+} / 2}\right| n\right\rangle\langle n|\varphi(-\tilde{x} / 2)| p\rangle+ \\
& +\theta\left(-x^{+}\right) \sum_{n, n^{\prime}} e^{-i p^{-} x^{+} / 4}\left\langle 0|\varphi(-\tilde{x} / 2)| n^{\prime}\right\rangle\left\langle n^{\prime}\left|e^{i P^{-} x^{+} / 2}\right| n\right\rangle\langle n|\varphi(\tilde{x} / 2)| p\rangle
\end{aligned}
$$

where $|n\rangle$ and $\left|n^{\prime}\right\rangle$ are states of the Fock basis used in Eq. (11). It is easily realized that the matrix elements $\left\langle n^{\prime}\left|e^{-i P^{-} x^{+} / 2}\right| n\right\rangle$ are not diagonal in the Fock space, apart the case $x^{+}=0$, since the operator $P^{-}$contains the interaction in its full glory. Therefore, all the Fock components are acting in BS amplitude of the composite system.

\section{The BS amplitude and the valence wave-function}

The interesting case $x^{+}=0$ leads straightforwardly to the relation between the valence component, $\psi_{n=2 / p}$, and the BS amplitude (see also [23]). In particular, one has

$$
\lim _{x^{+} \rightarrow 0_{+}} \tilde{\Phi}(x, p)=\langle 0|\varphi(\tilde{x} / 2) \varphi(-\tilde{x} / 2)| p\rangle
$$

If we perform the $4 \mathrm{D}$ Fourier transform of the quantity $\tilde{\Phi}(x, p) \delta\left(x^{+}\right)$(i.e restricting the reduced amplitude to the LF hypersurface, or equal LF-times case $\left(x^{+}=x_{1}^{+}-x_{2}^{+}=0\right)$ ), one can extract the valence wave function $\psi_{n=2 / p}\left(\xi, \mathbf{k}_{\perp}\right)$. As a matter of fact, on one hand, one can write

$$
\begin{aligned}
& \int d^{3} \tilde{x} e^{-i \tilde{q} \cdot \tilde{x}} \tilde{\Phi}\left(\tilde{x}, x^{+}=0, p\right)=\frac{1}{2} \int \frac{d k^{-}}{2 \pi} \int \frac{d^{3} \tilde{k}}{(2 \pi)^{3}} \Phi(k, p) \int d^{3} \tilde{x} e^{i(\tilde{k}-\tilde{q}) \cdot \tilde{x}}= \\
& =\int_{-\infty}^{\infty} \frac{d k^{-}}{2 \pi} \Phi(k, p) .
\end{aligned}
$$


On the other hand, one has

$$
\begin{aligned}
& \int d^{3} \tilde{x} e^{-i \tilde{k} \cdot \tilde{x}} \tilde{\Phi}\left(\tilde{x}, x^{+}=0_{+}, p\right)=\int d^{3} \tilde{x} e^{-i \tilde{k} \cdot \tilde{x}}\langle 0|\varphi(\tilde{x} / 2) \varphi(-\tilde{x} / 2)| p\rangle= \\
& =\int \frac{d^{3} \tilde{p}_{1}}{\sqrt{2 p_{1}^{+}}} \int \frac{d^{3} \tilde{p}_{2}}{\sqrt{2 p_{2}^{+}}} 2 \delta^{3}\left(\tilde{k}-\frac{\tilde{p}_{1}-\tilde{p}_{2}}{2}\right) \int \frac{d^{3} \tilde{q}_{1}}{2 q_{1}^{+}(2 \pi)^{3}} \int \frac{d^{3} \tilde{q}_{2}}{2 q_{2}^{+}(2 \pi)^{3}} 2 p^{+}(2 \pi)^{3} \times \\
& \delta^{3}\left(\tilde{p}-\tilde{q}_{1}-\tilde{q}_{2}\right) \frac{1}{\sqrt{2}} \psi_{n=2 / p}\left(\xi, \mathbf{k}_{\perp}\right) \sqrt{2 q_{1}^{+}} \sqrt{2 q_{2}^{+}}(2 \pi)^{3}\left\langle 0\left|a\left(\tilde{p}_{1}\right) a\left(\tilde{p}_{2}\right) a^{\dagger}\left(\tilde{q}_{1}\right) a^{\dagger}\left(\tilde{q}_{2}\right)\right| 0\right\rangle= \\
& =\frac{p^{+} \sqrt{2}}{\left(\frac{p}{2}+k\right)^{+}\left(\frac{p}{2}-k\right)^{+}} \psi_{n=2 / p}\left(\xi, \mathbf{k}_{\perp}\right)
\end{aligned}
$$

Summarizing, the valence component is given by the Fourier transform of $\tilde{\Phi}\left(\tilde{x}, x^{+}=0_{+}, p\right)$, and it reads (cf [23])

$$
\psi_{n=2 / p}\left(\xi, \mathbf{k}_{\perp}\right)=\frac{p^{+}}{\sqrt{2}} \xi(1-\xi) \int_{-\infty}^{\infty} \frac{d k^{-}}{2 \pi} \Phi(k, p)=\frac{p^{+}}{\sqrt{2}} \xi(1-\xi) \phi_{L F}\left(\xi, \mathbf{k}_{\perp}\right)
$$

where $\xi=\xi_{1}$ and we have introduced the notation $\phi_{L F}\left(\xi, \mathbf{k}_{\perp}\right)$ for future purpose (see the next subsection). The expression (25) can be considered as a first bridge between the Fock state decomposition of a composite-system state, Eq. (11), and the BS amplitude. In particular, the integration over $k^{-}$projects the BS amplitude $\Phi(k, p)$ onto the LF-hyperplane, i.e. $x^{+}=0$, and it leads to the valence wave-function.

The LF projection can be directly applied to both the homogeneous and inhomogeneous BSE (see Refs. [11, 12] for the LF covariant approach for the bound state case). For instance, from Eq. (41) one has for the bound state

$$
\int \frac{d k^{-}}{2 \pi} \Phi_{b}(k, p)=\int \frac{d k^{-}}{2 \pi} G_{0}^{(12)}(k, p) \int \frac{d^{4} k^{\prime}}{(2 \pi)^{4}} i \mathcal{K}\left(k, k^{\prime}, p\right) \Phi_{b}\left(k^{\prime}, p\right),
$$

This mathematical step is a fundamental one in order to get an equivalent equation that allows one to determine the Nakanishi amplitude, eventually leading to the BS equation treated within PTIR framework[10], (as discussed in detail in the next Section). In what follows, the LF projection is applied to both bound and scattering valence wave-functions.

\section{The LF projection of the BS equation}

The LF projection, applied to the BS amplitudes for bosonic and fermionic states, can be embedded in a more general framework [24 28], where the relation (25) can be extended to the scattering BSE without relying on any perturbative approach: we reiterate that this 
represents the main motivation of the present paper. It should be also mentioned that the general formalism of Refs. [24 28] allows one to fully reconstruct the 4D BS amplitude in Minkowski space from the 3D valence component (cf the following Eqs. (39) and (40)).

Let us briefly review the results for an interacting two-boson system [24, 26, 27] (see Refs. [25), 28] for the generalization to a fermionic system and Ref. [29] for an introduction to the three-boson case). In order to get the $3 \mathrm{D}$ eigenfunction for the valence component from the 4D BSE, the LF projection technique is combined with a Quasi-potential treatment of the BS interacting kernel (see [34]). This new ingredient makes it possible to single out from the 4D Green's function of the interacting system the "trivial" global propagation. Such a formal step is accomplished by using the LF projection, i.e. the proper integration over the $k^{-}$variable. Then, one remains with the valence dynamics described by a $3 \mathrm{D}$ effective LF interaction directly related to the BS kernel, through suitable projection operators.

The main outcome for bound and scattering states is given by the 3D LF eigenequation for the valence component that can be obtained by applying the LF-projection technique to the BS equation. This can be rewritten as follows

$$
G^{-1}(p)|\Psi\rangle=\left[G_{0}^{-1}(p)-V(p)\right]|\Psi\rangle=0
$$

where an overall delta function for the total four-momentum conservation has been factorized out, $G_{0}(p)$ is the free propagator and $V(p)$ is the $4 \mathrm{D}$ interaction, obtained from some given Lagrangian [35]. Their matrix elements are given by

$$
\begin{aligned}
& \left\langle p_{1}\left|G_{0}(p)\right| p_{1}^{\prime}\right\rangle=\delta^{4}\left(p_{1}^{\prime}-p_{1}\right) G_{0}^{(12)}(k, p) \\
& \left\langle p_{1}|V(p)| p_{1}^{\prime}\right\rangle=i \mathcal{K}\left(k, k^{\prime}, p\right)
\end{aligned}
$$

where $p=p_{1}+p_{2}=p_{1}^{\prime}+p_{2}^{\prime}, k=\left(p_{1}-p_{2}\right) / 2$ and $k^{\prime}=\left(p_{1}^{\prime}-p_{2}^{\prime}\right) / 2$. Then, following Refs. [24 28] and the resumé in Ref. [29], one obtains the following 3D LF eigenequation (that holds for bound and scattering states, depending upon the boundary conditions)

$$
\left\langle k^{+}, \mathbf{k}_{\perp}\left|g_{0}^{-1}(p)-w(p)\right| \psi_{n=2 / p}\right\rangle=0
$$

where the 3D free Green's function, $g_{0}(p)$ describes the global propagation and $w(p)$ is the effective 3D interaction, related to the 4D Quasi-potential (cf [34]).

$$
W(p)=V(p)+V(p) \Delta_{0}(p) W(p)
$$


where the key ingredients is contained in $\Delta_{0}(p)=G_{0}(p)-\tilde{G}_{0}(p)$ and it is represented by the auxiliary Green's function $\tilde{G}_{0}(p)$, that is nothing else but the 4D image of the 3D $g_{0}(p)$. Through $\Delta_{0}(p)$, one can factorize the intrinsic free motion, after subtracting the "trivial" global one. The 3D free Green's function is defined by

$$
g_{0}(p)=\ell \downarrow G_{0}(p) \downarrow_{r}
$$

where the two new symbols $\ell \downarrow$ and $\downarrow_{r}$, that indicate a projection onto the LF hyperplane $x^{+}=0$, are given by

$$
\ell \downarrow \equiv \int \frac{d k_{1}^{-}}{2 \pi}\left\langle k_{1}^{-}\left|, \quad \downarrow_{r} \equiv \int \frac{d k_{1}^{-}}{2 \pi}\right| k_{1}^{-}\right\rangle
$$

Then, 3D quantities in Eq. (29) have the following expressions

$$
\begin{aligned}
& g_{0}(p)^{-1}=\bar{\Pi}_{0}(p)\left[G_{0}(p)\right]^{-1} \Pi_{0}(p) \\
& w(p):=\bar{\Pi}_{0}(p) W(p) \Pi_{0}(p)
\end{aligned}
$$

where the free reverse LF projection operator and its LF-conjugated, are

$$
\Pi_{0}(p):=G_{0}(p) \downarrow_{r} \quad g_{0}(p)^{-1}, \quad \quad \bar{\Pi}_{0}(p):=g_{0}(p)^{-1} \ell \downarrow G_{0}(p) .
$$

Finally, the 4D auxiliary Green's function reads

$$
\tilde{G}_{0}(p)=\Pi_{0}(p) g_{0}(p) \bar{\Pi}_{0}(p)
$$

It has to point out that $\Pi_{0}(p)$ action is $3 D \rightarrow 4 D$, while $\bar{\Pi}_{0}(p)$ acts in the reverse way. The explicit expression of $g_{0}^{-1}(p)$ is

$$
g_{0}^{-1}(p)=-i \theta\left(\widehat{k}_{1}^{+}\right) \theta\left(p^{+}-\widehat{k}_{1}^{+}\right) \widehat{k}_{1}^{+}\left(p^{+}-\widehat{k}_{1}^{+}\right)\left[p^{-}-\widehat{k}_{1 o n}^{-}-\widehat{k}_{2 o n}^{-}+i \epsilon\right] .
$$

where

$$
\widehat{k}_{1 \text { on }}^{-}\left|\tilde{k}_{1}\right\rangle=\frac{m^{2}+\left|\mathbf{k}_{1 \perp}\right|^{2}}{k_{1}^{+}}\left|\tilde{k}_{1}\right\rangle
$$

and the analogous for particle 2. The diagrammatic content (in the Fock space) of the Quasi-potential can be argued from the following formal solution of the integral equation that determines $W(p)$

$$
W(p)=V(p)+V(p) \Delta_{0}(p) W(p)=V(p) \sum_{N=0}^{\infty}\left[\Delta_{0}(p) V(p)\right]^{N}
$$


where the $\Delta_{0}(p)$ insertions bring information on the intermediate particle propagations. A corresponding analysis can be performed for the LF effective interaction $w(p)$, and it is physically quite transparent, since it can be carried out within a LF-time ordered framework. In particular, $w(p)$ contains all possible LF-time ordered exchanges, corresponding to a sum over diagrams with any number of intermediate particles: this happens even for the ladder BSE. It should be pointed out that the ladder approximation of the LF eigenequation, Eq. (29), where $w(p)$ is approximated by the first term in the power expansion in Eq. (38), does not account for the full complexity of the 4D ladder BSE. The physical reason lies in the fact that the iterations of the 4D ladder kernel and the 3D LF ladder kernel (time-ordered graphs) generate different intermediate states. The LF ladder kernel and its iterations contain only one exchanged particle (at a given global LF-time ) in the intermediate state, whereas the iterations of the ladder 4D kernel contain also many-body states, with increasing number of exchanged particles (stretched boxes, see e.g. [36]). This leads to a difference in the binding energies, which is however small [24, 37, 38]. In principle, for any 4D kernel given by a finite set of irreducible graphs, both BS (27) and LF equation, Eq. (29), gives the same eigenvalue, once $w(p)$ comes from the solution of (38). [28])

In the Quasi-potential framework the interacting LF reverse projection operator (see Ref.

$$
\Pi(p)=\left[1+\Delta_{0}(p) W(p)\right] \Pi_{0}(p)
$$

leads to the following relations between the $3 \mathrm{D}$ valence component and the 4D BS amplitude

$$
\begin{aligned}
& |\Psi\rangle=\Pi(p)\left|\phi_{L F}\right\rangle \\
& \ell \downarrow|\Psi\rangle=\left|\phi_{L F}\right\rangle .
\end{aligned}
$$

The 3D valence wave-function, $\phi_{L F}$, has been already introduced in Eq. (25). The analogous relations for the free case read

$$
\begin{aligned}
& \left|\Psi_{0}\right\rangle=\Pi_{0}(p)\left|\psi_{0}\right\rangle \\
& \ell \downarrow\left|\Psi_{0}\right\rangle=\left|\psi_{0}\right\rangle .
\end{aligned}
$$

It turns out that the full complexity of the Fock-space in the 4D BS amplitude appears, not only through the effective interaction that determines the valence wave function, but also through the interacting reverse projection operator, $\Pi(p)$. 
Finally, let us remind the 3D integral equations that follow from the eigenequation (29), for both the bound and the scattering states, viz

$$
\begin{aligned}
& \phi_{L F}^{b}\left(\xi, \mathbf{k}_{\perp}\right)=\left\langle k^{+}, \mathbf{k}_{\perp}\left|g_{0}(p) w(p)\right| \phi_{L F}^{b}\right\rangle, \\
& \phi_{L F}^{(+)}\left(\xi, \mathbf{k}_{\perp}\right)=\left\langle k^{+}, \mathbf{k}_{\perp}|1+g(p) w(p)| \psi_{0}\right\rangle,
\end{aligned}
$$

with $g_{0}^{-1}(p)\left|\psi_{0}\right\rangle=0$.

\section{INTEGRAL EQUATION FOR THE NAKANISHI WEIGHT FUNCTION FOR BOUND AND SCATTERING STATES}

In this Section, we illustrate how to obtain an integral equation for the Nakanishi weight functions for scattering states, starting from a BS equation for a system composed by two massive scalars, exchanging a scalar particle. The kernel is composed by the infinite sum of

two-particle irreducible diagrams [35, 39], and the self-energy contribution to the massive two-particle propagation are discarded, at the present stage.

In order to have a suitable introduction to the scattering case, we first briefly discuss the S-wave bound-state case, within our LF approach. The same integral equation for the Nakanishi weight function has been devised by Carbonell and Karmanov [11, 12], but within the explicitly covariant LF approach [23].

\section{A. The bound states}

In this subsection the S-wave bound-state integral equation for determining the Nakanishi weight function is presented by using the LF language introduced in the previous Section (namely a non explicitly covariant formalism). The BS amplitude is written in terms of the Nakanishi PTIR [2, 10, 11], $g_{b}\left(\gamma^{\prime}, z^{\prime} ; \kappa^{2}\right)$, as follows

$$
\begin{aligned}
& \Phi_{b}(k, p)=-i \int_{-1}^{1} d z^{\prime} \int_{0}^{\infty} d \gamma^{\prime} \frac{g_{b}\left(\gamma^{\prime}, z^{\prime} ; \kappa^{2}\right)}{\left[\gamma^{\prime}+m^{2}-\frac{1}{4} p^{2}-k^{2}-p \cdot k z^{\prime}-i \epsilon\right]^{2+n}}= \\
& =i(-1)^{n} \int_{-1}^{1} d z^{\prime} \int_{0}^{\infty} d \gamma^{\prime} \frac{g_{b}\left(\gamma^{\prime}, z^{\prime} ; \kappa^{2}\right)}{\left[k^{2}+p \cdot k z^{\prime}-\gamma^{\prime}-\kappa^{2}+i \epsilon\right]^{2+n}}
\end{aligned}
$$

where $p^{2}=M^{2}$ is the invariant mass of the interacting system and

$$
\kappa^{2}=m^{2}-\frac{M^{2}}{4} .
$$


with $m$ the constituent mass. For bound states $\kappa^{2}>0$, while for the scattering ones $\kappa^{2} \leq 0$. In general, the power $n$ in the denominator is a dummy integer parameter, and in Refs. [11, 12] the minimal value $n=1$ was chosen for the sake of simplicity. Bigger values of $n$ may result in a more smooth solution for $g_{b}\left(\gamma^{\prime}, z^{\prime} ; \kappa^{2}\right)[13,20]$.

The LF projection allows one to readily get rid of the singularity in Eq. (45), eventually obtaining the valence wave function, within the PTIR framework. In agreement with Ref. [11], one can define $\psi_{b}=\sqrt{2} \psi_{n=2 / p}$ as follows (cf Eq. (25))

$$
\begin{aligned}
& \psi_{b}\left(\xi, k_{\perp}\right)=p^{+} \xi(1-\xi) \int \frac{d k^{-}}{2 \pi} \Phi_{b}(k, p)= \\
& =\xi(1-\xi) \int_{0}^{\infty} d \gamma^{\prime} \frac{g_{b}\left(\gamma^{\prime}, 1-2 \xi ; \kappa^{2}\right)}{\left[\gamma^{\prime}+k_{\perp}^{2}+\kappa^{2}+(2 \xi-1)^{2} \frac{M^{2}}{4}\right]^{2}} .
\end{aligned}
$$

It should be pointed out that the denominator is always positive, and one can safely drop out the $i \epsilon$ term.

In order to strictly follow the notation of Ref. [11], let us use the variables $(\gamma, z)$, related to the standard LF momentum as follows:

$$
\gamma=k_{\perp}^{2} \quad 1 \geq z=1-2 \xi \geq-1
$$

Then, one can rewrite

$$
\psi_{b}(z, \gamma)=\frac{\left(1-z^{2}\right)}{4} \int_{0}^{\infty} d \gamma^{\prime} \frac{g_{b}\left(\gamma^{\prime}, z ; \kappa^{2}\right)}{\left[\gamma^{\prime}+\gamma+z^{2} m^{2}+\left(1-z^{2}\right) \kappa^{2}\right]^{2}}
$$

The integral equation for the Nakanishi weight function, $g_{b}\left(\gamma, z ; \kappa^{2}\right)$, is obtained by inserting (45) in both sides of (26), and using Eqs. (47) and (49)(see Appendix A of [11]) for details). One gets

$$
\int_{0}^{\infty} d \gamma^{\prime} \frac{g_{b}\left(\gamma^{\prime}, z ; \kappa^{2}\right)}{\left[\gamma^{\prime}+\gamma+z^{2} m^{2}+\left(1-z^{2}\right) \kappa^{2}\right]^{2}}=\int_{0}^{\infty} d \gamma^{\prime} \int_{-1}^{1} d z^{\prime} V_{b}^{L F}\left(\gamma, z ; \gamma^{\prime}, z^{\prime}\right) g_{b}\left(\gamma^{\prime}, z^{\prime} ; \kappa^{2} \gamma 50\right)
$$

where the kernel $V_{b}$, appearing in the right-hand side of Eq. (50), is related to the kernel $i \mathcal{K}$ in the BS equation, (11), as follows

$$
\begin{aligned}
& V_{b}^{L F}\left(\gamma, z ; \gamma^{\prime}, z^{\prime}\right)=i p^{+} \int_{-\infty}^{\infty} \frac{d k^{-}}{2 \pi} G_{0}^{(12)}(k, p) \int \frac{d^{4} k^{\prime}}{(2 \pi)^{4}} \frac{i \mathcal{K}\left(k, k^{\prime}, p\right)}{\left[k^{\prime 2}+p \cdot k^{\prime} z^{\prime}-\gamma^{\prime}-\kappa^{2}+i \epsilon\right]^{3}}= \\
& =-i p^{+} \int_{-\infty}^{\infty} \frac{d k^{-}}{2 \pi} \frac{1}{\left[\left(\frac{p}{2}+k\right)^{2}-m^{2}+i \epsilon\right]} \frac{1}{\left[\left(\frac{p}{2}-k\right)^{2}-m^{2}+i \epsilon\right]} \times \\
& \int \frac{d^{4} k^{\prime}}{(2 \pi)^{4}} \frac{i \mathcal{K}\left(k, k^{\prime}, p\right)}{\left[k^{\prime 2}+p \cdot k^{\prime} z^{\prime}-\gamma^{\prime}-\kappa^{2}+i \epsilon\right]^{3}}
\end{aligned}
$$


Its explicit expression within both the ladder and the cross-ladder approximation for the kernel $\mathcal{K}$ can be found in Refs. [11, 12].

We emphasize that Eq. (50) is equivalent to the initial BS equation (1), within the PTIR framework. Moreover, it should be pointed out that the mass, $M$, of the interacting system appears on both sides of Eq. (50) and is contained in the parameter $\kappa^{2}$. Once the weight function $g_{b}\left(\gamma^{\prime}, z ; \kappa^{2}\right)$ is obtained, then we can reconstruct the BS amplitude (see Eq. (45)). As already mentioned in the Introduction, the ability of the valence wave function to reconstruct the full BS amplitude is not only restricted to a perturbative framework, but it holds also in a non perturbative analysis, and even more for both bound and scattering states [24 28]. This observation represented our motivation to face with the investigation of the scattering sates.

Finally some important remarks. It turns out [11, 13] that $g_{b}\left(\gamma, z ; \kappa^{2}\right)$ may be zero in an interval $0 \leq \gamma \leq \gamma_{0}$, and that the exact value where it differs from zero is determined by Eq. (50) itself. Moreover, the uniqueness of the Nakanishi weight function is ensured by a theorem (see [10]). Following Refs. [13, 14], one can extract from (50) the following eigenequation

$$
g_{b}\left(\gamma, z ; \kappa^{2}\right)=\int_{0}^{\infty} d \gamma^{\prime} \int_{-1}^{1} d z^{\prime} \mathcal{V}_{b}\left(\gamma, z ; \gamma^{\prime}, z^{\prime} ; \kappa^{2}\right) g_{b}\left(\gamma^{\prime}, z^{\prime} ; \kappa^{2}\right)
$$

For a discussion of the above equation within the ladder-approximation framework, see subsect. VC,

\section{B. The scattering states}

The LF-projection technique, combined with the PTIR framework, has the appealing feature of systematically removing singularities, that appear in both BS amplitude and kernel, by integrating over $k^{-}$more simple analytical structures. We notice that the quadratic $k^{0}$ dependence in the propagators translates into a linear dependence upon $k^{-}$(see, e.g. [40, 41]). Since within PTIR approach, the analytical form of any multi-leg amplitude is made explicit, the linearity of the pole structure attained through the LF technique is advantageous. Then, it is natural to extend the investigation to the scattering case, increasing the complexity of the problem.

According to the general treatment of a four-leg amplitude, as developed in Ref. [2, 10], 
one can write the off-shell T-matrix as follows

$$
\begin{gathered}
\left\langle k^{\prime \mu}|T(p)| k^{\mu}\right\rangle=\prod_{i=1,7} \int_{0}^{1} d z_{i} \delta\left(1-\sum_{j=1,7} z_{j}\right) \int_{0}^{\infty} d \gamma \times \\
\gamma-\left[z_{1}\left(p / 2+k^{\prime}\right)^{2}+z_{2}\left(p / 2-k^{\prime}\right)^{2}+z_{3}(p / 2+k)^{2}+z_{4}(p / 2-k)^{2}+z_{5} s+z_{6} t+z_{7} u\right]-i \epsilon
\end{gathered}
$$

where the Mandelstam variables are

$$
s=p^{2} \quad t=\left(k^{\prime}-k\right)^{2} \quad u=\left(k^{\prime}+k\right)^{2}
$$

and they satisfied the four-momentum conservation, that reads

$$
s+t+u=p^{2}+2\left(k^{2}+k^{2}\right)
$$

Equation (53) has a redundant dependence upon the seven invariant squares, in order to have a compact form of the fully-off-shell four-leg amplitude (namely, without a decomposition in $s, t$ and $u$ channels). It is understood that the actual dependence is upon a set of six independent variables, given the constraint in Eq. (55).

Indeed, for evaluating the scattering states we need half-off-shell T-matrix, i.e. one has to put on their-own mass shell the two incoming particles with four-momenta $p / 2 \pm k_{i}$. Then one has

$$
\begin{gathered}
\left\langle k^{\mu}|T(p)| k_{i}^{\mu}\right\rangle=\Pi_{i=1,7} \int_{0}^{1} d z_{i} \delta\left(1-\sum_{j=1,7} z_{j}\right) \int_{0}^{\infty} d \gamma \times \\
\gamma-\left[z_{1}(p / 2+k)^{2}+z_{2}(p / 2-k)^{2}+z_{3} m^{2}+z_{4} m^{2}+z_{5} p^{2}+z_{6} t+z_{7} u\right]-i \epsilon
\end{gathered}
$$

where $\left(p / 2+k_{i}\right)^{2}=\left(p / 2-k_{i}\right)^{2}=m^{2}$. As a consequence $p \cdot k_{i}=0$, and therefore $k_{i}^{2}=$ $m^{2}-p^{2} / 4=\kappa^{2} \leq 0$.

Let us analyze in detail the $z$-dependent part of the denominator in Eq. (56). By exploiting the presence of the delta function in Eq. (56) for eliminating $z_{5}$, it can be rewritten as follows

$$
\begin{aligned}
& z_{1}(p / 2+k)^{2}+z_{2}(p / 2-k)^{2}+\left(z_{3}+z_{4}\right) m^{2}+z_{5} p^{2}+z_{6} t+z_{7} u= \\
& =\left(2-2 z_{1}-2 z_{2}-2 z_{3}-2 z_{4}-3 z_{6}-3 z_{7}\right) \frac{p^{2}}{2}+\left(z_{1}+z_{2}+z_{3}+z_{4}+2 z_{6}+2 z_{7}\right) m^{2}+ \\
& +\left(z_{1}+z_{2}+z_{6}+z_{7}\right)\left[\left(k^{2}+\frac{p^{2}}{4}-m^{2}\right)+\zeta^{\prime} p \cdot k+\zeta 2 k \cdot k_{i}\right]
\end{aligned}
$$


where $\zeta=\left(z_{7}-z_{6}\right) /\left(z_{1}+z_{2}+z_{6}+z_{7}\right)$ and $\zeta^{\prime}=\left(z_{1}-z_{2}\right) /\left(z_{1}+z_{2}+z_{6}+z_{7}\right)$ belong to $[-1,1]$. Remind that the variables $z_{i}$ fulfill i) $\sum_{i=1,7} z_{i}=1$ and ii) $0 \leq z_{i} \leq 1$. Furthermore by introducing a new variable

$$
\gamma^{\prime \prime}=\frac{\gamma+\left(p^{2}-m^{2}\right)\left(z_{1}+z_{2}+z_{3}+z_{4}+2 z_{6}+2 z_{7}\right)}{\left(z_{1}+z_{2}+z_{6}+z_{7}\right)}-\frac{p^{2}}{2} \frac{\left(2-z_{6}-z_{7}\right)}{\left(z_{1}+z_{2}+z_{6}+z_{7}\right)}
$$

that belongs to $[-\infty, \infty]$, recalling that $\left(z_{1}+z_{2}+z_{6}+z_{7}\right) \in[0,1]$, then one can rewrite the half off-shell T-matrix as follows

$$
\left\langle k^{\mu}|T(p)| k_{i}^{\mu}\right\rangle=\int_{-1}^{1} d \zeta \int_{-1}^{1} d \zeta^{\prime} \int_{-\infty}^{\infty} d \gamma^{\prime \prime} \frac{\widetilde{\mathcal{G}}^{+}\left(\gamma^{\prime \prime}, \zeta, \zeta^{\prime}\right)}{\gamma^{\prime \prime}-\left[k^{2}+\frac{p^{2}}{4}-m^{2}+\zeta^{\prime} p \cdot k+\zeta 2 k \cdot k_{i}\right]-i \epsilon}
$$

where the weight function, $\widetilde{\mathcal{G}}^{+}\left(\gamma^{\prime \prime}, \zeta, \zeta^{\prime}\right)$, is the result of multifold integrations and changes of variables, as suggested by the previous calculation. The scattering wave can be obtained by considering the following vertex function

$$
\begin{aligned}
& \left\langle k^{\mu}\left|G_{0}(p) T(p)\right| k_{i}^{\mu}\right\rangle=\frac{i}{\left(\frac{p}{2}+k\right)^{2}-m^{2}+i \epsilon} \frac{i}{\left(\frac{p}{2}-k\right)^{2}-m^{2}+i \epsilon} \times \\
& \int_{-1}^{1} d \zeta \int_{-1}^{1} d \zeta^{\prime} \int_{-\infty}^{\infty} d \gamma^{\prime \prime} \frac{\widetilde{\mathcal{G}}^{+}\left(\gamma^{\prime \prime}, \zeta, \zeta^{\prime}\right)}{\left[k^{2}+\frac{p^{2}}{4}-m^{2}+\zeta^{\prime} p \cdot k+\zeta 2 k \cdot k_{i}\right]-\gamma^{\prime \prime}+i \epsilon}= \\
& =\int_{-1}^{1} d \zeta \int_{-1}^{1} d \zeta^{\prime} \int_{-\infty}^{\infty} d \gamma^{\prime \prime} \int_{0}^{1} d \alpha_{1} \int_{0}^{1-\alpha_{1}} d \alpha_{2} \frac{\widetilde{\mathcal{G}}^{+}\left(\gamma^{\prime \prime}, \zeta, \zeta^{\prime}\right)}{[D+i \epsilon]^{3}}
\end{aligned}
$$

where the Feynman trick

$$
\frac{1}{A B C}=\int_{0}^{1} d \alpha_{1} \int_{0}^{1-\alpha_{1}} d \alpha_{2} \frac{1}{\left[\alpha_{1} A+\alpha_{2} B+\left(1-\alpha_{1}-\alpha_{2}\right) C+i \epsilon\right]^{3}}
$$

has been used. Hence, the denominator $D$ is given by

$$
\begin{aligned}
& D=\left(1-\alpha_{1}-\alpha_{2}\right)\left[k^{2}+\frac{p^{2}}{4}-m^{2}+\zeta^{\prime} p \cdot k+\zeta 2 k \cdot k_{i}-\gamma^{\prime \prime}\right]+ \\
& +\alpha_{1}\left[\left(\frac{p}{2}+k\right)^{2}-m^{2}\right]+\alpha_{2}\left[\left(\frac{p}{2}-k\right)^{2}-m^{2}\right]= \\
& =k^{2}+\frac{p^{2}}{4}-m^{2}+p \cdot k z^{\prime \prime}+2 k \cdot k_{i} z^{\prime}-\gamma^{\prime}
\end{aligned}
$$

with $z^{\prime \prime}=\zeta^{\prime}\left(1-\alpha_{1}-\alpha_{2}\right)+\alpha_{1}-\alpha_{2}$ and $z^{\prime}=\zeta\left(1-\alpha_{1}-\alpha_{2}\right)$ belonging to $[-1,1]$ and $\gamma^{\prime}=\gamma^{\prime \prime}\left(1-\alpha_{1}-\alpha_{2}\right)$. Then, the vertex function becomes

$$
\left\langle k^{\mu}\left|G_{0}(p) T(p)\right| k_{i}^{\mu}\right\rangle=-i \int_{-1}^{1} d z^{\prime} \int_{-1}^{1} d z^{\prime \prime} \int_{-\infty}^{\infty} d \gamma^{\prime} \frac{g^{(+)}\left(\gamma^{\prime}, z^{\prime}, z^{\prime \prime}\right)}{\left[k^{2}+\frac{p^{2}}{4}-m^{2}+p \cdot k z^{\prime \prime}+2 k \cdot k_{i} z^{\prime}-\gamma^{\prime}+i \epsilon\right]^{3}}
$$


where a factor $(-i)$ has been inserted for convenience (cf [11] for the bound state) and

$$
g^{(+)}\left(\gamma^{\prime}, z^{\prime}, z^{\prime \prime}\right)=i \int_{0}^{1} d \alpha_{1} \int_{0}^{1-\alpha_{1}} d a \frac{1}{a^{3}} \widetilde{\mathcal{G}}^{+}\left(\frac{\gamma^{\prime}}{a}, \frac{z^{\prime}}{a}, \frac{z^{\prime \prime}}{a}\right)
$$

with $a=1-\alpha_{1}-\alpha_{2}$. The vanishing behavior of $\widetilde{\mathcal{G}}^{+}$for values of its arguments at infinity makes finite the twofold integration.

A similar representation can be introduced for the scattering state by using Eq. (62), with the same power as in the case of the bound state analyzed in Refs. [11, 12], viz

$$
\begin{aligned}
& \Phi^{(+)}(k, p)=(2 \pi)^{4} \delta^{(4)}\left(k-k_{i}\right)+ \\
& -i \int_{-1}^{1} d z^{\prime} \int_{-1}^{1} d z^{\prime \prime} \int_{-\infty}^{\infty} d \gamma^{\prime} \frac{g^{(+)}\left(\gamma^{\prime}, z^{\prime}, z^{\prime \prime}\right)}{\left[\gamma^{\prime}+m^{2}-\frac{1}{4} M^{2}-k^{2}-p \cdot k z^{\prime \prime}-2 k \cdot k_{i} z^{\prime}-i \epsilon\right]^{3}}= \\
& =(2 \pi)^{4} \delta^{(4)}\left(k-k_{i}\right)-i \int_{-1}^{1} d z^{\prime} \int_{-1}^{1} d z^{\prime \prime} \int_{-\infty}^{\infty} d \gamma^{\prime} \times \\
& \frac{g^{(+)}\left(\gamma^{\prime}, z^{\prime}, z^{\prime \prime}\right)}{\left[\gamma^{\prime}+\gamma+\kappa^{2}-k^{-}\left(k^{+}+\frac{M}{2} z^{\prime \prime}-\frac{M}{2} z_{i} z^{\prime}\right)-k^{+}\left(\frac{M}{2} z^{\prime \prime}+k_{i}^{-} z^{\prime}\right)+2 z^{\prime} \cos \theta \sqrt{\gamma \gamma_{i}}-i \epsilon\right]^{3}}
\end{aligned}
$$

where we adopt the frame $\tilde{p}=\{M, \mathbf{0}\}$. Following the formalism of Ref. [11, 12], $z_{i}=$ $-2 k_{i}^{+} / M\left(1 \geq\left|z_{i}\right|\right.$ since the incoming particles have positive longitudinal momenta, i.e. $\left.p^{+} / 2 \pm k_{i}^{+} \geq 0\right), \cos \theta=\widehat{\mathbf{k}}_{\perp} \cdot \widehat{\mathbf{k}}_{i \perp}, \gamma=\left|\mathbf{k}_{\perp}\right|^{2}$ and $\gamma_{i}=\left|\mathbf{k}_{i \perp}\right|^{2}$. It should be pointed out that for the variable $\gamma^{\prime}$ a lower bound different from $-\infty$ could be possible, as discussed in Sect. IV] (below Eq. (100)).

The 3D LF scattering wave function is given by $\left(\psi^{(+)}=\sqrt{2} \psi_{n=2 / p}^{(+)}\right)$

$$
\begin{aligned}
& \psi^{(+)}(z, \gamma, \cos \theta)=p^{+} \frac{\left(1-z^{2}\right)}{4} \int \frac{d k^{-}}{2 \pi} \Phi^{(+)}(k, p)= \\
& =p^{+} \frac{\left(1-z^{2}\right)}{4}(2 \pi)^{3} \delta^{(3)}\left(\tilde{k}-\tilde{k}_{i}\right)+\frac{\left(1-z^{2}\right)}{4} \int_{-1}^{1} d z^{\prime} \times \\
& \int_{-\infty}^{\infty} d \gamma^{\prime} \frac{g^{(+)}\left(\gamma^{\prime}, z^{\prime}, z ; \gamma_{i}, z_{i}\right)}{\left[\gamma^{\prime}+\gamma+z^{2} m^{2}+\left(1-z^{2}\right) \kappa^{2}+\frac{M}{2} z z^{\prime}\left(\frac{M}{2} z_{i}+k_{i}^{-}\right)+2 z^{\prime} \cos \theta \sqrt{\gamma \gamma_{i}}-i \epsilon\right]^{2}}
\end{aligned}
$$

where the dependence upon the initial variables $\left\{\gamma_{i}, z_{i}\right\}$ in $g^{(+)}$has been made explicit, for the sake of clarity. The result in Eq. (65) can be obtained by integrating over $k^{-}$the singular integral in Eq. (64) as follows (cf Appendix A in Ref. [11])

$$
\begin{aligned}
& \int_{-\infty}^{\infty} \frac{d k^{-}}{2 \pi} \frac{1}{\left[\gamma^{\prime}+\gamma+\kappa^{2}-k^{-}\left(k^{+}+\frac{M}{2} z^{\prime \prime}-\frac{M}{2} z_{i} z^{\prime}\right)-k^{+}\left(\frac{M}{2} z^{\prime \prime}+k_{i}^{-} z^{\prime}\right)+2 z^{\prime} \cos \theta \sqrt{\gamma \gamma_{i}}-i \epsilon\right]^{3}}= \\
& =\frac{i}{2} \frac{\delta\left(k^{+}+\frac{M}{2} z^{\prime \prime}-\frac{M}{2} z_{i} z^{\prime}\right)}{\left[\gamma^{\prime}+\gamma+\kappa^{2}-k^{+}\left(\frac{M}{2} z^{\prime \prime}+k_{i}^{-} z^{\prime}\right)+2 z^{\prime} \cos \theta \sqrt{\gamma \gamma_{i}}-i \epsilon\right]^{2}}= \\
& =\frac{i}{M} \frac{\delta\left(-z+z^{\prime \prime}-z_{i} z^{\prime}\right)}{\left[\gamma^{\prime}+\gamma+z^{2} m^{2}+\left(1-z^{2}\right) \kappa^{2}+\frac{M}{2} z z^{\prime}\left(\frac{M}{2} z_{i}+k_{i}^{-}\right)+2 z^{\prime} \cos \theta \sqrt{\gamma \gamma_{i}}-i \epsilon\right]^{2}}
\end{aligned}
$$


where $z=-2 k^{+} / M$, with $1 \geq|z|$ (from the request of dealing with particles only, i.e. $p^{+} / 2 \pm k^{+} \geq 0$ in the valence wave-function).

It should be pointed out that the new term, $k \cdot k_{i}$, in the denominator of Eq. (64) does not produce substantial differences in the formal treatment that we applied to the boundstate case (cf Eq. (45)). Therefore, performing analogous formal steps, one obtains an inhomogeneous integral equation for the Nakanishi weight function, $g^{(+)}\left(\gamma, z, z^{\prime} ; \gamma_{i}, z_{i}\right)$. In particular, the inhomogeneous integral equation is obtained by inserting (64) in both sides of the following 4D equation, obtained from Eq. (11)

$$
\begin{aligned}
& {\left[\Phi^{(+)}(k, p)-(2 \pi)^{4} \delta^{(4)}\left(k-k_{i}\right)\right]=G_{0}^{(12)}(k, p) i \mathcal{K}\left(k, k_{i}, p\right)+} \\
& +G_{0}^{(12)}(k, p) \int \frac{d^{4} k^{\prime}}{(2 \pi)^{4}} i \mathcal{K}\left(k, k^{\prime}, p\right)\left[\Phi^{(+)}\left(k^{\prime}, p\right)-(2 \pi)^{4} \delta^{(4)}\left(k^{\prime}-k_{i}\right)\right]
\end{aligned}
$$

and then by projecting onto the LF plane, as shown in Eq. (26) for the bound states, viz

$$
\begin{aligned}
& \int \frac{d k^{-}}{2 \pi}\left[\Phi^{(+)}(k, p)-(2 \pi)^{4} \delta^{(4)}\left(k-k_{i}\right)\right]=\int \frac{d k^{-}}{2 \pi} G_{0}^{(12)}(k, p) i \mathcal{K}\left(k, k_{i}, p\right)+ \\
& +\int \frac{d k^{-}}{2 \pi} G_{0}^{(12)}(k, p) \int \frac{d^{4} k^{\prime}}{(2 \pi)^{4}} i \mathcal{K}\left(k, k^{\prime}, p\right)\left[\Phi^{(+)}\left(k^{\prime}, p\right)-(2 \pi)^{4} \delta^{(4)}\left(k^{\prime}-k_{i}\right)\right]
\end{aligned}
$$

After introducing Eq. (64) in Eq. (68) and integrating over $k^{-}$, one obtains the following integral equation for the Nakanishi amplitude, $g^{(+)}\left(\gamma, z^{\prime}, z ; \gamma_{i}, z_{i}\right)$, without angular momentum decomposition, viz

$$
\begin{aligned}
& \int_{-1}^{1} d z^{\prime} \int_{-\infty}^{\infty} d \gamma^{\prime} \frac{g^{(+)}\left(\gamma^{\prime}, z^{\prime}, z ; \gamma_{i}, z_{i}\right)}{\left[\gamma^{\prime}+\gamma+z^{2} m^{2}+\left(1-z^{2}\right) \kappa^{2}+\frac{M}{2} z z^{\prime}\left(\frac{M}{2} z_{i}+k_{i}^{-}\right)+2 z^{\prime} \cos \theta \sqrt{\gamma \gamma_{i}}-i \epsilon\right]^{2}}= \\
& =\mathcal{I}^{L F}\left(\gamma, z ; \gamma_{i}, z_{i}, \cos \theta\right)+ \\
& +\int_{-\infty}^{\infty} d \gamma^{\prime} \int_{-1}^{1} d \zeta \int_{-1}^{1} d \zeta^{\prime} V_{s}^{L F}\left(\gamma, z ; \gamma_{i}, z_{i}, \gamma^{\prime}, \zeta, \zeta^{\prime}, \cos \theta\right) g^{(+)}\left(\gamma^{\prime}, \zeta, \zeta^{\prime} ; \gamma_{i}, z_{i}\right) .
\end{aligned}
$$

where the inhomogeneous term is given by

$$
\begin{aligned}
& \mathcal{I}^{L F}\left(\gamma, z ; \gamma_{i}, z_{i}, \cos \theta\right)=p^{+} \int \frac{d k^{-}}{2 \pi} G_{0}^{(12)}(k, p) i \mathcal{K}\left(k, k_{i}, p\right)= \\
& =-p^{+} \int \frac{d k^{-}}{2 \pi} \frac{1}{\left[\left(\frac{p}{2}+k\right)^{2}-m^{2}+i \epsilon\right]} \frac{1}{\left[\left(\frac{p}{2}-k\right)^{2}-m^{2}+i \epsilon\right]} i \mathcal{K}\left(k, k_{i}, p\right)
\end{aligned}
$$

and the kernel $V_{s}^{L F}$ is related to the kernel $i \mathcal{K}$ of the BS equation by (cf Eq. (51) for the bound state)

$$
\begin{aligned}
& V_{s}^{L F}\left(\gamma, z ; ; \gamma_{i}, z_{i}, \gamma^{\prime}, \zeta, \zeta^{\prime}, \cos \theta\right)=i p^{+} \int_{-\infty}^{\infty} \frac{d k^{-}}{2 \pi} G_{0}^{(12)}(k, p) \times \\
& \int \frac{d^{4} k^{\prime \prime}}{(2 \pi)^{4}} \frac{i \mathcal{K}\left(k, k^{\prime \prime}, p\right)}{\left[k^{\prime \prime 2}-\kappa^{2}+p \cdot k^{\prime \prime} \zeta^{\prime}+2 k^{\prime \prime} \cdot k_{i} \zeta-\gamma^{\prime}+i \epsilon\right]^{3}}
\end{aligned}
$$


It is worth noting that, modulo the value of $\kappa^{2}$, one formally has for the S-wave

$$
\lim _{\zeta \rightarrow 0} V_{s}^{L F}\left(\gamma, z ; \gamma_{i}, z_{i}, \gamma^{\prime}, \zeta, \zeta^{\prime}, \cos \theta\right)=V_{b}^{L F}\left(\gamma, z ; \gamma^{\prime}, \zeta^{\prime}\right)
$$

Notice that the dependence upon $\gamma_{i}$ and $z_{i}$ is washed out by putting $\zeta=0$. (see subsct. $\mathrm{VC}$ for a discussion in ladder approximation).

\section{The scattering amplitude}

In order to complete the theoretical analysis, let us present the relation between the scattering amplitude and the Nakanishi weight function. For the sake of simplicity, the frame where the $z$-axis is perpendicular to the scattering plane has been chosen, then the scattering amplitude $f(s, \theta)$ (with $s=M^{2}$ ), can be calculated from the BS amplitude. As a matter of fact, one has (cf [39])

$$
\begin{aligned}
& f(s, \theta)=\frac{1}{k_{r}} \sum_{\ell}(2 \ell+1) e^{i \delta_{\ell}} \sin \delta_{\ell} P_{\ell}(\cos \theta)= \\
& =-\frac{1}{M 8 \pi} \lim _{k^{\prime} \rightarrow k_{f}}\left\langle p_{1}^{\prime}, p_{2}^{\prime}|i \mathcal{K}(p)| \Phi^{(+)} ; p, k_{i}\right\rangle= \\
& =-\frac{1}{M 8 \pi} \lim _{k^{\prime} \rightarrow k_{f}}\left\langle k^{\prime}, p\left|G_{0}^{-1}(p)\right| \Phi^{(+)} ; p, k_{i}\right\rangle
\end{aligned}
$$

where $k_{r}=\sqrt{s / 4-m^{2}}, k^{\prime}=\left(p_{1}^{\prime}-p_{2}^{\prime}\right) / 2=, p_{1}^{\prime}+p_{2}^{\prime}=p$ and the orthogonality of the plane waves has been adopted (cf Eq. (10). By using the LF projection method (Refs. [24, 25, 28]), one can move from the 4D Minkowski space to the 3D LF hyperplane, simplifying the analytic integration, without approximations. Then, one can rewrite the scattering amplitude in terms of the LF 3D scattering wave function as follows

$$
\begin{aligned}
& f(s, \theta)=-\frac{1}{M 8 \pi} \lim _{k^{\prime} \rightarrow k_{f}}\left\langle k^{\prime}, p\left|G_{0}^{-1}(p)\right| \Phi^{(+)} ; p, k_{i}\right\rangle= \\
& =-\frac{1}{M 8 \pi} \lim _{\tilde{k}^{\prime} \rightarrow \tilde{k}_{f}}\left\langle\tilde{k}^{\prime}\left|\bar{\Pi}_{0}(p) G_{0}^{-1}(p) \Pi(p)\right| \phi_{L F}^{(+)} ; p, \tilde{k}_{i}\right\rangle
\end{aligned}
$$

where, from Eqs. (40) and (42), one has

$$
\left|p, k^{\prime}\right\rangle=\Pi_{0}\left|\tilde{k}^{\prime}\right\rangle \quad, \quad\left|\Phi^{(+)} ; p, k_{i}\right\rangle=\Pi(p)\left|\phi_{L F}^{(+)} ; p, \tilde{k}_{i}\right\rangle
$$

Recalling that (see Eqs. (33) and (39), and Ref. [28])

$$
\begin{aligned}
& \bar{\Pi}_{0}(p) G_{0}^{-1}(p) \Pi(p)=\Pi_{0}(p) G_{0}^{-1}(p)\left[1+\Delta_{0}(p) W(p)\right] \Pi_{0}(p) \\
& =\bar{\Pi}_{0}(p) G_{0}^{-1}(p) \Pi_{0}(p)=g_{0}^{-1}(p)
\end{aligned}
$$


since

$$
\bar{\Pi}_{0}(p) G_{0}(p)^{-1} \Delta_{0}(p)=\bar{\Pi}_{0}(p)-\bar{\Pi}_{0}(p) G_{0}(p)^{-1} \Pi_{0}(p) g_{0}(p) \bar{\Pi}_{0}(p)=0
$$

one can write

$$
f(s, \theta)=-\frac{1}{M 8 \pi} \lim _{\tilde{k}^{\prime} \rightarrow \tilde{k}_{f}}\left\langle\tilde{k}^{\prime}\left|g_{0}^{-1}(p)\right| \phi_{L F}^{(+)} ; p, \tilde{k}_{i}\right\rangle
$$

Finally, by inserting Eq. (36) one gets

$$
\begin{aligned}
& f(s, \theta)=\frac{i}{M 8 \pi} \lim _{(\gamma, z) \rightarrow\left(\gamma_{f}, z_{f}\right)} \frac{p^{+}}{4}\left(1-z^{2}\right)\left(M^{2}-4 \frac{m^{2}+\gamma}{1-z^{2}}\right) \phi_{L F}^{(+)}(z, \gamma, \cos \theta)= \\
& =\frac{i}{M 8 \pi} \lim _{(\gamma, z) \rightarrow\left(\gamma_{f}, z_{f}\right)}\left(M^{2}-4 \frac{m^{2}+\gamma}{1-z^{2}}\right) \psi^{(+)}(z, \gamma, \cos \theta),
\end{aligned}
$$

where $\psi^{(+)}$is given by Eq. (65) and the dependence upon $\gamma_{i}=\left|\mathbf{k}_{i \perp}\right|^{2}$ is understood.

Equation (79) can be written in terms of the Nakanishi representation by using i) the distorted part of $\psi^{(+)}$and ii) Eq. (69), viz

$$
\begin{aligned}
& f(s, \theta)=-\frac{i}{M 8 \pi} \lim _{(\gamma, z) \rightarrow\left(\gamma_{f}, z_{f}\right)}\left[\gamma+\left(1-z^{2}\right) \kappa^{2}+z^{2} m^{2}\right] \quad\left[\mathcal{I}^{L F}\left(\gamma, z ; \gamma_{i}, z_{i}\right)+\right. \\
& \left.+\int_{-\infty}^{\infty} d \gamma^{\prime} \int_{-1}^{1} d \zeta \int_{-1}^{1} d \zeta^{\prime} V_{s}^{L F}\left(\gamma, z ; \gamma_{i}, z_{i}, \gamma^{\prime}, \zeta, \zeta^{\prime}, \cos \theta\right) g^{(+)}\left(\gamma^{\prime}, \zeta, \zeta^{\prime} ; \gamma_{i}, z_{i}\right)\right]
\end{aligned}
$$

where $\gamma_{f}=\gamma_{i}$ and $z_{f}=z_{i}$. Notice that the factor $\gamma+\left(1-z^{2}\right) \kappa^{2}+z^{2} m^{2}$ vanishing for $(\gamma, z) \rightarrow\left(\gamma_{f}, z_{f}\right)$, is canceled out by the corresponding one in $\mathcal{I}^{L F}$ and $V_{s}^{L F}$. This will be illustrated in the next section within the ladder approximation.

\section{SCATTERING STATES IN LADDER APPROXIMATION}

In this Section, we present the ladder approximation of the integral equation (69), in order to determine the corresponding Nakanishi amplitude, $g_{L}^{(+)}\left(\gamma^{\prime}, z^{\prime}, z ; \gamma_{i}, z_{i}\right)$. We also illustrate how uniqueness can be explicitly exploited in order to get a simpler integral equation, but with a more elaborated kernel. 


\section{A. The Nakanishi weight function integral equation}

In ladder approximation (starting from now, we drop the superscript $L F$ to simplify the notation), Eq. (69) reads

$$
\begin{aligned}
& \int_{-1}^{1} d z^{\prime} \int_{-\infty}^{\infty} d \gamma^{\prime} \frac{g_{L}^{(+)}\left(\gamma^{\prime}, z^{\prime}, z ; \gamma_{i}, z_{i}\right)}{\left[\gamma^{\prime}+\gamma+z^{2} m^{2}+\left(1-z^{2}\right) \kappa^{2}+\frac{M}{2} z z^{\prime}\left(\frac{M}{2} z_{i}+k_{i}^{-}\right)+2 z^{\prime} \cos \theta \sqrt{\gamma \gamma_{i}}-i \epsilon\right]^{2}}= \\
& =\mathcal{I}^{(L)}\left(\gamma, z ; \gamma_{i}, z_{i}, \cos \theta\right)+ \\
& +\int_{-\infty}^{\infty} d \gamma^{\prime \prime} \int_{-1}^{1} d \zeta \int_{-1}^{1} d \zeta^{\prime} V_{s}^{(L)}\left(\gamma, z ; \gamma_{i}, z_{i}, \gamma^{\prime \prime}, \zeta, \zeta^{\prime}, \cos \theta\right) g^{(+)}\left(\gamma^{\prime \prime}, \zeta, \zeta^{\prime} ; \gamma_{i}, z_{i}\right) .
\end{aligned}
$$

where $\mathcal{I}^{(L)}$ is given by (see Appendix $\mathrm{A}$ for details)

$$
\mathcal{I}^{(L)}\left(\gamma, z ; \gamma_{i}, z_{i}, \cos \theta\right)=g^{2} \frac{1}{\left[\gamma+\left(1-z^{2}\right) \kappa^{2}+z^{2} m^{2}-i \epsilon\right]} \mathcal{G}^{(L)}\left(\gamma, z ; \gamma_{i}, z_{i}, \cos \theta\right)
$$

with

$$
\begin{aligned}
& \mathcal{G}^{(L)}\left(\gamma, z ; \gamma_{i}, z_{i}, \cos \theta\right)=\frac{\theta\left(z-z_{i}\right)(1-z)}{\beta\left(z, z_{i}\right)+\gamma\left(1-z_{i}\right)-2(1-z) \cos \theta \sqrt{\gamma \gamma_{i}}-i \epsilon}+ \\
& +\frac{\theta\left(z_{i}-z\right)(1+z)}{\beta\left(-z,-z_{i}\right)+\gamma\left(1+z_{i}\right)-2(1+z) \cos \theta \sqrt{\gamma \gamma_{i}}-i \epsilon}
\end{aligned}
$$

and

$$
\beta\left(z, z_{i}\right)=(1-z)\left[\mu^{2}+\frac{M^{2}}{4}(1-z)\left(1+z_{i}\right)-2 m^{2}\right]+\left(1-z_{i}\right) m^{2}
$$

In Eq. (81), $V_{s}^{(L)}$ (cf Appendix $\mathrm{B}$ for details) is given by

$$
\begin{aligned}
& V_{s}^{(L)}\left(\gamma, z ; \gamma_{i}, z_{i}, \gamma^{\prime \prime}, \zeta, \zeta^{\prime}, \cos \theta\right)=\frac{g^{2}}{2(4 \pi)^{2}} \frac{1}{\left[\gamma+\left(1-z^{2}\right) \kappa^{2}+z^{2} m^{2}-i \epsilon\right]} \times \\
& \int_{0}^{1} d v v^{2} \mathcal{F}\left(v, \gamma, z ; \gamma^{\prime \prime}, \zeta, \zeta^{\prime}, \cos \theta\right)
\end{aligned}
$$

where (dropping the dependence upon the external variables $z_{i}$, and $\gamma_{i}$ for the sake of simplicity)

$$
\mathcal{F}\left(v, \gamma, z ; \gamma^{\prime \prime}, \zeta, \zeta^{\prime}, \cos \theta\right)=\mathcal{C}\left(v, \gamma, z ; \gamma^{\prime \prime}, \zeta, \zeta^{\prime}, z_{i}, \cos \theta\right)+\mathcal{C}\left(v, \gamma,-z ; \gamma^{\prime \prime}, \zeta,-\zeta^{\prime},-z_{i}, \cos \theta\right)
$$

with

$$
\begin{aligned}
& \mathcal{C}\left(v, \gamma, z ; \gamma^{\prime \prime}, \zeta, \zeta^{\prime}, z_{i}, \cos \theta\right)=\frac{(1+z)^{2}}{X^{2}\left(v, z_{i}, \zeta, \zeta^{\prime}\right)} \times \\
& \frac{\theta\left(\zeta^{\prime}-z-z_{i} \zeta\right)}{\left[\gamma+z^{2} m^{2}+\kappa^{2}\left(1-z^{2}\right)+\Gamma\left(v, z, z_{i}, \zeta, \zeta^{\prime}, \gamma^{\prime \prime}\right)+Z\left(z, \zeta, \zeta^{\prime} ; z_{i}\right)\left[\frac{M^{2}}{2} z z_{i}+2 \cos \theta \sqrt{\gamma \gamma_{i}}\right]-i \epsilon\right]^{2}}
\end{aligned}
$$


In Eq. (87), one has

$$
\begin{aligned}
& X\left(v, z_{i}, \zeta, \zeta^{\prime}\right)=v(1-v)\left(1+\zeta^{\prime}-z_{i} \zeta\right) \\
& \Gamma\left(v, z, z_{i}, \zeta, \zeta^{\prime}, \gamma^{\prime \prime}\right)=\frac{(1+z)}{\left(1+\zeta^{\prime}-z_{i} \zeta\right)}\left\{\frac{v}{(1-v)}\left[\zeta^{\prime 2} \frac{M^{2}}{4}+\kappa^{2}\left(1+\zeta^{2}\right)+\gamma^{\prime \prime}\right]+\frac{\mu^{2}}{v}+\gamma^{\prime \prime}\right\} \\
& Z\left(z, \zeta, \zeta^{\prime} ; z_{i}\right)=\zeta \frac{(1+z)}{\left(1+\zeta^{\prime}-z_{i} \zeta\right)}
\end{aligned}
$$

Collecting the above results, one obtains the following integral equations for the Nakanishi amplitude in ladder approximation, $g_{L}^{(+)}$,

$$
\begin{aligned}
& \int_{-1}^{1} d z^{\prime} \int_{-\infty}^{\infty} d \gamma^{\prime} \frac{g_{L}^{(+)}\left(\gamma^{\prime}, z^{\prime}, z ; \gamma_{i}, z_{i}\right)}{\left[\gamma^{\prime}+\gamma+z^{2} m^{2}+\left(1-z^{2}\right) \kappa^{2}+\frac{M^{2}}{2} z z^{\prime} z_{i}+2 z^{\prime} \cos \theta \sqrt{\gamma \gamma_{i}}-i \epsilon\right]^{2}}= \\
& =g^{2} \frac{1}{\left[\gamma+\left(1-z^{2}\right) \kappa^{2}+z^{2} m^{2}-i \epsilon\right]} \mathcal{G}^{(L)}\left(\gamma, z ; \gamma_{i}, z_{i}, \cos \theta\right)+ \\
& +\frac{g^{2}}{2(4 \pi)^{2}} \frac{1}{\left[\gamma+\left(1-z^{2}\right) \kappa^{2}+z^{2} m^{2}-i \epsilon\right]} \int_{-\infty}^{\infty} d \gamma^{\prime \prime} \int_{-1}^{1} d \zeta \int_{-1}^{1} d \zeta^{\prime} \times \\
& \int_{0}^{1} d v v^{2} \mathcal{F}\left(v, \gamma, z ; \gamma^{\prime \prime}, \zeta, \zeta^{\prime}, \cos \theta\right) g_{L}^{(+)}\left(\gamma^{\prime \prime}, \zeta, \zeta^{\prime} ; \gamma_{i}, z_{i}\right)
\end{aligned}
$$

A solution of Eq. (89), obtained by retaining only the inhomogeneous term and assuming the uniqueness of the solution, is discussed in the following Section. Such a solution can yield some insights on the the analytic structure of the ladder approximation of the equation for determining the Nakanishi amplitude $g_{L}^{(+)}$, i.e. Eq. (89). In particular, one could argue that a finite lower bound for the variable $\gamma^{\prime}$ could exist in order to reproduce the analytic structure of the inhomogeneous term. In particular, the inhomogeneous term is proportional to the global propagator

$$
\frac{1}{M^{2}-\frac{4\left(m^{2}+\gamma\right)}{\left(1-z^{2}\right)}+i \epsilon}=-\frac{1}{\gamma+\left(1-z^{2}\right) \kappa^{2}+z^{2} m^{2}-i \epsilon}
$$

that generates a cut starting at $\gamma=-\left(1-z^{2}\right) \kappa^{2}-z^{2} m^{2}$. The lowest value is $\gamma_{\text {min }}=-m^{2} \leq 0$ recalling that $z^{2} \in[0,1]$. Therefore, since $\gamma=\left|\mathbf{k}_{\perp}\right|^{2}$, the lowest bound is zero.

To conclude this subsection, it is shown the scattering amplitude, Eq. (80), in ladder approximation,viz

$$
\begin{aligned}
& f^{(L)}(s, \theta)=-i \frac{g^{2}}{M 8 \pi}\left[\mathcal{G}^{(L)}\left(\gamma_{f}, z_{f} ; \gamma_{i}, z_{i}, \cos \theta\right)+\frac{1}{2^{5} \pi^{2}} \int_{-\infty}^{\infty} d \gamma^{\prime \prime} \int_{-1}^{1} d \zeta \int_{-1}^{1} d \zeta^{\prime} \times\right. \\
& \left.\int_{0}^{1} d v v^{2} \mathcal{F}\left(v, \gamma_{f}, z_{f} ; \gamma^{\prime \prime}, \zeta, \zeta^{\prime}, \cos \theta\right) g_{L}^{(+)}\left(\gamma^{\prime \prime}, \zeta, \zeta^{\prime} ; \gamma_{i}, z_{i}\right)\right]
\end{aligned}
$$


where $\mathcal{G}^{(L)}$ and $\mathcal{F}$ are given in Eqs. (83) and (86), respectively.

\section{B. Applying uniqueness to the integral equation}

Eq. (89) can be rewritten in such a way that the denominator appearing in the lhs can be present in both the terms in the rhs. This allow to explicitly use the uniqueness of the solution of the integral equation and to obtain a simpler equation, as shown in what follows.

By using the standard Feynman trick, one can rewrite $\mathcal{I}^{(L)}$ in a useful form for the following elaboration, namely (see Appendix A for a detailed discussion)

$$
\begin{aligned}
& \mathcal{I}^{(L)}\left(\gamma, z ; \gamma_{i}, z_{i}, \cos \theta\right)=g^{2} \int_{-1}^{1} d z^{\prime} \theta\left(-z^{\prime}\right) \int_{-\infty}^{\infty} d \gamma^{\prime} \times \\
& \frac{\delta\left(\gamma^{\prime}-\gamma_{a}\left(z^{\prime}\right)\right)}{\left[\gamma^{\prime}+\gamma+\left(1-z^{2}\right) \kappa^{2}+z^{2} m^{2}+\frac{M^{2}}{2} z z^{\prime} z_{i}+2 z^{\prime} \cos \theta \sqrt{\gamma \gamma_{i}}-i \epsilon\right]^{2}} \times \\
& \left\{\theta\left(z-z_{i}\right) \theta\left[1-z+z^{\prime}\left(1-z_{i}\right)\right]+\theta\left(z_{i}-z\right) \theta\left[1+z+z^{\prime}\left(1+z_{i}\right)\right]\right\}
\end{aligned}
$$

where

$$
\gamma_{a}\left(z^{\prime}\right)=z^{\prime}\left(2 \kappa^{2}-\mu^{2}\right)
$$

As for $V_{s}^{(L)}$, one can proceed through more subtle mathematical steps. This is thoroughly discussed in Appendix B, here the final expression is given. One has

$$
\begin{aligned}
& V_{s}^{(L)}\left(\gamma, z ; \gamma_{i}, z_{i}, \gamma^{\prime \prime}, \zeta, \zeta^{\prime}, \cos \theta\right)=-\frac{g^{2}}{2(4 \pi)^{2}} \times \\
& \int_{-\infty}^{\infty} d \gamma^{\prime} \int_{-1}^{1} d z^{\prime} \frac{1}{\left[\gamma+z^{2} m^{2}+\kappa^{2}\left(1-z^{2}\right)+\gamma^{\prime}+z^{\prime}\left(\frac{M^{2}}{2} z z_{i}+2 \cos \theta \sqrt{\gamma \gamma_{i}}\right)-i \epsilon\right]^{2}} \times \\
& {\left[\frac{(1+z)}{\left(1+\zeta^{\prime}-z_{i} \zeta\right)} \theta\left(\zeta^{\prime}-z-z_{i} \zeta\right) \mathcal{Q}^{\prime}\left(z, z_{i} ; \gamma^{\prime \prime}, \gamma^{\prime}, z^{\prime}, \zeta, \zeta^{\prime}, \mu^{2}\right)+\right.} \\
& \left.+\frac{(1-z)}{\left(1-\zeta^{\prime}+z_{i} \zeta\right)} \theta\left(z-\zeta^{\prime}+z_{i} \zeta\right) \mathcal{Q}^{\prime}\left(-z,-z_{i} ; \gamma^{\prime \prime}, \gamma^{\prime}, z^{\prime}, \zeta,-\zeta^{\prime}, \mu^{2}\right)\right]
\end{aligned}
$$

where

$$
\begin{aligned}
& \mathcal{Q}^{\prime}\left(z, z_{i} ; \gamma^{\prime \prime}, \gamma^{\prime}, z^{\prime}, \zeta, \zeta^{\prime}, \mu^{2}\right)=\theta\left(\frac{1+z}{1+\zeta^{\prime}-z_{i} \zeta}-z^{\prime} \zeta\right) \frac{\theta\left(z^{\prime}\right) \theta(\zeta)-\theta\left(-z^{\prime}\right) \theta(-\zeta)}{z^{\prime}} \times \\
& \Lambda\left(z, \frac{z^{\prime}}{\zeta}, \zeta, \zeta^{\prime} ; \gamma^{\prime \prime}, \gamma^{\prime} ; z_{i}, \mu^{2}\right)
\end{aligned}
$$


where

$$
\begin{aligned}
& \Lambda\left(z, \frac{z^{\prime}}{\zeta}, \zeta, \zeta^{\prime} ; \gamma^{\prime \prime}, \gamma^{\prime} ; z_{i}, \mu^{2}\right)=\sum_{i= \pm} \frac{\partial}{\partial \lambda} y_{i}(0)\left\{\delta\left(y_{i}(0)\right) \frac{y_{i}^{2}(0)}{\left|y_{i}^{2}(0) \mathcal{A}\left(\zeta, \zeta^{\prime}, \gamma^{\prime \prime}, \kappa^{2}\right)-\mu^{2}\right|}+\right. \\
& \left.-\theta\left(y_{i}(0)\right) \frac{2 \mu^{2} y_{i}(0)}{\left(y_{i}^{2}(0) \mathcal{A}\left(\zeta, \zeta^{\prime}, \gamma^{\prime \prime}, \kappa^{2}\right)-\mu^{2}\right)\left|y_{i}^{2}(0) \mathcal{A}\left(\zeta, \zeta^{\prime}, \gamma^{\prime \prime}, \kappa^{2}\right)-\mu^{2}\right|}\right\}
\end{aligned}
$$

with $y_{ \pm}(0)$ solutions of the following second order equation

$$
y^{2} \mathcal{A}\left(\zeta, \zeta^{\prime}, \gamma^{\prime \prime}, \kappa^{2}\right)+y \mathcal{B}\left(z, z^{\prime}, z_{i}, \zeta, \zeta^{\prime}, \gamma^{\prime \prime}, \gamma^{\prime}, \mu^{2}, \lambda\right)+\mu^{2}=0
$$

The dependence upon $z^{\prime} / \zeta \geq 0$ will become clear from what follows.

In Eq. (96) the coefficients are given by

$$
\begin{aligned}
& \mathcal{A}\left(\zeta, \zeta^{\prime}, \gamma^{\prime \prime}, \kappa^{2}\right)=\zeta^{\prime 2} \frac{M^{2}}{4}+\kappa^{2}\left(1+\zeta^{2}\right)+\gamma^{\prime \prime} \\
& \mathcal{B}\left(z, z^{\prime}, z_{i}, \zeta, \zeta^{\prime}, \gamma^{\prime \prime}, \gamma^{\prime}, \mu^{2}, \lambda\right)=\mu^{2}+\gamma^{\prime}-\gamma^{\prime \prime} \frac{\zeta}{z^{\prime}}+\lambda \frac{\left(1+\zeta^{\prime}-z_{i} \zeta\right)}{(1+z)}
\end{aligned}
$$

In Eq. (94), one explicitly has

$$
\begin{aligned}
& y_{ \pm}(0)=\frac{1}{2 \mathcal{A}\left(\zeta, \zeta^{\prime}, \gamma^{\prime \prime}, \kappa^{2}\right)} \times \\
& {\left[-\mathcal{B}\left(z, z^{\prime}, z_{i}, \zeta, \zeta^{\prime}, \gamma^{\prime \prime}, \gamma^{\prime}, \mu^{2}, 0\right) \pm \sqrt{\mathcal{B}^{2}\left(z, z^{\prime}, z_{i}, \zeta, \zeta^{\prime}, \gamma^{\prime \prime}, \gamma^{\prime}, \mu^{2}, 0\right)-4 \mu^{2} \mathcal{A}\left(\zeta, \zeta^{\prime}, \gamma^{\prime \prime}, \kappa^{2}\right)}\right]} \\
& \frac{\partial}{\partial \lambda} y_{i}(0)=\mp \frac{\left(1+\zeta^{\prime}-z_{i} \zeta\right)}{(1+z)} \frac{y_{ \pm}(0)}{\sqrt{\mathcal{B}^{2}\left(z, z^{\prime}, z_{i}, \zeta, \zeta^{\prime}, \gamma^{\prime \prime}, \gamma^{\prime}, \mu^{2}, 0\right)-4 \mu^{2} \mathcal{A}\left(\zeta, \zeta^{\prime}, \gamma^{\prime \prime}, \kappa^{2}\right)}}
\end{aligned}
$$

Notice that for $\lambda=0$, the dependence upon $z_{i}$ in $\mathcal{B}\left(z, z^{\prime}, z_{i}, \zeta, \zeta^{\prime}, \gamma^{\prime \prime}, \gamma^{\prime}, \mu^{2}, 0\right)$ is dummy.

Eq. (94) can be simplified, noting that the first term, proportional to $y_{i}^{2}(0) \delta\left(y_{i}(0)\right)$, does not contribute if $\mu^{2} \neq 0$, while for $\mu^{2}=0$ (Wick-Cutkosky model, see the next Section) the second term, proportional to $\theta\left(y_{i}(0)\right)$, is vanishing.

By using Eq. (91) and Eqs (93), (89) can be rewritten putting in evidence the analytic 
behavior of all the terms, namely

$$
\begin{aligned}
& \int_{-1}^{1} d z^{\prime} \int_{-\infty}^{\infty} d \gamma^{\prime} \frac{g_{L}^{(+)}\left(\gamma^{\prime}, z^{\prime}, z ; \gamma_{i}, z_{i}\right)}{\left[\gamma^{\prime}+\gamma+z^{2} m^{2}+\left(1-z^{2}\right) \kappa^{2}+\frac{M^{2}}{2} z z^{\prime} z_{i}+2 z^{\prime} \cos \theta \sqrt{\gamma \gamma_{i}}-i \epsilon\right]^{2}}= \\
& =g^{2} \int_{-1}^{1} d z^{\prime} \theta\left(-z^{\prime}\right) \int_{-\infty}^{\infty} d \gamma^{\prime} \frac{\delta\left[\gamma^{\prime}-z^{\prime}\left(2 \kappa^{2}-\mu^{2}\right)\right]}{\left[\gamma^{\prime}+\gamma+\left(1-z^{2}\right) \kappa^{2}+z^{2} m^{2}+\frac{M^{2}}{2} z z^{\prime} z_{i}+2 z^{\prime} \cos \theta \sqrt{\gamma \gamma_{i}}-i \epsilon\right]^{2}} \times \\
& \left\{\theta\left(z-z_{i}\right) \theta\left[1-z+z^{\prime}\left(1-z_{i}\right)\right]+\theta\left(z_{i}-z\right) \theta\left[1+z+z^{\prime}\left(1+z_{i}\right)\right]\right\}+ \\
& -\frac{g^{2}}{2(4 \pi)^{2}} \int_{-1}^{1} d z^{\prime} \int_{-\infty}^{\infty} d \gamma^{\prime} \frac{[}{\left[\gamma+z^{2} m^{2}+\kappa^{2}\left(1-z^{2}\right)+\gamma^{\prime}+z^{\prime}\left(\frac{M^{2}}{2} z z_{i}+2 \cos \theta \sqrt{\gamma \gamma_{i}}\right)-i \epsilon\right]^{2}} \times \\
& \int_{-1}^{1} d \zeta \int_{-1}^{1} d \zeta^{\prime} \int_{-\infty}^{\infty} d \gamma^{\prime \prime}\left[\frac{(1+z)}{\left(1+\zeta^{\prime}-z_{i} \zeta\right)} \theta\left(\zeta^{\prime}-z-z_{i} \zeta\right) \mathcal{Q}^{\prime}\left(z, z_{i} ; \gamma^{\prime \prime}, \gamma^{\prime}, z^{\prime}, \zeta, \zeta^{\prime}, \mu^{2}\right)+\right. \\
& \left.+\frac{(1-z)}{\left(1-\zeta^{\prime}+z_{i} \zeta\right)} \theta\left(z-\zeta^{\prime}+z_{i} \zeta\right) \mathcal{Q}^{\prime}\left(-z,-z_{i} ; \gamma^{\prime \prime}, \gamma^{\prime}, z^{\prime}, \zeta,-\zeta^{\prime}, \mu^{2}\right)\right] g_{L}^{(+)}\left(\gamma^{\prime \prime}, \zeta, \zeta^{\prime} ; \gamma_{i}, z_{i}\right)
\end{aligned}
$$

From the uniqueness of the solution of the integral equation (99), that we expect once the

Nakanishi theorem for the vertex function ( $\mathrm{cf}$ [10]) is extended to the scattering case, one could write (see also [14])

$$
\begin{aligned}
& g_{L}^{(+)}\left(\gamma^{\prime}, z^{\prime}, z ; \gamma_{i}, z_{i}\right)=g^{2} \theta\left(-z^{\prime}\right) \delta\left[\gamma^{\prime}-z^{\prime}\left(2 \kappa^{2}-\mu^{2}\right)\right]\left\{\theta\left(z-z_{i}\right) \theta\left[1-z+z^{\prime}\left(1-z_{i}\right)\right]+\right. \\
& \left.+\theta\left(z_{i}-z\right) \theta\left[1+z+z^{\prime}\left(1+z_{i}\right)\right]\right\}-\frac{g^{2}}{2(4 \pi)^{2}} \int_{-\infty}^{\infty} d \gamma^{\prime \prime} \int_{-1}^{1} d \zeta \int_{-1}^{1} d \zeta^{\prime} g_{L}^{(+)}\left(\gamma^{\prime \prime}, \zeta, \zeta^{\prime} ; \gamma_{i}, z_{i}\right) \times \\
& {\left[\frac{(1+z)}{\left(1+\zeta^{\prime}-z_{i} \zeta\right)} \theta\left(\zeta^{\prime}-z-z_{i} \zeta\right) \mathcal{Q}^{\prime}\left(z, z_{i} ; \gamma^{\prime \prime}, \gamma^{\prime}, z^{\prime}, \zeta, \zeta^{\prime}, \mu^{2}\right)+\right.} \\
& \left.+\frac{(1-z)}{\left(1-\zeta^{\prime}+z_{i} \zeta\right)} \theta\left(z-\zeta^{\prime}+z_{i} \zeta\right) \mathcal{Q}^{\prime}\left(-z,-z_{i} ; \gamma^{\prime \prime}, \gamma^{\prime}, z^{\prime}, \zeta,-\zeta^{\prime}, \mu^{2}\right)\right]
\end{aligned}
$$

The first term in the rhs of Eq. (100) yields the lowest order approximation to $g_{L}^{(+)}$. The following support for $0 \leq \gamma^{\prime} \leq \mu^{2}+2\left|\kappa^{2}\right|$ can be obtained by inspecting the delta function, combined with the constraint on $z^{\prime}$.

\section{APPLICATIONS}

In this Section, some relevant limiting cases, i) the Nakanishi amplitude for scattering states at zero-energy $\left(\kappa^{2} \rightarrow 0, \mu^{2} \neq 0\right)$, ii) the Nakanishi amplitude for the Wick-Cutkosky model in the continuum $\left(\mu^{2} \rightarrow 0\right.$ and $\left.\kappa^{2} \leq 0\right)$, and iii) a revisiting of the ladder kernel for bound states are illustrated in details. It is worth noting that at the end of the WickCutkosky model subsection, it is presented a formal comparison between the kernel for zero energy, obtained within our approach and the kernel that one can find in Ref. [16], for a 
S-wave bound state, putting a vanishing binding energy energy. It is rewarding to find a successful comparison with our formalism. Moreover, as shown in the last subsection, a new form of the integral equation that determines the Nakanishi amplitude for bound states, in ladder approximation, is obtained. This simple integral equation could suggest a different numerical investigation of the issue.

\section{A. The Nakanishi Integral Equation for the zero-energy scattering}

The choice $z_{i}=\gamma_{i}=0$ yields $\kappa^{2}=-\gamma_{i}-z_{i}^{2} M^{2} / 4=0$, namely a zero-energy scattering, given the physical meaning of $\kappa^{2}=m^{2}-M^{2} / 4$. For such values of $z_{i}$ and $\gamma_{i}$, one can simplify Eq. (99), obtaining the Nakanishi integral equation for the weight function at zero energy. Notice that such a weight amplitude determines the scattering length through Eq. (90). After inserting $z_{i}=\gamma_{i}=\kappa^{2}=0$ in Eq. (99), one gets

$$
\begin{aligned}
& \int_{-1}^{1} d z^{\prime} \int_{-\infty}^{\infty} d \gamma^{\prime} \frac{g_{L}^{(+)}\left(\gamma^{\prime}, z^{\prime}, z ; \gamma_{i}=z_{i}=0\right)}{\left[\gamma^{\prime}+\gamma+z^{2} m^{2}-i \epsilon\right]^{2}}= \\
& =g^{2} \int_{-1}^{1} d z^{\prime} \theta\left(-z^{\prime}\right) \int_{-\infty}^{\infty} d \gamma^{\prime} \frac{\delta\left(\gamma^{\prime}+z^{\prime} \mu^{2}\right)}{\left[\gamma^{\prime}+\gamma+z^{2} m^{2}-i \epsilon\right]^{2}} \times \\
& \left\{\theta(z) \theta\left(1-z+z^{\prime}\right)+\theta(-z) \theta\left(1+z+z^{\prime}\right)\right\}+ \\
& -\frac{g^{2}}{2(4 \pi)^{2}} \int_{-1}^{1} d z^{\prime} \int_{-\infty}^{\infty} d \gamma^{\prime} \frac{1}{\left[\gamma^{\prime}+\gamma+z^{2} m^{2}+-i \epsilon\right]^{2}} \times \\
& \int_{-1}^{1} d \zeta \int_{-1}^{1} d \zeta^{\prime} \int_{-\infty}^{\infty} d \gamma^{\prime \prime}\left[\frac{(1+z)}{\left(1+\zeta^{\prime}\right)} \theta\left(\zeta^{\prime}-z\right) \mathcal{Q}^{\prime}\left(z, z_{i}=0 ; \gamma^{\prime \prime}, \gamma^{\prime}, z^{\prime}, \zeta, \zeta^{\prime}, \mu^{2}\right)+\right. \\
& \left.+\frac{(1-z)}{\left(1-\zeta^{\prime}\right)} \theta\left(z-\zeta^{\prime}\right) \mathcal{Q}^{\prime}\left(-z, z_{i}=0 ; \gamma^{\prime \prime}, \gamma^{\prime}, z^{\prime}, \zeta,-\zeta^{\prime}, \mu^{2}\right)\right] g_{L}^{(+)}\left(\gamma^{\prime \prime}, \zeta, \zeta^{\prime} ; \gamma_{i}=z_{i}=0 \gamma 101\right)
\end{aligned}
$$

One immediately realizes that the integration over $z^{\prime}$ can be performed in the lhs, introducing the suitable S-wave Nakanishi amplitude for the present case. Then, one gets

$$
\begin{aligned}
& \int_{-\infty}^{\infty} d \gamma^{\prime} \frac{g_{0 L}^{(+)}\left(\gamma^{\prime}, z\right)}{\left[\gamma^{\prime}+\gamma+z^{2} m^{2}-i \epsilon\right]^{2}}= \\
& =\frac{g^{2}}{\mu^{2}} \int_{-\infty}^{\infty} d \gamma^{\prime} \frac{\theta\left(\gamma^{\prime}\right)}{\left[\gamma^{\prime}+\gamma+z^{2} m^{2}-i \epsilon\right]^{2}}\left\{\theta(z) \theta\left(1-z-\gamma^{\prime} / \mu^{2}\right)+\theta(-z) \theta\left(1+z-\gamma^{\prime} / \mu^{2}\right)\right\}+ \\
& -\frac{g^{2}}{2(4 \pi)^{2}} \int_{-\infty}^{\infty} d \gamma^{\prime} \frac{1}{\left[\gamma^{\prime}+\gamma+z^{2} m^{2}+-i \epsilon\right]^{2}} \times \\
& \int_{-1}^{1} d \zeta^{\prime} \int_{-\infty}^{\infty} d \gamma^{\prime \prime}\left[\frac{(1+z)}{\left(1+\zeta^{\prime}\right)} \theta\left(\zeta^{\prime}-z\right) \mathcal{T}\left(z, \gamma^{\prime \prime}, \gamma^{\prime}, \zeta^{\prime}, \mu^{2}\right)+\right. \\
& \left.+\frac{(1-z)}{\left(1-\zeta^{\prime}\right)} \theta\left(z-\zeta^{\prime}\right) \mathcal{T}\left(-z, \gamma^{\prime \prime}, \gamma^{\prime},-\zeta^{\prime}, \mu^{2}\right)\right] g_{0 L}^{(+)}\left(\gamma^{\prime \prime}, \zeta^{\prime}\right)
\end{aligned}
$$


where

$$
\begin{aligned}
& g_{0 L}^{(+)}\left(\gamma^{\prime}, z\right)=\int_{-1}^{1} d z^{\prime} g_{L}^{(+)}\left(\gamma^{\prime}, z^{\prime}, z ; \gamma_{i}=z_{i}=0\right) \\
& \int_{-1}^{0} d z^{\prime} \delta\left(\gamma^{\prime}+z^{\prime} \mu^{2}\right)=\frac{1}{\mu^{2}} \theta\left(\gamma^{\prime}\right) \\
& g_{0 L}^{(+)}\left(\gamma^{\prime \prime}, \zeta^{\prime}\right)=\int_{-1}^{1} d \zeta g_{L}^{(+)}\left(\gamma^{\prime \prime}, \zeta, \zeta^{\prime} ; \gamma_{i}=z_{i}=0\right)
\end{aligned}
$$

and the possibility to integrate over $\zeta$ the function $g_{L}^{(+)}\left(\gamma^{\prime \prime}, \zeta, \zeta^{\prime} ; \gamma_{i}=z_{i}=0\right)$ is related to the independence upon $\zeta$ of the function

$$
\mathcal{T}\left(z, \gamma^{\prime \prime}, \gamma^{\prime}, \zeta^{\prime}, \mu^{2}\right)=\int_{-1}^{1} d z^{\prime} \mathcal{Q}^{\prime}\left(z, z_{i}=0 ; \gamma^{\prime \prime}, \gamma^{\prime}, z^{\prime}, \zeta, \zeta^{\prime}, \mu^{2}\right)
$$

As a matter of fact, from Eq. (94) one has

$$
\begin{aligned}
& \mathcal{T}\left(z, \gamma^{\prime \prime}, \gamma^{\prime}, \zeta^{\prime}, \mu^{2}\right)=\int_{-1}^{1} \frac{d z^{\prime}}{z^{\prime}} \theta\left(\frac{1+z}{1+\zeta^{\prime}}-\frac{z^{\prime}}{\zeta}\right)\left[\theta\left(z^{\prime}\right) \theta(\zeta)-\theta\left(-z^{\prime}\right) \theta(-\zeta)\right] \times \\
& \Lambda_{0}\left(z, \frac{z^{\prime}}{\zeta}, \zeta^{\prime}, \gamma^{\prime}, \gamma^{\prime \prime}, \mu^{2}\right)= \\
& =\left\{\theta(\zeta) \int_{0}^{\zeta \frac{(1+z)}{\left(1+\zeta^{\prime}\right)}} \frac{d z^{\prime}}{z^{\prime}}-\theta(-\zeta) \int_{\zeta \frac{(1+z)}{\left(1+\zeta^{\prime}\right)}}^{0} \frac{d z^{\prime}}{z^{\prime}}\right\} \Lambda_{0}\left(z, \frac{z^{\prime}}{\zeta}, \zeta^{\prime}, \gamma^{\prime}, \gamma^{\prime \prime}, \mu^{2}\right)
\end{aligned}
$$

where

$$
\begin{aligned}
& \Lambda_{0}\left(z, \frac{z^{\prime}}{\zeta}, \zeta^{\prime}, \gamma^{\prime}, \gamma^{\prime \prime}, \mu^{2}\right)=\sum_{i= \pm} \frac{\partial}{\partial \lambda} y_{i}(0) \frac{1}{\left|y_{i}^{2}(0) \mathcal{A}_{0}\left(\zeta^{\prime}, \gamma^{\prime \prime}\right)-\mu^{2}\right|} \times \\
& {\left[\delta\left(y_{i}(0)\right) y_{i}^{2}(0)-\theta\left(y_{i}(0)\right) \frac{2 \mu^{2} y_{i}(0)}{y_{i}^{2}(0) \mathcal{A}_{0}\left(\zeta^{\prime}, \gamma^{\prime \prime}\right)-\mu^{2}}\right]}
\end{aligned}
$$

with the dependence upon $z^{\prime} / \zeta$ (always positive !) explained in what follows. From Eqs. (97) and (98), one can explicitly write

$$
\begin{aligned}
& \mathcal{A}_{0}\left(\zeta^{\prime}, \gamma^{\prime \prime}\right)=\zeta^{\prime 2} \frac{M^{2}}{4}+\gamma^{\prime \prime}=\zeta^{\prime 2} m^{2}+\gamma^{\prime \prime} \\
& \mathcal{B}\left(z, z^{\prime}, 0, \zeta, \zeta^{\prime}, \gamma^{\prime}, \gamma^{\prime \prime}, \mu^{2}, 0\right)=\mu^{2}+\gamma^{\prime \prime}-\gamma^{\prime} \frac{\zeta}{z^{\prime}} \\
& y_{ \pm}(0)=\frac{1}{2 \mathcal{A}_{0}\left(\zeta^{\prime}, \gamma^{\prime \prime}\right)} \times \\
& {\left[-\left(\mu^{2}+\gamma^{\prime \prime}-\gamma^{\prime} \frac{\zeta}{z^{\prime}}\right) \pm \sqrt{\left(\mu^{2}+\gamma^{\prime \prime}-\gamma^{\prime} \frac{\zeta}{z^{\prime}}\right)^{2}-4 \mu^{2} \mathcal{A}_{0}\left(\zeta^{\prime}, \gamma^{\prime \prime}\right)}\right]} \\
& \left.\frac{\partial}{\partial \lambda} y_{i}(0)=\mp \frac{\left(1+\zeta^{\prime}\right)}{(1+z)} \frac{\sqrt{\left(\mu^{2}+\gamma^{\prime \prime}-\gamma^{\prime} \frac{\zeta}{z^{\prime}}\right)^{2}-4 \mu^{2} \mathcal{A}_{0}\left(\zeta^{\prime}, \gamma^{\prime \prime}\right)}}{\sqrt{\left(1+x^{2}\right.}}\right]
\end{aligned}
$$


This allows one to single out the dependence upon $\frac{z^{\prime}}{\zeta}$ of the integrand in Eq. (104) and, after introducing the variable

$$
x=\frac{z^{\prime}}{\zeta} \frac{\left(1+\zeta^{\prime}\right)}{(1+z)}
$$

one has

$$
\mathcal{T}\left(z, \gamma^{\prime \prime}, \gamma^{\prime}, \zeta^{\prime}, \mu^{2}\right)=[\theta(\zeta)+\theta(-\zeta)] \int_{0}^{1} \frac{d x}{x} \Lambda_{0}\left(z, x \frac{(1+z)}{\left(1+\zeta^{\prime}\right)}, \zeta^{\prime}, \gamma^{\prime}, \gamma^{\prime \prime}, \mu^{2}\right)
$$

This completes the proof that $\mathcal{T}$ does not depend upon $\zeta$. Finally, from uniqueness, one can rewrite the inhomogeneous integral equation (102) in a simpler form, viz

$$
\begin{aligned}
& g_{0 L}^{(+)}\left(\gamma^{\prime}, z\right)=\frac{g^{2}}{\mu^{2}} \theta\left(\gamma^{\prime}\right)\left[\theta(z) \theta\left(1-z-\gamma^{\prime} / \mu^{2}\right)+\theta(-z) \theta\left(1+z-\gamma^{\prime} / \mu^{2}\right)\right]+ \\
& -\frac{g^{2}}{2(4 \pi)^{2}} \int_{-1}^{1} d \zeta^{\prime} \int_{-\infty}^{\infty} d \gamma^{\prime \prime}\left[\frac{(1+z)}{\left(1+\zeta^{\prime}\right)} \theta\left(\zeta^{\prime}-z\right) \mathcal{T}\left(z, \gamma^{\prime \prime}, \gamma^{\prime}, \zeta^{\prime}, \mu^{2}\right)+\right. \\
& \left.+\frac{(1-z)}{\left(1-\zeta^{\prime}\right)} \theta\left(z-\zeta^{\prime}\right) \mathcal{T}\left(-z, \gamma^{\prime \prime}, \gamma^{\prime},-\zeta^{\prime}, \mu^{2}\right)\right] g_{0 L}^{(+)}\left(\gamma^{\prime \prime}, \zeta^{\prime}\right)
\end{aligned}
$$

Such an equation could have a direct application in the study of the relativistic effects of the scattering length.

\section{B. The Nakanishi amplitude for the Wick-Cutkosky model in the continuum}

The Wick-Cutkosky model [3], 30], namely two massive scalars interacting through a massless one $\left(\mu^{2}=0\right)$ in ladder approximation, can be extended to the scattering case. The integral equation for the Nakanishi amplitude, for this widely adopted model, was known so far only for the bound state case (cf [2, 3, 30] and [16] for a LF approach). Equation (100) becomes

$$
\begin{aligned}
& g_{L W}^{(+)}\left(\gamma^{\prime}, z^{\prime}, z ; \gamma_{i}, z_{i}\right)=g^{2} \theta\left(-z^{\prime}\right) \delta\left(\gamma^{\prime}-2 z^{\prime} \kappa^{2}\right)\left[\theta\left(z-z_{i}\right) \theta\left[1-z+z^{\prime}\left(1-z_{i}\right)\right]+\right. \\
& \left.+\theta\left(z_{i}-z\right) \theta\left[1+z+z^{\prime}\left(1+z_{i}\right)\right]\right]+ \\
& -\frac{g^{2}}{2(4 \pi)^{2}} \int_{-\infty}^{\infty} d \gamma^{\prime \prime} \int_{-1}^{1} d \zeta \int_{-1}^{1} d \zeta^{\prime} g_{L W}^{(+)}\left(\gamma^{\prime \prime}, \zeta, \zeta^{\prime} ; \gamma_{i}, z_{i}\right) \times \\
& {\left[\frac{(1+z)}{\left(1+\zeta^{\prime}-z_{i} \zeta\right)} \theta\left(\zeta^{\prime}-z-z_{i} \zeta\right) \mathcal{Q}^{\prime}\left(z, z_{i} ; \gamma^{\prime \prime}, \gamma^{\prime}, z^{\prime}, \zeta, \zeta^{\prime}, \mu^{2}=0\right)+\right.} \\
& \left.+\frac{(1-z)}{\left(1-\zeta^{\prime}+z_{i} \zeta\right)} \theta\left(z-\zeta^{\prime}+z_{i} \zeta\right) \mathcal{Q}^{\prime}\left(-z,-z_{i} ; \gamma^{\prime \prime}, \gamma^{\prime}, z^{\prime}, \zeta,-\zeta^{\prime}, \mu^{2}=0\right)\right]
\end{aligned}
$$


where

$$
\begin{aligned}
& \mathcal{Q}^{\prime}\left(z, z_{i} ; \gamma^{\prime}, \gamma^{\prime \prime}, z^{\prime}, \zeta, \zeta^{\prime}, \mu^{2}=0\right)=\theta\left(\frac{1+z}{1+\zeta^{\prime}-z_{i} \zeta}-z^{\prime} \zeta\right) \frac{\theta\left(z^{\prime}\right) \theta(\zeta)-\theta\left(-z^{\prime}\right) \theta(-\zeta)}{z^{\prime}} \times \\
& \frac{\partial}{\partial \lambda} y_{0}(0) \delta\left(y_{0}(0)\right) \frac{1}{\left|\mathcal{A}\left(\zeta, \zeta^{\prime}, \gamma^{\prime \prime}\right)\right|}
\end{aligned}
$$

with $y_{0}(0)$ being the non trivial the solution of the following second order equation

$$
\mathcal{A}\left(\zeta, \zeta^{\prime}, \gamma^{\prime \prime}\right) y^{2}+y \mathcal{B}\left(z, z^{\prime}, z_{i}, \zeta, \zeta^{\prime}, \gamma^{\prime \prime}, \mu^{2}=0, \lambda\right)=0
$$

Notice that the trivial solution, $y=0$ for any $\lambda$, does not contribute, due to the presence of $\partial y_{0}(0) / \partial \lambda$. In Eq. (111) the coefficients are given by

$$
\begin{aligned}
& \mathcal{A}\left(\zeta, \zeta^{\prime}, \gamma^{\prime \prime}\right)=\zeta^{\prime 2} \frac{M^{2}}{4}+\kappa^{2}\left(1+\zeta^{2}\right)+\gamma^{\prime \prime} \\
& \mathcal{B}\left(z, z^{\prime}, z_{i}, \zeta, \zeta^{\prime}, \gamma^{\prime \prime}, \mu^{2}=0, \lambda\right)=\gamma^{\prime \prime}-\gamma^{\prime} \frac{\zeta}{z^{\prime}}+\lambda \frac{\left(1+\zeta^{\prime}-z_{i} \zeta\right)}{(1+z)}
\end{aligned}
$$

Then, one explicitly has

$$
\begin{aligned}
& y_{0}(0)=\frac{\gamma^{\prime} \frac{\zeta}{z^{\prime}}-\gamma^{\prime \prime}}{\mathcal{A}\left(\zeta, \zeta^{\prime}, \gamma^{\prime \prime}, \kappa^{2}\right)} \\
& \frac{\partial}{\partial \lambda} y_{0}(0)=-\frac{\left(1+\zeta^{\prime}-z_{i} \zeta\right)}{(1+z)} \frac{1}{\mathcal{A}\left(\zeta, \zeta^{\prime}, \gamma^{\prime \prime}, \kappa^{2}\right)}
\end{aligned}
$$

It has to be pointed out that, if $\mathcal{A}\left(\zeta, \zeta^{\prime}, \gamma^{\prime \prime}\right)=0$, one has only the trivial solution $y=0$ for any $\lambda$.

Equation (109) can be rewritten as follows

$$
\begin{aligned}
& g_{L W}^{(+)}\left(\gamma^{\prime}, z^{\prime}, z ; \gamma_{i}, z_{i}\right)=g^{2} \theta\left(-z^{\prime}\right) \delta\left(\gamma^{\prime}-2 z^{\prime} \kappa^{2}\right)\left[\theta\left(z-z_{i}\right) \theta\left[1-z+z^{\prime}\left(1-z_{i}\right)\right]+\right. \\
& \left.+\theta\left(z_{i}-z\right) \theta\left[1+z+z^{\prime}\left(1+z_{i}\right)\right]\right]+ \\
& +\frac{g^{2}}{2(4 \pi)^{2}} \theta\left(z^{\prime}\right) \int_{-\infty}^{\infty} d \gamma^{\prime \prime} \int_{0}^{1} \frac{d \zeta}{z^{\prime}} \int_{-1}^{1} d \zeta^{\prime} \frac{\delta\left(\gamma^{\prime} \frac{\zeta}{z^{\prime}}-\gamma^{\prime \prime}\right)}{\mathcal{A}\left(\zeta, \zeta^{\prime}, \gamma^{\prime \prime}, \kappa^{2}\right)} g_{L W}^{(+)}\left(\gamma^{\prime \prime}, \zeta, \zeta^{\prime} ; \gamma_{i}, z_{i}\right) \times \\
& \left\{\theta\left(\zeta^{\prime}-z-z_{i} \zeta\right) \theta\left(\frac{1+z}{1+\zeta^{\prime}-z_{i} \zeta}-\frac{z^{\prime}}{\zeta}\right)+\theta\left(z-\zeta^{\prime}+z_{i} \zeta\right) \theta\left(\frac{1-z}{1-\zeta^{\prime}+z_{i} \zeta}-\frac{z^{\prime}}{\zeta}\right)\right\} \\
& -\frac{g^{2}}{2(4 \pi)^{2}} \theta\left(-z^{\prime}\right) \int_{-\infty}^{\infty} d \gamma^{\prime \prime} \int_{-1}^{0} \frac{d \zeta}{z^{\prime}} \int_{-1}^{1} d \zeta^{\prime} \frac{\delta\left(\gamma^{\prime} \frac{\zeta}{z^{\prime}}-\gamma^{\prime \prime}\right)}{\mathcal{A}\left(\zeta, \zeta^{\prime}, \gamma^{\prime \prime}, \kappa^{2}\right)} g_{L W}^{(+)}\left(\gamma^{\prime \prime}, \zeta, \zeta^{\prime} ; \gamma_{i}, z_{i}\right) \times \\
& \left\{\theta\left(\zeta^{\prime}-z-z_{i} \zeta\right) \theta\left(\frac{1+z}{1+\zeta^{\prime}-z_{i} \zeta}-\frac{z^{\prime}}{\zeta}\right)+\theta\left(z-\zeta^{\prime}+z_{i} \zeta\right) \theta\left(\frac{1-z}{1-\zeta^{\prime}+z_{i} \zeta}-\frac{z^{\prime}}{\zeta}\right)\right\}
\end{aligned}
$$

Notice that the extrema of the integration on $\zeta$ in the second and third terms are different. It is easily seen that, in the rhs, the positive $z^{\prime}$ decouples from the negative one, obtaining 
a homogeneous integral equation for $\theta\left(z^{\prime}\right) g_{L W}^{(+)}\left(\gamma^{\prime}, z^{\prime}, z ; \gamma_{i}, z_{i}\right)$ and an inhomogeneous one for $\theta\left(-z^{\prime}\right) g_{L W}^{(+)}\left(\gamma^{\prime}, z^{\prime}, z ; \gamma_{i}, z_{i}\right)$. Therefore, the Nakanishi amplitude for scattering states within the Wick-Cutkosky model is given by the following inhomogeneous integral equation (it is inhomogeneous for matching the boundary condition for scattering states)

$$
\begin{aligned}
& g_{L W}^{(+)}\left(\gamma^{\prime}, z^{\prime}, z ; \gamma_{i}, z_{i}\right)=g^{2} \theta\left(-z^{\prime}\right) \delta\left(\gamma^{\prime}-2 z^{\prime} \kappa^{2}\right)\left[\theta\left(z-z_{i}\right) \theta\left[1-z+z^{\prime}\left(1-z_{i}\right)\right]+\right. \\
& \left.+\theta\left(z_{i}-z\right) \theta\left[1+z+z^{\prime}\left(1+z_{i}\right)\right]\right]+ \\
& -\frac{g^{2}}{2(4 \pi)^{2}} \theta\left(-z^{\prime}\right) \int_{-\infty}^{\infty} d \gamma^{\prime \prime} \int_{-1}^{0} \frac{d \zeta}{z^{\prime}} \int_{-1}^{1} d \zeta^{\prime} \frac{\delta\left(\gamma^{\prime} \frac{\zeta}{z^{\prime}}-\gamma^{\prime \prime}\right)}{\mathcal{A}\left(\zeta, \zeta^{\prime}, \gamma^{\prime \prime}, \kappa^{2}\right)} g_{L W}^{(+)}\left(\gamma^{\prime \prime}, \zeta, \zeta^{\prime} ; \gamma_{i}, z_{i}\right) \times \\
& \left\{\theta\left(\zeta^{\prime}-z-z_{i} \zeta\right) \theta\left(\frac{1+z}{1+\zeta^{\prime}-z_{i} \zeta}-\frac{z^{\prime}}{\zeta}\right)+\theta\left(z-\zeta^{\prime}+z_{i} \zeta\right) \theta\left(\frac{1-z}{1-\zeta^{\prime}+z_{i} \zeta}-\frac{z^{\prime}}{\zeta}\right)\right\}
\end{aligned}
$$

Let us assume the following separable form for

$$
g_{L W}^{(+)}\left(\gamma^{\prime}, z^{\prime}, z ; \gamma_{i}, z_{i}\right)=\theta\left(-z^{\prime}\right) \delta\left(\gamma^{\prime}-2 z^{\prime} \kappa^{2}\right) h_{L W}^{(+)}\left(z^{\prime}, z ; \gamma_{i}, z_{i}\right)
$$

Then, by integrating both sides of Eq. (115) on $\gamma^{\prime}$, one has (recalling that $\zeta / z^{\prime} \geq 0$ )

$$
\begin{aligned}
& h_{L W}^{(+)}\left(z^{\prime}, z ; \gamma_{i}, z_{i}\right)=g^{2}\left\{\theta\left(z-z_{i}\right) \theta\left[1-z+z^{\prime}\left(1-z_{i}\right)\right]+\theta\left(z_{i}-z\right) \theta\left[1+z+z^{\prime}\left(1+z_{i}\right)\right]\right\}+ \\
& -\frac{g^{2}}{2(4 \pi)^{2}} \int_{-1}^{0} \frac{d \zeta}{\zeta} \int_{-1}^{1} d \zeta^{\prime} \frac{1}{\zeta^{\prime 2} \frac{M^{2}}{4}+\kappa^{2}(1+\zeta)^{2}} h_{L W}^{(+)}\left(\zeta, \zeta^{\prime} ; \gamma_{i}, z_{i}\right) \times \\
& \left\{\theta\left(\zeta^{\prime}-z-z_{i} \zeta\right) \theta\left(\frac{1+z}{1+\zeta^{\prime}-z_{i} \zeta}-\frac{z^{\prime}}{\zeta}\right)+\theta\left(z-\zeta^{\prime}+z_{i} \zeta\right) \theta\left(\frac{1-z}{1-\zeta^{\prime}+z_{i} \zeta}-\frac{z^{\prime}}{\zeta}\right)\right\} \quad(116)
\end{aligned}
$$

It is worth noting that the solution $h_{L W}^{(+)}$is perfectly compatible with the separable form we assumed, and that $\zeta^{\prime 2} \frac{M^{2}}{4}+\kappa^{2}(1+\zeta)^{2}=\mathcal{A}\left(\zeta, \zeta^{\prime}, 2 \zeta \kappa^{2}, \kappa^{2}\right)$ is different from zero, as discussed below Eq. (113).

Summarizing, the solution of Eq. (116), together with the separable form mentioned above, allows one to obtain the Nakanishi amplitude for scattering states within the WickCutkosky model in the continuum.

As a formal check of our previous elaboration, in what follows we devise the same kernel, one has for the Wick-Cutkosky model for bound states in the limit $\kappa^{2} \rightarrow 0$. This allows us to make a successful comparison with the result for the S-wave, in the limit of zero-binding energy, that one can get from Ref. [16].

In the limit

$$
\kappa^{2}=m^{2}-\frac{M^{2}}{4}=-\gamma_{i}-z_{i}^{2} \frac{M^{2}}{4} \rightarrow 0
$$


Eq. (116) becomes (dropping the dependence upon $z_{i}=\gamma_{i}=0$ )

$$
\begin{aligned}
& h_{L W}^{(+)}\left(z^{\prime}, z\right)=g^{2}\left[\theta(z) \theta\left[1-z+z^{\prime}\right]+\theta(-z) \theta\left[1+z+z^{\prime}\right]\right]+ \\
& -\frac{g^{2}}{2(4 \pi)^{2}} \int_{-1}^{0} \frac{d \zeta}{\zeta} \int_{-1}^{1} d \zeta^{\prime} \frac{1}{\zeta^{\prime 2} m^{2}} h_{L W}^{(+)}\left(\zeta, \zeta^{\prime}\right) \times \\
& \left\{\theta\left(\zeta^{\prime}-z\right) \theta\left[z^{\prime}-\zeta \frac{(1+z)}{\left(1+\zeta^{\prime}\right)}\right]+\theta\left(z-\zeta^{\prime}\right) \theta\left[z^{\prime}-\zeta \frac{(1-z)}{\left(1-\zeta^{\prime}\right)}\right]\right\}
\end{aligned}
$$

By integrating over $z^{\prime}$ (let us recall that $0 \geq z^{\prime} \geq-1$ ), in order to match the S-wave dependence, one can write

$$
\begin{aligned}
& a_{L W}^{(+)}(z)=g^{2}[\theta(z)(1-z)+\theta(-z)(1+z)]-\frac{g^{2}}{2(4 \pi)^{2}} \int_{-1}^{0} \frac{d \zeta}{\zeta} \int_{-1}^{1} d \zeta^{\prime} \frac{1}{\zeta^{\prime 2} m^{2}} h_{L W}^{(+)}\left(\zeta, \zeta^{\prime}\right) \times \\
& \int_{-1}^{0} d z^{\prime}\left\{\theta\left(\zeta^{\prime}-z\right) \theta\left[z^{\prime}-\zeta \frac{(1+z)}{\left(1+\zeta^{\prime}\right)}\right]+\theta\left(z-\zeta^{\prime}\right) \theta\left[z^{\prime}-\zeta \frac{(1-z)}{\left(1-\zeta^{\prime}\right)}\right]\right\}= \\
& =g^{2}[\theta(z)(1-z)+\theta(-z)(1+z)]+\frac{g^{2}}{2(4 \pi)^{2}} \times \\
& \int_{-1}^{1} d \zeta^{\prime} \frac{1}{\zeta^{\prime 2} m^{2}}\left\{\theta\left(\zeta^{\prime}-z\right) \frac{(1+z)}{\left(1+\zeta^{\prime}\right)}+\theta\left(z-\zeta^{\prime}\right) \frac{(1-z)}{\left(1-\zeta^{\prime}\right)}\right\} a_{L W}^{(+)}\left(\zeta^{\prime}\right)
\end{aligned}
$$

where $0 \leq(1 \pm z) /\left(1 \pm \zeta^{\prime}\right)<1$ and

$$
\begin{aligned}
& a_{L W}^{(+)}(z)=\int_{-1}^{0} d z^{\prime} h_{L W}^{(+)}\left(z^{\prime}, z\right) \\
& a_{L W}^{(+)}\left(\zeta^{\prime}\right)=\int_{-1}^{0} d \zeta h_{L W}^{(+)}\left(\zeta, \zeta^{\prime}\right)
\end{aligned}
$$

The S-wave Nakanishi amplitude is even for $z \rightarrow-z$ transformation.

It should be pointed out that the inhomogeneous term has the same form, when the zero-energy limit is performed first, and the limit $\mu^{2} \rightarrow 0$, as briefly shown below. As a matter of fact, from the inhomogeneous term in Eq. (108) (corresponding to $\kappa^{2} \rightarrow 0$ ), one gets

$$
\begin{aligned}
& \lim _{\mu^{2} \rightarrow 0} \frac{g^{2}}{\mu^{2}} \theta\left(\gamma^{\prime}\right)\left[\theta(z) \theta\left(1-z-\gamma^{\prime} / \mu^{2}\right)+\theta(-z) \theta\left(1+z-\gamma^{\prime} / \mu^{2}\right)\right]= \\
& =g^{2} \delta\left(\gamma^{\prime}\right)[\theta(z)(1-z)+\theta(-z)(1+z)]
\end{aligned}
$$

where the the following representation of the delta function has been used

$$
\lim _{\mu^{2} \rightarrow 0} \frac{1}{\mu^{2}} \theta\left(\gamma^{\prime}\right) \theta\left[\mu^{2}(1-|z|)-\gamma^{\prime}\right]=(1-|z|) \delta\left(\gamma^{\prime}\right)
$$


Such a relation can be deduced by i) introducing the infinitesimal quantity $(1-|z|) \mu^{2}$, ii) folding the previous limit with a generic function $f\left(\gamma^{\prime}\right)$ and iii) integrating over $\gamma^{\prime}$.

As to the kernel, as above anticipated, it coincides with the one given in Ref. [16], for a bound S-wave state, but in the limit of zero binding-energy. This comparison, even if it does not apply to a physically meaningful case, due to the presence of an essential singularity, $\zeta^{\prime 2} \mathrm{~m}^{2}$, gives us confidence in our formalism, since it is able to reproduce the result corresponding to a well known case, after performing the non trivial limits $\mu^{2} \rightarrow 0$ and then $\kappa^{2} \rightarrow 0$.

Finally, it is important to remark that even the non relativistic Coulomb problem, for positive energies, studied through the Lippman-Schwinger equation, has an infrared divergence that must be treated carefully. In the Wick-Cutkosky model this problem is present and has to be addressed to provide meaningful solutions for the relativistic case in the scattering region.

\section{The S-wave bound state in ladder approximation revisited}

The integral equation that determines the Nakanishi amplitude for a S-wave bound state, in ladder approximation, can be rewritten in a simplified form, with respect to the one presented in Ref. [11], once the uniqueness of the solutions is exploited. In particular, one can start with the expression of $V_{s}^{(L)}$ given in Eq. (93), carefully performing the limit $\zeta \rightarrow 0$ and taking a negative value for $\kappa^{2}$ in the final result.

From Ref. [11], one has for a S-wave bound state

$$
\begin{aligned}
& \int_{0}^{\infty} d \gamma^{\prime} \frac{g_{b}\left(\gamma^{\prime}, z ; \kappa^{2}\right)}{\left[\gamma^{\prime}+\gamma+z^{2} m^{2}+\left(1-z^{2}\right) \kappa^{2}-i \epsilon\right]^{2}}= \\
& =\int_{0}^{\infty} d \gamma^{\prime \prime} \int_{-1}^{1} d \zeta^{\prime} V_{b}\left(\gamma, z ; \gamma^{\prime \prime}, \zeta^{\prime}\right) g_{b}\left(\gamma^{\prime \prime}, \zeta^{\prime} ; \kappa^{2}\right)
\end{aligned}
$$

A direct comparison between Eq. (B1) and Eq. (B2), modulo the value of $\kappa^{2}$, leads to the 
following relation for the interaction kernel $V_{b}^{(L)}$

$$
\begin{aligned}
& V_{b}^{(L)}\left(\gamma, z ; \gamma^{\prime \prime}, \zeta^{\prime}\right)=\lim _{\zeta \rightarrow 0} V_{s}^{(L)}\left(\gamma, z ; \gamma_{i}, z_{i}, \gamma^{\prime \prime}, \zeta, \zeta^{\prime}, \cos \theta\right)=-\frac{g^{2}}{2(4 \pi)^{2}} \times \\
& \lim _{\zeta \rightarrow 0} \int_{-\infty}^{\infty} d \gamma^{\prime} \int_{-1}^{1} d z^{\prime} \frac{1}{\left[\gamma+z^{2} m^{2}+\kappa^{2}\left(1-z^{2}\right)+\gamma^{\prime}+z^{\prime}\left(\frac{M^{2}}{2} z z_{i}+2 \cos \theta \sqrt{\gamma \gamma_{i}}\right)-i \epsilon\right]^{2}} \times \\
& {\left[\frac{(1+z)}{\left(1+\zeta^{\prime}-z_{i} \zeta\right)} \theta\left(\zeta^{\prime}-z-z_{i} \zeta\right) \mathcal{Q}^{\prime}\left(z, z_{i} ; \gamma^{\prime \prime}, \gamma^{\prime}, z^{\prime}, \zeta, \zeta^{\prime}, \mu^{2}\right)+\right.} \\
& \left.+\frac{(1-z)}{\left(1-\zeta^{\prime}+z_{i} \zeta\right)} \theta\left(z-\zeta^{\prime}+z_{i} \zeta\right) \mathcal{Q}^{\prime}\left(-z,-z_{i} ; \gamma^{\prime \prime}, \gamma^{\prime}, z^{\prime}, \zeta,-\zeta^{\prime}, \mu^{2}\right)\right]
\end{aligned}
$$

where $\mathcal{Q}^{\prime}$ can be found in Eq. (B37).

Indeed the limit on $\zeta$ must be carefully performed, since $\mathcal{Q}^{\prime}$ contains two terms, in the first one there is $\theta(\zeta)$ and in the second one there is $\theta(-\zeta)$. Therefore the limit in the two contributions must be $\zeta \rightarrow 0_{+}$and $\zeta \rightarrow 0_{-}$, respectively. In what follows this is understood. The relevant limit to be investigated is (the one corresponding to $\mathcal{Q}^{\prime}\left(-z,-z_{i} ; \gamma^{\prime \prime}, \gamma^{\prime}, z^{\prime}, \zeta,-\zeta^{\prime}, \mu^{2}\right)$ can be carried out analogously)

$$
\begin{aligned}
& \mathcal{H}_{b}\left(z ; \gamma^{\prime \prime}, \gamma^{\prime}, \zeta^{\prime}, \mu^{2}, \kappa^{2}\right)=\lim _{\zeta \rightarrow 0} \frac{(1+z)}{\left(1+\zeta^{\prime}-z_{i} \zeta\right)} \theta\left(\zeta^{\prime}-z-z_{i} \zeta\right) \times \\
& \int_{-1}^{1} d z^{\prime} \frac{\mathcal{Q}^{\prime}\left(z, z_{i} ; \gamma^{\prime \prime}, \gamma^{\prime}, z^{\prime}, \zeta, \zeta^{\prime}, \mu^{2}\right)}{\left[\gamma+z^{2} m^{2}+\kappa^{2}\left(1-z^{2}\right)+\gamma^{\prime}+z^{\prime}\left(\frac{M^{2}}{2} z z_{i}+2 \cos \theta \sqrt{\gamma \gamma_{i}}\right)-i \epsilon\right]^{2}}= \\
& =\lim _{\zeta \rightarrow 0} \frac{(1+z)}{\left(1+\zeta^{\prime}-z_{i} \zeta\right)} \theta\left(\zeta^{\prime}-z-z_{i} \zeta\right) \times \\
& \int_{-1}^{1} \frac{d z^{\prime}}{z^{\prime}} \frac{\Lambda\left(z, \frac{z^{\prime}}{\zeta}, \zeta, \zeta^{\prime}, \gamma^{\prime \prime}, \gamma^{\prime}, z_{i}, \gamma_{i}, \mu^{2}\right)}{\left[\gamma+z^{2} m^{2}+\kappa^{2}\left(1-z^{2}\right)+\gamma^{\prime \prime}+z^{\prime}\left(\frac{M^{2}}{2} z z_{i}+2 \cos \theta \sqrt{\gamma \gamma_{i}}\right)-i \epsilon\right]^{2}} \times \\
& \theta\left(\frac{1+z}{1+\zeta^{\prime}-z_{i} \zeta}-\frac{z^{\prime}}{\zeta}\right)\left[\theta\left(z^{\prime}\right) \theta(\zeta)-\theta\left(-z^{\prime}\right) \theta(-\zeta)\right]= \\
& =\lim _{\zeta \rightarrow 0} \frac{(1+z)}{\left(1+\zeta^{\prime}-z_{i} \zeta\right)} \theta\left(\zeta^{\prime}-z-z_{i} \zeta\right) \theta(\zeta) \int_{0}^{\frac{1}{\zeta}} \frac{d x}{x} \theta\left(\frac{1+z}{1+\zeta^{\prime}-z_{i} \zeta}-x\right) \times \\
& \frac{\Lambda\left(z, x, \zeta, \zeta^{\prime}, \gamma^{\prime \prime}, \gamma^{\prime}, z_{i}, \gamma_{i}, \mu^{2}\right)}{\left[\gamma+z^{2} m^{2}+\kappa^{2}\left(1-z^{2}\right)+\gamma^{\prime}+x \zeta\left(\frac{M^{2}}{2} z z_{i}+2 \cos \theta \sqrt{\gamma \gamma_{i}}\right)-i \epsilon\right]^{2}}+ \\
& -\lim _{\zeta \rightarrow 0} \frac{(1+z)}{\left(1+\zeta^{\prime}-z_{i} \zeta\right)} \theta\left(\zeta^{\prime}-z-z_{i} \zeta\right) \theta(-\zeta) \int_{-\frac{1}{\zeta}}^{0} \frac{d x}{x} \theta\left(\frac{1+z}{1+\zeta^{\prime}-z_{i} \zeta}-x\right) \times \\
& \frac{\Lambda\left(z, x, \zeta, \zeta^{\prime}, \gamma^{\prime \prime}, \gamma^{\prime}, z_{i}, \gamma_{i}, \mu^{2}\right)}{\left[\gamma+z^{2} m^{2}+\kappa^{2}\left(1-z^{2}\right)+\gamma^{\prime}+x \zeta\left(\frac{M^{2}}{2} z z_{i}+2 \cos \theta \sqrt{\gamma \gamma_{i}}\right)-i \epsilon\right]^{2}}
\end{aligned}
$$

where the change of variable $x=z^{\prime} / \zeta$ has been inserted. By recalling that

$$
\frac{1}{|\zeta|} \geq \frac{1+z}{1+\zeta^{\prime}-z_{i} \zeta}
$$


since $1 \geq|\zeta|$ and $\theta\left(\zeta^{\prime}-z_{i} \zeta-z\right)$ one can write

$$
\begin{aligned}
& \mathcal{H}_{b}\left(z ; \gamma^{\prime \prime}, \gamma^{\prime}, \zeta^{\prime}, \mu^{2}, \kappa^{2}\right)=\lim _{\zeta \rightarrow 0} \frac{(1+z)}{\left(1+\zeta^{\prime}-z_{i} \zeta\right)} \theta\left(\zeta^{\prime}-z-z_{i} \zeta\right)[\theta(\zeta)+\theta(-\zeta)] \times \\
& \int_{0}^{\frac{1+z}{1+\zeta^{\prime}-z_{i} \zeta}} \frac{d x}{x} \frac{\Lambda\left(z, x, \zeta, \zeta^{\prime}, \gamma^{\prime \prime}, \gamma^{\prime}, z_{i}, \gamma_{i}, \mu^{2}\right)}{\left[\gamma+z^{2} m^{2}+\kappa^{2}\left(1-z^{2}\right)+\gamma^{\prime}+x \zeta\left(\frac{M^{2}}{2} z z_{i}+2 \cos \theta \sqrt{\gamma \gamma_{i}}\right)-i \epsilon\right]^{2}}
\end{aligned}
$$

where the following identity has been exploited

$$
-\theta(-\zeta) \int_{-\frac{1}{\zeta}}^{0} \frac{d x}{x}=\theta(-\zeta) \int_{0}^{\frac{1}{|\zeta|}} \frac{d x}{x}
$$

Then, one gets

$$
\begin{aligned}
& \mathcal{H}_{b}\left(z ; \gamma^{\prime \prime}, \gamma^{\prime}, \zeta^{\prime}, \mu^{2}, \kappa^{2}\right)=\frac{(1+z)}{\left(1+\zeta^{\prime}\right)} \frac{\theta\left(\zeta^{\prime}-z\right)}{\left[\gamma+z^{2} m^{2}+\kappa^{2}\left(1-z^{2}\right)+\gamma^{\prime}-i \epsilon\right]^{2}} \times \\
& \int_{0}^{\frac{(1+z)}{\left(1+\zeta^{\prime}\right)}} \frac{d x}{x} \tilde{\Lambda}\left(z, x, \zeta^{\prime}, \gamma^{\prime \prime}, \gamma^{\prime}, \mu^{2}, \kappa^{2}\right)
\end{aligned}
$$

where

$$
\begin{aligned}
& \tilde{\Lambda}\left(z, x, \zeta^{\prime}, \gamma^{\prime \prime}, \gamma^{\prime}, \mu^{2}, \kappa^{2}\right)=\sum_{i= \pm} \frac{\partial}{\partial \lambda} \tilde{y}_{i}(0) \frac{1}{\left|\tilde{y}_{i}^{2}(0) \mathcal{A}_{b}\left(\zeta^{\prime}, \gamma^{\prime \prime}, \kappa^{2}\right)-\mu^{2}\right|} \times \\
& {\left[\delta\left(\tilde{y}_{i}(0)\right) y_{i}^{2}(0)-\theta\left(\tilde{y}_{i}(0)\right) \frac{2 \mu^{2} \tilde{y}_{i}(0)}{\tilde{y}_{i}^{2}(0) \mathcal{A}_{b}\left(\zeta^{\prime}, \gamma^{\prime \prime}, \kappa^{2}\right)-\mu^{2}}\right]}
\end{aligned}
$$

with

$$
\begin{aligned}
& \tilde{y}_{ \pm}(0)=\frac{1}{2 \mathcal{A}_{b}\left(\zeta^{\prime}, \gamma^{\prime \prime}, \kappa^{2}\right)} \times \\
& {\left[-\mathcal{B}_{b}\left(z, x, \zeta^{\prime}, \gamma^{\prime \prime}, \gamma^{\prime}, \mu^{2}, 0\right) \pm \sqrt{\mathcal{B}_{b}^{2}\left(z, z^{\prime}, z_{i}, \zeta, \zeta^{\prime}, \gamma^{\prime \prime}, \gamma^{\prime}, \mu^{2}, 0\right)-4 \mu^{2} \mathcal{A}_{b}\left(\zeta^{\prime}, \gamma^{\prime \prime}, \kappa^{2}\right)}\right]} \\
& \frac{\partial}{\partial \lambda} \tilde{y}_{i}(0)=\mp \frac{\left(1+\zeta^{\prime}\right)}{(1+z)} \frac{\tilde{y}_{ \pm}(0)}{\sqrt{\mathcal{B}_{b}^{2}\left(z, x, \zeta^{\prime}, \gamma^{\prime \prime}, \gamma^{\prime}, \mu^{2}, 0\right)-4 \mu^{2} \mathcal{A}_{b}\left(\zeta^{\prime}, \gamma^{\prime \prime}, \kappa^{2}\right)}}
\end{aligned}
$$

and

$$
\begin{aligned}
& \mathcal{A}_{b}\left(\zeta^{\prime}, \gamma^{\prime \prime}, \kappa^{2}\right)=\zeta^{\prime 2} \frac{M^{2}}{4}+\kappa^{2}+\gamma^{\prime \prime} \\
& \mathcal{B}_{b}\left(z, x, \zeta^{\prime}, \gamma^{\prime \prime}, \gamma^{\prime}, \mu^{2}, 0\right)=\mu^{2}+\gamma^{\prime \prime}-\frac{\gamma^{\prime}}{x}
\end{aligned}
$$

The positivity of $\gamma^{\prime \prime}$ in Eq. (122) leads to the positivity of $\mathcal{A}_{b}$. This implies that $\mathcal{B}_{b}$ be negative (cf the end of Appendix [B] ), and eventually $\gamma^{\prime}$ be positive, consistently with Eq. (122), analyzed in Ref. [11]. This could also be expected on physical grounds, since the 
particle production, or a cut in the mathematical language, must be avoided in the bound state kernel $V_{b}^{(L)}\left(\gamma, z ; \gamma^{\prime \prime}, \zeta^{\prime}\right)$, namely in the denominator appearing in Eq. (132). Therefore the interaction kernel $V_{b}^{(L)}$ becomes

$$
\begin{aligned}
& V_{b}^{(L)}\left(\gamma, z ; \gamma^{\prime \prime}, \zeta^{\prime}\right)=-\frac{g^{2}}{2(4 \pi)^{2}} \int_{0}^{\infty} d \gamma^{\prime} \frac{1}{\left[\gamma+z^{2} m^{2}+\kappa^{2}\left(1-z^{2}\right)+\gamma^{\prime}-i \epsilon\right]^{2}} \times \\
& \left\{\frac{(1+z)}{\left(1+\zeta^{\prime}\right)} \theta\left(\zeta^{\prime}-z\right) \int_{0}^{\frac{1+z}{1+\zeta^{\prime}}} \frac{d x}{x} \tilde{\Lambda}\left(z, x, \zeta^{\prime}, \gamma^{\prime \prime}, \gamma^{\prime}, \mu^{2}, \kappa^{2}\right)+\right. \\
& \left.+\frac{(1-z)}{\left(1-\zeta^{\prime}\right)} \theta\left(z-\zeta^{\prime}\right) \int_{0}^{\frac{1-z}{1-\zeta^{\prime}}} \frac{d x}{x} \tilde{\Lambda}\left(-z, x,-\zeta^{\prime}, \gamma^{\prime \prime}, \gamma^{\prime}, \mu^{2}, \kappa^{2}\right)\right\}
\end{aligned}
$$

The new form of the integral integration for $g_{b}\left(\gamma^{\prime}, z ; \kappa^{2}\right)$ is given by

$$
\begin{aligned}
& g_{b}\left(\gamma^{\prime}, z ; \kappa^{2}\right)=-\frac{g^{2}}{2(4 \pi)^{2}} \int_{0}^{\infty} d \gamma^{\prime \prime} \int_{-1}^{1} d \zeta^{\prime} g_{b}\left(\gamma^{\prime \prime}, \zeta^{\prime} ; \kappa^{2}\right) \times \\
& \times \\
& \left\{\frac{(1+z)}{\left(1+\zeta^{\prime}\right)} \theta\left(\zeta^{\prime}-z\right) \int_{0}^{\frac{1+z}{1+\zeta^{\prime}}} \frac{d x}{x} \tilde{\Lambda}\left(z, x, \zeta^{\prime}, \gamma^{\prime \prime}, \gamma^{\prime}, \mu^{2}, \kappa^{2}\right)+\right. \\
& \left.+\frac{(1-z)}{\left(1-\zeta^{\prime}\right)} \theta\left(z-\zeta^{\prime}\right) \int_{0}^{\frac{1-z}{1-\zeta^{\prime}}} \frac{d x}{x} \tilde{\Lambda}\left(-z, x,-\zeta^{\prime}, \gamma^{\prime \prime}, \gamma^{\prime}, \mu^{2}, \kappa^{2}\right)\right\}
\end{aligned}
$$

\section{CONCLUSION}

We analyzed the Bethe-Salpeter equation for scattering states by exploiting the Nakanishi perturbation theory integral representation of the multi-leg transition amplitudes [10]. In this way, one has the possibility to single out their analytic behavior in Minkowski space and to exactly project (namely explicitly integrating over the variable $k^{-}$) any multi-leg amplitude onto the null plane, so that the relative Light-front time (between free legs) can be eliminated. In this context, we have addressed the BSE for the scattering states, in Minkowski space, extending the work of Ref. [11, 12], where the explicitly-covariant Light-front approach was applied to obtain bound states for a massive two-scalar system, interacting through a massive scalar exchange.

A key ingredient of our work is the one-to-one correspondence between the BS amplitude and the LF valence wave function of the interacting system. Such a relation is implemented through an operator that is able to produce the full complexity of the Fock space on top of the valence component [24 29], and noteworthily it makes feasible the study of the Nakanishi amplitude through the valence component, without loosing any physical content. 
The LF projection technique allows us to write down an exact 3D equation for the valence component from the 4D BSE, both for bound and scattering states. In turn, given the relation between BS amplitude and valence component on one side, and the Nakanishi weight function on the other side, one can determine the last one, through a more simple mathematical treatment, namely by using the $3 \mathrm{D}$ equation for the valence component without facing with the complexity of the 4D Minkowski space (see for the bound case [13]).

In the case of scattering states, it has been necessary to consider the PTIR of the halfoff-shell T-matrix, i.e. a four-leg amplitude (while, for the bound state, it is needed the PTIR of the vertex function [11, 12]). Then, applying the projection method, the fourdimensional inhomogeneous BSE has been exactly reduced to a three-dimensional equation for the valence component, that in turn it has allowed to devise an equation for the Nakanishi weight function (a real function), without angular momentum decomposition. The relevant equation, for a massive two-scalar system, interacting through a massive scalar exchange in the continuum, is Eq. (69), that for the sake of clarity we report in this Conclusion, viz

$$
\begin{aligned}
& \int_{-1}^{1} d z^{\prime} \int_{-\infty}^{\infty} d \gamma^{\prime} \frac{g^{(+)}\left(\gamma^{\prime}, z^{\prime}, z ; \gamma_{i}, z_{i}\right)}{\left[\gamma^{\prime}+\gamma+z^{2} m^{2}+\left(1-z^{2}\right) \kappa^{2}+\frac{M}{2} z z^{\prime}\left(\frac{M}{2} z_{i}+k_{i}^{-}\right)+2 z^{\prime} \cos \theta \sqrt{\gamma \gamma_{i}}-i \epsilon\right]^{2}}= \\
& =\mathcal{I}^{L F}\left(\gamma, z ; \gamma_{i}, z_{i}, \cos \theta\right)+ \\
& +\int_{-\infty}^{\infty} d \gamma^{\prime} \int_{-1}^{1} d \zeta \int_{-1}^{1} d \zeta^{\prime} V_{s}^{L F}\left(\gamma, z ; \gamma_{i}, z_{i}, \gamma^{\prime}, \zeta, \zeta^{\prime}, \cos \theta\right) g^{(+)}\left(\gamma^{\prime}, \zeta, \zeta^{\prime} ; \gamma_{i}, z_{i}\right)
\end{aligned}
$$

where the inhomogeneous term, $\mathcal{I}^{L F}$, and the kernel, $V_{s}^{L F}$, are given in Eqs. (70) and (71), respectively. As a by-product, we have obtained the scattering amplitude in terms of the $g^{(+)}, \mathcal{I}^{L F}$ and $V_{s}^{L F}$. This relation will be useful for phenomenological studies.

The explicit expression of the previous integral equation has been also obtained for the 4D BS kernel in ladder approximation, preparing the matter for forthcoming numerical investigations. In particular, a simpler integral equation for determining the Nakanishi amplitude, in ladder approximation, has been worked out by explicitly applying uniqueness, see Eq. (100). It is worth noting that, in ladder approximation, the validity of the uniqueness in the continuum can be checked by obtaining the Nakanishi weight function, through the two possibilities given by the integral equation in (99) and the one in (100). Moreover, it should be emphasized, from one side, the simplicity and the benefit of the LF method for obtaining the kernel of the integral equation for scattering states, and from the other side the elaborated mathematical steps necessary to explicitly use uniqueness in order to simplify the form of integral equation (99) (see e.g. the work of ref. [13]). 
We explored our formalism in ladder approximation, by investigating two limits: i) the zero energy limit $\kappa^{2} \rightarrow 0$ and ii) the Wick-Cutkosky model in the continuum, that corresponds to an exchanged massless scalar, namely $\mu^{2} \rightarrow 0$, obtaining for this case a separable form for the Nakanishi weight function. The cross-ladder contribution will be considered elsewhere. Moreover, a new form of the ladder kernel for bound states has been provided, allowing a simplified form for the integral equation determining the Nakanishi weight.

Nakanishi conjectured [14] that his approach could be valid beyond the perturbative regime. Indeed, numerical solutions of the bound-state problem have shown that, for truncated kernels, his method can be successfully applied. The investigation of the validity of the Nakanishi approach to non perturbative problems in the continuum, as a reasonable extension of what has been already done for the bound-state poles of the transition matrix, can be performed by solving the integral equations obtained in this work.

Finally, the Nakanishi PTIR, applied to bound and scattering states, opens the possibilities of studying many new issues, since it is not constrained to $3+1$ dimensions or to two interacting particles. For instance, let us mention that it could be applied to three-

interacting bosons (see e.g. [21] for the three-boson BSE and the investigation of the final state interaction in three-body decays of heavy mesons), or to consider the extension to $2+1$ dimensions and fermionic systems (useful, e.g., to treat the Dirac electrons in graphene, for reviews see [42, 43]]).

\section{Acknowledgments}

TF and MV acknowledge the hospitality of INFN Sezione di Roma. TF also thanks the partial financial support from the Conselho Nacional de Desenvolvimento Científico e Tecnolíogico (CNPq), the Fundação de Amparo à Pesquisa do Estado de São Paulo (FAPESP) and the Italian MUR through the PRIN 2008. 


\section{Appendix A: The inhomogeneous term in ladder approximation}

The inhomogeneous term, present in the 3D LF equation for scattering states (cf Eq. (69)), is given by

$$
\begin{aligned}
& \mathcal{I}^{L F}\left(\gamma, z ; \gamma_{i}, z_{i}, \cos \theta\right)=p^{+} \int \frac{d k^{-}}{2 \pi} G_{0}^{(12)}(k, p) i \mathcal{K}\left(k, k_{i}, p\right)= \\
& =i^{2} p^{+} \int \frac{d k^{-}}{2 \pi} \frac{1}{\left[\left(\frac{p}{2}+k\right)^{2}-m^{2}+i \epsilon\right]} \frac{1}{\left[\left(\frac{p}{2}-k\right)^{2}-m^{2}+i \epsilon\right]} i \mathcal{K}\left(k, k_{i}, p\right)
\end{aligned}
$$

Let us calculate the term in ladder approximation. Within such an approximation, the kernel $i \mathcal{K}$ is given by (cf Ref. [11])

$$
i \mathcal{K}^{(L)}\left(k, k_{i}\right)=\frac{i(-i g)^{2}}{\left(k-k_{i}\right)^{2}-\mu^{2}+i \epsilon}
$$

where $\mu$ is the mass of the exchanged scalar $\left(g^{2}=16 \pi m^{2} \alpha\right)$ and the momentum transfer is given by $p_{1}-p_{1 i}=(p / 2)+k-(p / 2)-k_{i}$. Let us recall that $k_{i}=\left(p_{1 i}-p_{2 i}\right) / 2$ and $p=p_{1 i}+p_{2 i}=p_{1}+p_{2}$.

Then, in a reference frame where $\mathbf{p}_{\perp}=0$ and $p^{ \pm}=M$, one has

$$
\begin{aligned}
& \mathcal{I}^{(L)}\left(\gamma, z ; \gamma_{i}, z_{i}, \cos \theta\right)=i g^{2} p^{+} \times \\
& \int \frac{d k^{-}}{2 \pi} \frac{1}{\left[\left(\frac{p}{2}+k\right)^{2}-m^{2}+i \epsilon\right]} \frac{1}{\left[\left(\frac{p}{2}-k\right)^{2}-m^{2}+i \epsilon\right]} \frac{1}{\left(k-k_{i}\right)^{2}-\mu^{2}+i \epsilon}= \\
& =i g^{2} p^{+} \int \frac{d k^{-}}{2 \pi} \frac{1}{\left(M / 2+k^{+}\right)\left(M / 2-k^{+}\right)\left(k^{+}-k_{i}^{+}\right)} \times \\
& \frac{1}{\left[\left(\frac{p}{2}+k\right)^{-}-\left(\frac{p}{2}+k\right)_{o n}^{-}+i \epsilon /\left(M / 2+k^{+}\right)\right]} \frac{1}{\left[\left(\frac{p}{2}-k\right)^{-}-\left(\frac{p}{2}-k\right)_{o n}^{-}+i \epsilon /\left(M / 2-k^{+}\right)\right]} \times \\
& \frac{1}{\left(k-k_{i}\right)^{-}-\left(k-k_{i}\right)_{o n}^{-}+i \epsilon /\left(k^{+}-k_{i}^{+}\right)}= \\
& =-i g^{2} p^{+} \int \frac{d k^{-}}{2 \pi} \frac{8}{M^{3}\left(1-z^{2}\right)\left(z-z_{i}\right)} \times \\
& \frac{1}{\frac{M}{2}+k^{-}-\left(\frac{p}{2}+k\right)_{o n}^{-}+i 2 \epsilon /[M /(1-z)]} \frac{1}{\frac{M}{2}-k^{-}-\left(\frac{p}{2}-k\right)_{o n}^{-}+i 2 \epsilon /[M(1+z)]} \times \\
& \frac{1}{k^{-}-k_{i}^{-}-\left(k-k_{i}\right)_{o n}^{-}-i 2 \epsilon /\left[M\left(z-z_{i}\right)\right]}
\end{aligned}
$$

with $z=-2 k^{+} / M, z_{i}=-2 k_{i}^{+} / M=2 k_{i}^{-} / M\left(\right.$ since $\left.p \cdot k_{i}=0\right)$ and

$$
\begin{aligned}
& \left(\frac{p}{2}+k\right)_{o n}^{-}=p_{1 o n}^{-}=\frac{2\left(m^{2}+\gamma\right)}{M(1-z)} \\
& \left(\frac{p}{2}-k\right)_{o n}^{-}=p_{2 o n}^{-}=\frac{2\left(m^{2}+\gamma\right)}{M(1+z)} \\
& \left(k-k_{i}\right)_{o n}^{-}=-\frac{2\left(\mu^{2}+\gamma+\gamma_{i}-2 \cos \theta \sqrt{\left.\gamma \gamma_{i}\right)}\right.}{M\left(z-z_{i}\right)}
\end{aligned}
$$


where $\cos \theta=\widehat{\mathbf{k}}_{\perp} \cdot \widehat{\mathbf{k}}_{i \perp}$, since we have chosen $\widehat{x}=\widehat{\mathbf{k}}_{i \perp}, \gamma=k_{\perp}^{2}$ and $\gamma_{i}=k_{i \perp}^{2}$. It should be pointed out that $1 \geq|z|$ and $1 \geq\left|z_{i}\right|$ since we are considering only particles, i.e $\left(p^{+} / 2\right) \pm k^{+} \geq$ 0 and $\left(p^{+} / 2\right) \pm k_{i}^{+} \geq 0$.

In order to perform the analytic integration, one has to consider the following three poles

$$
\begin{aligned}
& k_{L}^{-}=\frac{2\left(m^{2}+\gamma\right)}{M(1-z)}-\frac{M}{2}-i \frac{2 \epsilon}{M(1-z)} \\
& k_{U}^{-}=-\frac{2\left(m^{2}+\gamma\right)}{M(1+z)}+\frac{M}{2}+i \frac{2 \epsilon}{M(1+z)} \\
& k_{L U}^{-}=k_{i}^{-}-\frac{2\left(\gamma+\gamma_{i}+\mu^{2}-2 \cos \theta \sqrt{\gamma \gamma_{i}}\right)}{M\left(z-z_{i}\right)}+i \frac{2 \epsilon}{M\left(z-z_{i}\right)}= \\
& =\frac{M}{2} z_{i}-\frac{2\left(\gamma+\gamma_{i}+\mu^{2}-2 \cos \theta \sqrt{\gamma \gamma_{i}}\right)}{M\left(z-z_{i}\right)}+i \frac{2 \epsilon}{M\left(z-z_{i}\right)}
\end{aligned}
$$

First of all, let us mention that the cases $|z|=1$ and $\left|z_{i}\right|=1$ lead to a vanishing contributions, as one can check by properly recombining the factors $1 /\left(1-z^{2}\right)$ and $1 /\left(z-z_{i}\right)$ in the integral (A3) and the previous poles. When $1>z>z_{i}>-1$ one can close the integration contour in the lower plane, taking the residue at $k^{-}=k_{L}^{-}$. While for $1>z_{i}>z>-1$ one can choose $k^{-}=k_{U}^{-}$. Then, one has

$$
\begin{aligned}
& \mathcal{I}^{(L)}\left(\gamma, z ; \gamma_{i}, z_{i}, \cos \theta\right)=i g^{2} \int \frac{d k^{-}}{2 \pi} \frac{8}{M^{2}\left(1-z^{2}\right)\left(z-z_{i}\right)} \times \\
& \frac{1}{\left(k^{-}-k_{L}^{-}\right)} \frac{1}{\left(k^{-}-k_{U}^{-}\right)} \frac{1}{\left(k^{-}-k_{L U}\right)}= \\
& =i^{2} g^{2} \frac{8}{M^{2}\left(1-z^{2}\right)\left(z-z_{i}\right)}\left\{-\frac{\theta\left(z-z_{i}\right)}{\frac{2\left(m^{2}+\gamma\right)}{M(1-z)}-\frac{M}{2}+\frac{2\left(m^{2}+\gamma\right)}{M(1+z)}-\frac{M}{2}-i \epsilon} \times\right. \\
& \frac{1}{\frac{2\left(m^{2}+\gamma\right)}{M(1-z)}-\frac{M}{2}\left(1+z_{i}\right)+\frac{2\left(\gamma+\gamma_{i}+\mu^{2}-2 \cos \theta \sqrt{\gamma \gamma_{i}}\right)}{M\left(z-z_{i}\right)}-i \epsilon}+ \\
& +\frac{\theta\left(z_{i}-z\right)}{-\frac{2\left(m^{2}+\gamma\right)}{M(1+z)}+\frac{M}{2}-\frac{2\left(m^{2}+\gamma\right)}{M(1-z)}+\frac{M}{2}+i \epsilon} \times \\
& \left.\frac{1}{-\frac{2\left(m^{2}+\gamma\right)}{M(1+z)}+\frac{M}{2}\left(1-z_{i}\right)+\frac{2\left(\gamma+\gamma_{i}+\mu^{2}-2 \cos \theta \sqrt{\gamma \gamma_{i}}\right)}{M\left(z-z_{i}\right)}+i \epsilon}\right\}
\end{aligned}
$$


Finally, one can rewrite

$$
\begin{aligned}
& \mathcal{I}^{(L)}\left(\gamma, z ; \gamma_{i}, z_{i}, \cos \theta\right)=-g^{2} \frac{8}{M^{2}\left(1-z^{2}\right)\left(z-z_{i}\right)} \frac{1}{M-\frac{2\left(m^{2}+\gamma\right)}{M(1+z)}-\frac{2\left(m^{2}+\gamma\right)}{M(1-z)}+i \epsilon} \times \\
& \left\{\frac{\theta\left(z-z_{i}\right)}{\frac{2\left(m^{2}+\gamma\right)}{M(1-z)}-\frac{M}{2}\left(1+z_{i}\right)+\frac{2\left(\gamma+\gamma_{i}+\mu^{2}-2 \cos \theta \sqrt{\gamma \gamma_{i}}\right)}{M\left(z-z_{i}\right)}-i \epsilon}+\right. \\
& \left.+\frac{\theta\left(z_{i}-z\right)}{-\frac{2\left(m^{2}+\gamma\right)}{M(1+z)}+\frac{M}{2}\left(1-z_{i}\right)+\frac{2\left(\gamma+\gamma_{i}+\mu^{2}-2 \cos \theta \sqrt{\gamma \gamma_{i}}\right)}{M\left(z-z_{i}\right)}+i \epsilon}\right\}= \\
& =g^{2} \frac{1}{\gamma+\left(1-z^{2}\right) \kappa^{2}+z^{2} m^{2}-i \epsilon} \frac{1}{\left(z-z_{i}\right)} \times \\
& \left\{\frac{\theta\left(z-z_{i}\right)}{\frac{\left(m^{2}+\gamma\right)}{(1-z)}-\frac{M^{2}}{4}\left(1+z_{i}\right)+\frac{\left(\gamma+\gamma_{i}+\mu^{2}-2 \cos \theta \sqrt{\gamma \gamma_{i}}\right)}{\left(z-z_{i}\right)}-i \epsilon}+\right. \\
& \left.+\frac{\theta\left(z_{i}-z\right)}{-\frac{\left(m^{2}+\gamma\right)}{(1+z)}+\frac{M^{2}}{4}\left(1-z_{i}\right)+\frac{\left(\gamma+\gamma_{i}+\mu^{2}-2 \cos \theta \sqrt{\gamma \gamma_{i}}\right)}{\left(z-z_{i}\right)}+i \epsilon}\right\}= \\
& =g^{2} \frac{1}{\gamma+\left(1-z^{2}\right) \kappa^{2}+z^{2} m^{2}-i \epsilon} \mathcal{G}^{(L)}\left(\gamma, z ; \gamma_{i}, z_{i}, \cos \theta\right)
\end{aligned}
$$

where $\mathcal{G}^{(L)}\left(\gamma, z ; \gamma_{i}, z_{i}, \cos \theta\right)$ is given by

$$
\begin{aligned}
& \mathcal{G}^{(L)}\left(\gamma, z ; \gamma_{i}, z_{i}, \cos \theta\right)=\frac{1}{\left(z-z_{i}\right)}\left\{\frac{\theta\left(z-z_{i}\right)}{\frac{\left(m^{2}+\gamma\right)}{(1-z)}-\frac{M^{2}}{4}\left(1+z_{i}\right)+\frac{\left(\gamma+\gamma_{i}+\mu^{2}-2 \cos \theta \sqrt{\gamma \gamma_{i}}\right)}{\left(z-z_{i}\right)}-i \epsilon}+\right. \\
& \left.+\frac{\theta\left(z_{i}-z\right)}{-\frac{\left(m^{2}+\gamma\right)}{(1+z)}+\frac{M^{2}}{4}\left(1-z_{i}\right)+\frac{\left(\gamma+\gamma_{i}+\mu^{2}-2 \cos \theta \sqrt{\gamma \gamma_{i}}\right)}{\left(z-z_{i}\right)}+i \epsilon}\right\}= \\
& =\frac{\theta\left(z-z_{i}\right)(1-z)}{\left(z-z_{i}\right)\left[m^{2}+\gamma-\frac{M^{2}}{4}\left(1+z_{i}\right)(1-z)\right]+(1-z)\left(\gamma+\gamma_{i}+\mu^{2}-2 \cos \theta \sqrt{\gamma \gamma_{i}}\right)-i \epsilon}+ \\
& +\frac{\theta\left(z_{i}-z\right)(1+z)}{\left(z-z_{i}\right)\left[\frac{M^{2}}{4}\left(1-z_{i}\right)(1+z)-\left(m^{2}+\gamma\right)\right]+(1+z)\left(\gamma+\gamma_{i}+\mu^{2}-2 \cos \theta \sqrt{\gamma \gamma_{i}}\right)-i \epsilon}= \\
& =\frac{\theta\left(z-z_{i}\right)(1-z)}{\beta\left(z, z_{i}\right)+\gamma\left(1-z_{i}\right)-2(1-z) \cos \theta \sqrt{\gamma \gamma_{i}}-i \epsilon}+ \\
& +\frac{\theta\left(z_{i}-z\right)(1+z)}{\beta\left(-z,-z_{i}\right)+\gamma\left(1+z_{i}\right)-2(1+z) \cos \theta \sqrt{\gamma \gamma_{i}}-i \epsilon}
\end{aligned}
$$

where $\beta\left(z, z_{i}\right)$ is given by:

$$
\begin{aligned}
& \beta\left(z, z_{i}\right)=(1-z)\left[\gamma_{i}+\mu^{2}-\left(z-z_{i}\right)\left(1+z_{i}\right) \frac{M^{2}}{4}-m^{2}\right]+\left(1-z_{i}\right) m^{2}= \\
& =(1-z)\left[\mu^{2}+\frac{M^{2}}{4}(1-z)\left(1+z_{i}\right)-2 m^{2}\right]+\left(1-z_{i}\right) m^{2}
\end{aligned}
$$


where $M^{2} / 4=\left(m^{2}+\gamma_{i}\right) /\left(1-z_{i}^{2}\right)$ has been used. For further purposes, the denominator can written as

$$
\begin{aligned}
& \beta\left(z, z_{i}\right)+\gamma\left(1-z_{i}\right)= \\
& =\left(1-z_{i}\right)\left[\gamma+\left(1-z^{2}\right) \kappa^{2}+z^{2} m^{2}\right]+\beta\left(z, z_{i}\right)-\left(1-z_{i}\right)\left[\left(1-z^{2}\right) \kappa^{2}+z^{2} m^{2}\right]= \\
& =\left(1-z_{i}\right) A+(1-z)\left[\mu^{2}+\frac{M^{2}}{4}(1-z)\left(1+z_{i}\right)-2 m^{2}\right]+\left(1-z_{i}\right)\left(1-z^{2}\right) \frac{M^{2}}{4}= \\
& =\left(1-z_{i}\right) A+(1-z) B
\end{aligned}
$$

where $A=\gamma+\left(1-z^{2}\right) \kappa^{2}+z^{2} m^{2}$ and $B=\mu^{2}-2 \kappa^{2}-z z_{i} M^{2} / 2$

By using the Feynman trick to reduce $\mathcal{I}^{(L)}\left(\gamma, z ; \gamma_{i}, z_{i}, \cos \theta\right)$ to a single denominator, one has

$$
\begin{aligned}
& \mathcal{I}^{(L)}\left(\gamma, z ; \gamma_{i}, z_{i}, \cos \theta\right)=g^{2} \int_{0}^{1} d \alpha \times \\
& {\left[\frac{\theta\left(z-z_{i}\right)(1-z)}{\left[A \alpha+\left(\left(1-z_{i}\right) A+(1-z) B-2(1-z) \cos \theta \sqrt{\gamma \gamma_{i}}\right)(1-\alpha)-i \epsilon\right]^{2}}+\right.} \\
& \left.+\frac{\theta\left(z_{i}-z\right)(1+z)}{\left[A \alpha+\left(\left(1+z_{i}\right) A+(1+z) B-2(1+z) \cos \theta \sqrt{\gamma \gamma_{i}}\right)(1-\alpha)-i \epsilon\right]^{2}}\right\}= \\
& =g^{2} \int_{0}^{1} \frac{d \alpha}{\left[1-z_{i}(1-\alpha)\right]^{2}} \frac{\theta\left(z-z_{i}\right)(1-z)}{\left[A+\left(B-2 \cos \theta \sqrt{\gamma \gamma_{i}}\right) \frac{(1-z)(1-\alpha)}{\left[1-z_{i}(1-\alpha)\right]}-i \epsilon\right]^{2}}+ \\
& +g^{2} \int_{0}^{1} \frac{d \alpha}{\left[1+z_{i}(1-\alpha)\right]^{2}} \frac{\theta\left(z_{i}-z\right)(1+z)}{\left[A+\left(B-2 \cos \theta \sqrt{\gamma \gamma_{i}}\right) \frac{(1+z)(1-\alpha)}{\left[1+z_{i}(1-\alpha)\right]}-i \epsilon\right]^{2}},
\end{aligned}
$$

where $\left[1 \pm z_{i}(1-\alpha)\right] \geq 0$ since $1>\left|z_{i}\right|\left(\left|z_{i}\right|=1\right.$ can be excluded for physical motivation, since this amounts to an infinite energy for one of the incoming particle). The change of variable in the first integral is

$$
y=\frac{(1-z)(1-\alpha)}{\left[1-z_{i}(1-\alpha)\right]}
$$

and in the second one is

$$
y=\frac{(1+z)(1-\alpha)}{\left[1+z_{i}(1-\alpha)\right]}
$$


Then, one has

$$
\begin{aligned}
& \mathcal{I}^{(L)}\left(\gamma, z ; \gamma_{i}, z_{i}, \cos \theta\right)=g^{2} \int_{0}^{1} d y \frac{\theta\left(z-z_{i}\right) \theta\left[1-z-y\left(1-z_{i}\right)\right]}{\left[A+y B-2 y \cos \theta \sqrt{\gamma \gamma_{i}}-i \epsilon\right]^{2}}+ \\
& +g^{2} \int_{0}^{1} d y \frac{\theta\left(z_{i}-z\right) \theta\left[1+z-y\left(1+z_{i}\right)\right]}{\left[A+y B-2 y \cos \theta \sqrt{\gamma \gamma_{i}}-i \epsilon\right]^{2}}= \\
& =g^{2} \int_{-1}^{1} d y \theta(y)\left\{\frac{\theta\left(z-z_{i}\right) \theta\left[1-z-y\left(1-z_{i}\right)\right]}{\left[A+y B-2 y \cos \theta \sqrt{\gamma \gamma_{i}}-i \epsilon\right]^{2}}+\right. \\
& \left.+\frac{\theta\left(z_{i}-z\right) \theta\left[1+z-y\left(1+z_{i}\right)\right]}{\left[A+y B-2 y \cos \theta \sqrt{\gamma \gamma_{i}}-i \epsilon\right]^{2}}\right\}
\end{aligned}
$$

Let us change $y \rightarrow-y$, then one gets

$$
\begin{aligned}
& \mathcal{I}^{(L)}\left(\gamma, z ; \gamma_{i}, z_{i}, \cos \theta\right)=g^{2} \int_{-1}^{1} d y \theta(-y)\left\{\frac{\theta\left(z-z_{i}\right) \theta\left[1-z+y\left(1-z_{i}\right)\right]}{\left[A-y B+2 y \cos \theta \sqrt{\gamma \gamma_{i}}-i \epsilon\right]^{2}}+\right. \\
& \left.+\frac{\theta\left(z_{i}-z\right) \theta\left[1+z+y\left(1+z_{i}\right)\right]}{\left[A-y B+2 y \cos \theta \sqrt{\gamma \gamma_{i}}-i \epsilon\right]^{2}}\right\}= \\
& =g^{2} \int_{-1}^{1} d y \int_{-\infty}^{\infty} d \gamma^{\prime} \frac{\theta(-y) \delta\left(\gamma^{\prime}-\gamma_{a}(y)\right)}{\left[\gamma^{\prime}+\gamma+\left(1-z^{2}\right) \kappa^{2}+z^{2} m^{2}+y \frac{M^{2}}{2} z z_{i}+2 y \cos \theta \sqrt{\gamma \gamma_{i}}-i \epsilon\right]^{2}} \times \\
& \left\{\theta\left(z-z_{i}\right) \theta\left[1-z+y\left(1-z_{i}\right)\right]+\theta\left(z_{i}-z\right) \theta\left[1+z+y\left(1+z_{i}\right)\right]\right\},
\end{aligned}
$$

where the term $y\left(M^{2} / 2\right) z z_{i}$ has been inserted for obtaining the same denominator in Eq. (89) and

$$
\gamma_{a}(y)=-y B-y \frac{M^{2}}{2} z z_{i}=y\left(2 \kappa^{2}-\mu^{2}\right)
$$

\section{Appendix B: The kernel $V_{s}^{L F}$ in ladder approximation}

In the ladder approximation of the 3D LF equation for bound states (cf Eq. (50) ) it is present the following kernel $V_{b}^{(L)}$

$$
\begin{aligned}
& V_{b}^{(L)}\left(\gamma, z ; \gamma^{\prime}, z^{\prime}\right)=i^{2} g^{2} p^{+} \int_{-\infty}^{\infty} \frac{d k^{-}}{2 \pi} \frac{1}{\left[\left(\frac{p}{2}+k\right)^{2}-m^{2}+i \epsilon\right]} \frac{1}{\left[\left(\frac{p}{2}-k\right)^{2}-m^{2}+i \epsilon\right]} \times \\
& \int \frac{d^{4} k^{\prime \prime}}{(2 \pi)^{4}} \frac{1}{\left[k^{\prime \prime 2}+p \cdot k^{\prime \prime} z^{\prime}-\gamma^{\prime}-\kappa^{2}+i \epsilon\right]^{3}} \frac{1}{\left(k-k^{\prime \prime}\right)^{2}-\mu^{2}+i \epsilon}= \\
& =-g^{2} p^{+} \int \frac{d^{4} k^{\prime \prime}}{(2 \pi)^{4}} \frac{1}{\left[k^{\prime \prime 2}+p \cdot k^{\prime \prime} z^{\prime}-\gamma^{\prime}-\kappa^{2}+i \epsilon\right]^{3}} \times \\
& \int_{-\infty}^{\infty} \frac{d k^{-}}{2 \pi} \frac{1}{\left[\left(\frac{p}{2}+k\right)^{2}-m^{2}+i \epsilon\right]} \frac{1}{\left[\left(\frac{p}{2}-k\right)^{2}-m^{2}+i \epsilon\right]} \frac{1}{\left(k-k^{\prime \prime}\right)^{2}-\mu^{2}+i \epsilon}
\end{aligned}
$$


An explicit expression can be found in Ref. [11]. Another explicit expression can be obtained from $V_{s}^{(L)}$ as given in subsect. $\mathrm{VC}$, and recalling that $V_{b}\left(\gamma, z ; \gamma^{\prime}, \zeta^{\prime}\right)=$ $\lim _{\zeta \rightarrow 0} V_{s}\left(\gamma, z ; \gamma_{i}, z_{i}, \gamma^{\prime}, \zeta, \zeta^{\prime}, \cos \theta\right)$

In this Appendix, it will be illustrated in detail the kernel for the scattering-state equation (cf Eq. (69) ). In this case, the kernel acquaints new dependencies: i) upon another compact variable $z^{\prime \prime}$ and ii) the $\cos \theta=\widehat{\mathbf{k}}_{\perp} \cdot \widehat{\mathbf{k}}_{i \perp}$. Moreover, there is an understood dependence upon $\gamma_{i}, z_{i}$ and $p$. In ladder approximation, one has the following expression for $V_{s}^{(L)}$

$$
\begin{aligned}
& V_{s}^{(L)}\left(\gamma, z ; \gamma_{i}, z_{i}, \gamma^{\prime}, \zeta, \zeta^{\prime}, \cos \theta\right)=-p^{+} \int_{-\infty}^{\infty} \frac{d k^{-}}{2 \pi} G_{0}^{(12)}(k, p) \times \\
& \int \frac{d^{4} k^{\prime \prime}}{(2 \pi)^{4}} \frac{\mathcal{K}^{(L)}\left(k, k^{\prime \prime}, p\right)}{\left[k^{\prime \prime 2}+\frac{p^{2}}{4}-m^{2}+p \cdot k^{\prime \prime} \zeta^{\prime}+2 k^{\prime \prime} \cdot k_{i} \zeta-\gamma^{\prime}+i \epsilon\right]^{3}}= \\
& =-g^{2} p^{+} \int_{-\infty}^{\infty} \frac{d k^{-}}{2 \pi} \frac{1}{\left[\left(\frac{p}{2}+k\right)^{2}-m^{2}+i \epsilon\right]} \frac{1}{\left[\left(\frac{p}{2}-k\right)^{2}-m^{2}+i \epsilon\right]} \times \\
& \int \frac{d^{4} k^{\prime \prime}}{(2 \pi)^{4}} \frac{1}{\left[k^{\prime \prime 2}+p \cdot k^{\prime \prime} \zeta^{\prime}+2 k^{\prime \prime} \cdot k_{i} \zeta-\gamma^{\prime}-\kappa^{2}+i \epsilon\right]^{3}} \frac{1}{\left(k-k^{\prime \prime}\right)^{2}-\mu^{2}+i \epsilon}= \\
& =-g^{2} p^{+} \int_{-\infty}^{\infty} \frac{d k^{-}}{2 \pi} \frac{1}{\left[\left(\frac{p}{2}+k\right)^{2}-m^{2}+i \epsilon\right]} \frac{\left.1\left(\frac{p}{2}-k\right)^{2}-m^{2}+i \epsilon\right]}{} \times \\
& \mathcal{P}\left(k, \gamma^{\prime}, \zeta, \zeta^{\prime}, \cos \theta\right)
\end{aligned}
$$

where

$$
\begin{aligned}
& \mathcal{P}\left(k, \gamma^{\prime}, \zeta, \zeta^{\prime}, \cos \theta\right)=\int \frac{d^{4} k^{\prime \prime}}{(2 \pi)^{4}} \frac{1}{\left[k^{\prime \prime 2}+p \cdot k^{\prime \prime} \zeta^{\prime}+2 k^{\prime \prime} \cdot k_{i} \zeta-\gamma^{\prime}-\kappa^{2}+i \epsilon\right]^{3}} \times \\
& \frac{1}{\left(k-k^{\prime \prime}\right)^{2}-\mu^{2}+i \epsilon}
\end{aligned}
$$

Adopting the formula (see also Ref. [11])

$$
\frac{1}{a b^{3}}=\int_{0}^{1} \frac{3 v^{2} d v}{[a(1-v)+b v]^{4}}
$$

where

$$
\begin{aligned}
& a=\left(k-k^{\prime \prime}\right)^{2}-\mu^{2}+i \epsilon \\
& b=k^{\prime \prime 2}+p \cdot k^{\prime \prime} \zeta^{\prime}+2 k^{\prime \prime} \cdot k_{i} \zeta-\gamma^{\prime}-\kappa^{2}+i \epsilon \\
& (1-v) a+v b=(1-v)\left[\left(k-k^{\prime \prime}\right)^{2}-\mu^{2}\right]+v\left[k^{\prime \prime 2}+p \cdot k^{\prime \prime} \zeta^{\prime}+2 k^{\prime \prime} \cdot k_{i} \zeta-\gamma^{\prime}-\kappa^{2}\right]= \\
& =k^{\prime \prime 2}-2 k^{\prime \prime} \cdot\left[(1-v) k-v \zeta^{\prime} \frac{p}{2}-v k_{i} \zeta\right]+(1-v)\left(k^{2}-\mu^{2}\right)-v\left(\gamma^{\prime}+\kappa^{2}\right)+i \epsilon= \\
& =q^{2}-\left[(1-v) k-v \zeta^{\prime} \frac{p}{2}-v k_{i} \zeta\right]^{2}+(1-v)\left(k^{2}-\mu^{2}\right)-v\left(\gamma^{\prime}+\kappa^{2}\right)+i \epsilon
\end{aligned}
$$


with

$$
q=k^{\prime \prime}-(1-v) k+v \zeta^{\prime} \frac{p}{2}+v k_{i} \zeta
$$

Then one has

$$
\begin{aligned}
& \mathcal{P}\left(k, \gamma^{\prime}, \zeta, \zeta^{\prime}, \cos \theta\right)=\frac{1}{(2 \pi)^{4}} \int_{0}^{1} 3 v^{2} d v \int \frac{d^{4} q}{\left[q^{2}+A\left(k, \zeta, \zeta^{\prime}, \cos \theta\right)+i \epsilon\right]^{4}} \\
& =\frac{i}{2(4 \pi)^{2}} \int_{0}^{1} \frac{v^{2} d v}{\left[A\left(k, \zeta, \zeta^{\prime}, \cos \theta\right)+i \epsilon\right]^{2}}
\end{aligned}
$$

where

$$
\begin{aligned}
& A\left(k, \zeta, \zeta^{\prime}, \cos \theta\right)=-\left[(1-v) k-v \zeta^{\prime} \frac{p}{2}-v k_{i} \zeta\right]^{2}+(1-v)\left(k^{2}-\mu^{2}\right)-v\left(\gamma^{\prime}+\kappa^{2}\right)= \\
& =k^{2} v(1-v)+2(1-v) v k \cdot\left[\zeta^{\prime} \frac{p}{2}+k_{i} \zeta\right]-v^{2}\left[\zeta^{\prime} \frac{p}{2}+k_{i} \zeta\right]^{2}-(1-v) \mu^{2}-v\left(\gamma^{\prime}+\kappa^{2}\right)= \\
& =v(1-v)\left[k^{-}\left(k^{+}+\zeta^{\prime} \frac{M}{2}-z_{i} \zeta \frac{M}{2}\right)-\frac{M^{2}}{4} z \zeta^{\prime}-z \zeta \frac{M}{2} k_{i}^{-}-\gamma-2 \zeta \cos \theta \sqrt{\gamma \gamma_{i}}\right]+ \\
& -v^{2}\left[\zeta^{\prime} \frac{p}{2}+k_{i} \zeta\right]^{2}-(1-v) \mu^{2}-v\left(\gamma^{\prime}+\kappa^{2}\right)= \\
& =v(1-v) k^{-}\left(k^{+}+\zeta^{\prime} \frac{M}{2}-z_{i} \zeta \frac{M}{2}\right)-\ell_{D}\left(v, z, \gamma^{\prime}, \zeta, \zeta^{\prime}, \cos \theta\right)
\end{aligned}
$$

with (dependence upon $\gamma_{i}, z_{i}$ and $\mu$ are understood)

$$
\begin{aligned}
& \ell_{D}\left(v, z, \gamma^{\prime}, \zeta, \zeta^{\prime}, \cos \theta\right)=v(1-v)\left[\frac{M^{2}}{4} z \zeta^{\prime}+z \zeta \frac{M}{2} k_{i}^{-}+\gamma+2 \zeta \cos \theta \sqrt{\gamma \gamma_{i}}\right]+v^{2}\left[\zeta^{\prime} \frac{p}{2}+k_{i} \zeta\right]^{2}+ \\
& +(1-v) \mu^{2}+v\left(\gamma^{\prime}+\kappa^{2}\right)= \\
& =v(1-v)\left[\frac{M^{2}}{4} z \zeta^{\prime}+\gamma\right]+v^{2}\left(\zeta^{\prime 2} \frac{M^{2}}{4}+\kappa^{2} \zeta^{2}\right)+ \\
& +(1-v) \mu^{2}+v\left(\gamma^{\prime}+\kappa^{2}\right)+v(1-v) \zeta\left[z z_{i} \frac{M^{2}}{4}+2 \cos \theta \sqrt{\gamma \gamma_{i}}\right]= \\
& =v(1-v)\left[\frac{M^{2}}{4} z \zeta^{\prime}+\gamma+\kappa^{2}\right]+v^{2}\left[\zeta^{\prime 2} \frac{M^{2}}{4}+\kappa^{2}\left(1+\zeta^{2}\right)\right]+ \\
& +(1-v) \mu^{2}+v \gamma^{\prime}+v(1-v) \zeta\left[z z_{i} \frac{M^{2}}{4}+2 \cos \theta \sqrt{\gamma \gamma_{i}}\right]
\end{aligned}
$$

Let us recall that $p \cdot k_{i}=0$ leads to $k_{i}^{+}+k_{i}^{-}=0$ and $\gamma_{i}=M^{2}\left(1-z_{i}^{2}\right) / 4-m^{2}$ as mentioned 
in the previous Appendix. Then the kernel for obtaining the scattering state becomes

$$
\begin{aligned}
& V_{s}^{(L)}\left(\gamma, z ; \gamma_{i}, z_{i}, \gamma^{\prime}, \zeta, \zeta^{\prime}, \cos \theta\right)= \\
& =-g^{2} p^{+} \frac{i}{2(4 \pi)^{2}} \int_{-\infty}^{\infty} \frac{d k^{-}}{2 \pi} \frac{1}{\left[\left(\frac{p}{2}+k\right)^{2}-m^{2}+i \epsilon\right]} \frac{1}{\left[\left(\frac{p}{2}-k\right)^{2}-m^{2}+i \epsilon\right]} \times \\
& \int_{0}^{1} \frac{v^{2} d v}{\left[A\left(p, k, \zeta, \zeta^{\prime}, \cos \theta\right)+i \epsilon\right]^{2}}=-g^{2} p^{+} \frac{i}{2(4 \pi)^{2}} \frac{1}{\left(\frac{M}{2}+k^{+}\right)\left(\frac{M}{2}-k^{+}\right)} \int_{0}^{1} v^{2} d v \int_{-\infty}^{\infty} \frac{d k^{-}}{2 \pi} \times \\
& \frac{1}{\left[\left(\frac{p}{2}+k\right)^{-}-\left(\frac{p}{2}+k\right)_{o n}^{-}+i \epsilon /\left(M / 2+k^{+}\right)\right]} \frac{1}{\left[\left(\frac{p}{2}-k\right)^{-}-\left(\frac{p}{2}-k\right)_{o n}^{-}+i \epsilon /\left(M / 2-k^{+}\right)\right]} \times \\
& \frac{-g^{2} \frac{i}{2 \pi^{2}} \frac{1}{M^{3}\left(1-z^{2}\right)} \int_{0}^{1} v^{2} d v \int_{-\infty}^{\infty} \frac{d k^{-}}{2 \pi} \times}{\frac{1}{\left[\left(\frac{p}{2}+k\right)^{-}-\left(\frac{p}{2}+k\right)_{o n}^{-}+i 2 \epsilon /[M(1-z)]\right.} \frac{1}{\left.\left[\left(\frac{p}{2}-k\right)^{-}-\left(\frac{p}{2}-k\right)_{o n}^{-}+i 2 \epsilon /[M(1+z)]\right)\right]} \times} \\
& \frac{1}{\left\{v(1-v)\left(\zeta^{\prime}-z-z_{i} \zeta\right)\left[k^{-}-k_{D}^{-}+i 2 \epsilon /\left[M v(1-v)\left(\zeta^{\prime}-z-z_{i} \zeta\right)\right]\right]\right\}^{2}}=
\end{aligned}
$$

where

$$
\begin{aligned}
& \left(\frac{p}{2}+k\right)_{o n}^{-}=p_{1 o n}^{-}=\frac{2\left(m^{2}+\gamma\right)}{M(1-z)} \\
& \left(\frac{p}{2}-k\right)_{o n}^{-}=p_{2 o n}^{-}=\frac{2\left(m^{2}+\gamma\right)}{M(1+z)} \\
& k_{D}^{-}=\frac{2 \ell_{D}\left(v, \gamma, z, \gamma^{\prime}, \zeta, \zeta^{\prime}, \cos \theta\right)}{M v(1-v)\left(\zeta^{\prime}-z-z_{i} \zeta\right)}
\end{aligned}
$$

One has the following three poles $(1>z>-1)$

$$
\begin{aligned}
& k_{n}=\left(\frac{p}{2}+k\right)_{o n}^{-}-\frac{M}{2}-i \epsilon \\
& k_{p}=-\left(\frac{p}{2}-k\right)_{o n}^{-}+\frac{M}{2}+i \epsilon \\
& k_{d}=k_{D}^{-}-i \frac{\epsilon}{\left(\zeta^{\prime}-z-z_{i} \zeta\right)}
\end{aligned}
$$


If $\zeta^{\prime}>z+z_{i} \zeta$ one can close in the upper plane taking the residue at $k_{p}$, i.e.

$$
\begin{aligned}
& \int_{-\infty}^{\infty} \frac{d k^{-}}{2 \pi} \frac{1}{\left[\left(\frac{p}{2}+k\right)^{-}-\left(\frac{p}{2}+k\right)_{o n}^{-}+i \epsilon\right]} \frac{1}{\left.\left[\left(\frac{p}{2}-k\right)^{-}-\left(\frac{p}{2}-k\right)_{o n}^{-}+i \epsilon\right)\right]} \times \\
& \frac{1}{\left[v(1-v)\left(\zeta^{\prime}-z-z_{i} \zeta\right)\left(k^{-}-k_{D}^{-}\right)+i \epsilon\right]^{2}}= \\
& =-i \frac{1}{\left[M-\left(\frac{p}{2}-k\right)_{o n}^{-}-\left(\frac{p}{2}+k\right)_{o n}^{-}+i \epsilon\right]} \times \\
& \frac{1}{\left\{v(1-v)\left(\zeta^{\prime}-z-z_{i} \zeta\right)\left[\frac{M}{2}-\left(\frac{p}{2}-k\right)_{o n}^{-}-k_{D}^{-}\right]+i \epsilon\right\}^{2}}= \\
& =-i \frac{M}{\left[M^{2}-4 \frac{\left(m^{2}+\gamma\right)}{\left(1-z^{2}\right)}+i \epsilon\right]} \frac{1}{\left[v(1-v)\left(\zeta^{\prime}-z-z_{i} \zeta\right)\left(\frac{M}{2}-\frac{2}{M} \frac{m^{2}+\gamma}{1+z}-k_{D}^{-}\right)+i \epsilon\right]^{2}}
\end{aligned}
$$

If $z+z_{i} \zeta>\zeta^{\prime}$ one can close in the lower plane taking the residue at $k_{n}$, i.e.

$$
\begin{aligned}
& \int_{-\infty}^{\infty} \frac{d k^{-}}{2 \pi} \frac{1}{\left[\left(\frac{p}{2}+k\right)^{-}-\left(\frac{p}{2}+k\right)_{o n}^{-}+i \epsilon\right]} \frac{1}{\left.\left[\left(\frac{p}{2}-k\right)^{-}-\left(\frac{p}{2}-k\right)_{o n}^{-}+i \epsilon\right)\right]} \times \\
& \frac{1}{\left[v(1-v)\left(\zeta^{\prime}-z-z_{i} \zeta\right)\left(k^{-}-k_{D}^{-}-i \epsilon\right)\right]^{2}}=-i \frac{1}{\left[M-\left(\frac{p}{2}+k\right)_{o n}^{-}-\left(\frac{p}{2}-k\right)_{o n}^{-}+i \epsilon\right]} \times \\
& \frac{1}{\left\{v(1-v)\left(\zeta^{\prime}-z-z_{i} \zeta\right)\left[-\frac{M}{2}+\left(\frac{p}{2}+k\right)_{o n}^{-}-k_{D}^{-}-i \epsilon\right]\right\}^{2}}= \\
& =-i \frac{M}{\left[M^{2}-4 \frac{\left(m^{2}+\gamma\right)}{\left(1-z^{2}\right)}+i \epsilon\right]} \frac{1}{\left[v(1-v)\left(\zeta^{\prime}-z-z_{i} \zeta\right)\left(\frac{M}{2}-\frac{2}{M} \frac{m^{2}+\gamma}{(1-z)}+k_{D}^{-}+i \epsilon\right)\right]^{2}}
\end{aligned}
$$

Then, by exploiting Eq. (B10) one has

$$
\begin{aligned}
& V_{s}^{(L)}\left(\gamma, z ; \gamma_{i}, z_{i}, \gamma^{\prime}, \zeta, \zeta^{\prime}, \cos \theta\right)=-\frac{g^{2}}{2(2 \pi)^{2}\left(1-z^{2}\right)} \frac{1}{\left[M^{2}-4 \frac{\left(m^{2}+\gamma\right)}{\left(1-z^{2}\right)}+i \epsilon\right]} \times \\
& \int_{0}^{1} d v v^{2} \mathcal{F}\left(v, \gamma, z ; \gamma^{\prime}, \zeta, \zeta^{\prime}, \cos \theta\right)= \\
& =\frac{g^{2}}{2(4 \pi)^{2}} \frac{1}{\left[\gamma+\left(1-z^{2}\right) \kappa^{2}+z^{2} m^{2}-i \epsilon\right]} \int_{0}^{1} d v v^{2} \mathcal{F}\left(v, \gamma, z ; \gamma^{\prime}, \zeta, \zeta^{\prime}, \cos \theta\right)
\end{aligned}
$$


where

$$
\begin{aligned}
& \mathcal{F}\left(v, \gamma, z ; \gamma^{\prime}, \zeta, \zeta^{\prime}, \cos \theta\right)= \\
& =\frac{\theta\left(\zeta^{\prime}-z-z_{i} \zeta\right)}{\left[v(1-v)\left(\zeta^{\prime}-z-z_{i} \zeta\right)\left(\frac{M^{2}}{4}-\frac{m^{2}+\gamma}{1+z}\right)-\ell_{D}\left(v, \gamma, z, \gamma^{\prime}, \zeta, \zeta^{\prime}, \cos \theta\right)+i \epsilon\right]^{2}}+ \\
& +\frac{\theta\left(z+z_{i} \zeta-\zeta^{\prime}\right)}{\left[v(1-v)\left(z+z_{i} \zeta-\zeta^{\prime}\right)\left(\frac{M^{2}}{4}-\frac{m^{2}+\gamma}{1-z}\right)-\ell_{D}\left(v, \gamma, z, \gamma^{\prime}, \zeta, \zeta^{\prime}, \cos \theta\right)+i \epsilon\right]^{2}}= \\
& =\frac{(1+z)^{2} \theta\left(\zeta^{\prime}-z-z_{i} \zeta\right)}{\left[\mathcal{D}\left(v, z, z_{i}, \zeta, \zeta^{\prime}\right)+v(1-v)(1+z) \zeta\left(\frac{M^{2}}{2} z z_{i}+2 \cos \theta \sqrt{\gamma \gamma_{i}}\right)-i \epsilon\right]^{2}}+ \\
& +\frac{(1-z)^{2} \theta\left(z+z_{i} \zeta-\zeta^{\prime}\right)}{\left[\mathcal{D}\left(v,-z,-z_{i}, \zeta,-\zeta^{\prime}\right)+v(1-v)(1-z) \zeta\left(\frac{M^{2}}{2} z z_{i}+2 \cos \theta \sqrt{\gamma \gamma_{i}}\right)-i \epsilon\right]^{2}}
\end{aligned}
$$

with

$$
\begin{aligned}
& \mathcal{D}\left(v, z, z_{i}, \zeta, \zeta^{\prime}\right)=v(1-v)\left(\zeta^{\prime}-z-z_{i} \zeta\right)\left(m^{2}+\gamma-(1+z) \frac{M^{2}}{4}\right)+ \\
& +(1+z) \ell_{D}\left(v, \gamma, z, \gamma^{\prime}, \zeta, \zeta^{\prime}, \cos \theta\right)-v(1-v)(1+z) \zeta\left(\frac{M^{2}}{2} z z_{i}+2 \cos \theta \sqrt{\gamma \gamma_{i}}\right)= \\
& =v(1-v)\left[\left(\zeta^{\prime}-z-z_{i} \zeta\right)\left(\kappa^{2}+\gamma\right)+z^{2} \frac{M^{2}}{4}\left(1+\zeta^{\prime}-\zeta z_{i}\right)+(1+z)\left(\gamma+\kappa^{2}\right)\right]+ \\
& +(1+z)\left\{v^{2}\left[\zeta^{\prime 2} \frac{M^{2}}{4}+\kappa^{2}\left(1+\zeta^{2}\right)\right]+(1-v) \mu^{2}+v \gamma^{\prime}\right\}= \\
& =v(1-v)\left[\left(1+\zeta^{\prime}-z_{i} \zeta\right)\left(\kappa^{2}+\gamma\right)+\frac{M^{2}}{4} z^{2}\left(1+\zeta^{\prime}-\zeta z_{i}\right)\right]+ \\
& +(1+z)\left\{v^{2}\left[\zeta^{\prime 2} \frac{M^{2}}{4}+\kappa^{2}\left(1+\zeta^{2}\right)\right]+(1-v) \mu^{2}+v \gamma^{\prime}\right\}= \\
& =X\left(v, z_{i}, \zeta, \zeta^{\prime}\right)\left[\gamma+z^{2} m^{2}+\kappa^{2}\left(1-z^{2}\right)+\Gamma\left(v, z, z_{i}, \zeta, \zeta^{\prime}, \gamma^{\prime}\right)\right]
\end{aligned}
$$

In Eq. (B16), the following notation has been used

$$
\begin{aligned}
& X\left(v, z_{i}, \zeta, \zeta^{\prime}\right)=v(1-v)\left(1+\zeta^{\prime}-z_{i} \zeta\right) \\
& \Gamma\left(v, z, z_{i}, \zeta, \zeta^{\prime}, \gamma^{\prime}\right)=\frac{(1+z)}{\left(1+\zeta^{\prime}-z_{i} \zeta\right)} \times \\
& \left\{\frac{v}{(1-v)}\left[\zeta^{\prime 2} \frac{M^{2}}{4}+\kappa^{2}\left(1+\zeta^{2}\right)+\gamma^{\prime}\right]+\frac{\mu^{2}}{v}+\gamma^{\prime}\right\}
\end{aligned}
$$

Notice that

$$
\frac{3}{4} \geq X\left(v, z_{i}, \zeta, \zeta^{\prime},\right) \geq 0, \quad \frac{3}{4} \geq X\left(v,-z_{i}, \zeta,-\zeta^{\prime},\right) \geq 0
$$

where the positivity follows from the presence of the theta functions in Eq. (B15). 
Then one can rewrite

$$
\begin{aligned}
& \mathcal{F}\left(v, \gamma, z ; \gamma^{\prime}, \zeta, \zeta^{\prime}, \cos \theta\right)=\frac{(1+z)^{2}}{X^{2}\left(v, z_{i}, \zeta, \zeta^{\prime}\right)} \times \\
& \frac{\theta\left(\zeta^{\prime}-z-z_{i} \zeta\right)}{\left[\gamma+z^{2} m^{2}+\kappa^{2}\left(1-z^{2}\right)+\Gamma\left(v, z, z_{i}, \zeta, \zeta^{\prime}, \gamma^{\prime}\right)+Z\left(z, \zeta, \zeta^{\prime} ; z_{i}\right) C-i \epsilon\right]^{2}}+ \\
& +\frac{(1-z)^{2}}{X^{2}\left(v,-z_{i}, \zeta,-\zeta^{\prime}\right)} \times \\
& \frac{\theta\left(z+z_{i} \zeta-\zeta^{\prime}\right)}{\left[\gamma+z^{2} m^{2}+\kappa^{2}\left(1-z^{2}\right)+\Gamma\left(v,-z,-z_{i}, \zeta,-\zeta^{\prime}, \gamma^{\prime}\right)+Z\left(-z, \zeta,-\zeta^{\prime} ;-z_{i}\right) C-i \epsilon\right]^{2}}(
\end{aligned}
$$

where

$$
C=\frac{M^{2}}{2} z z_{i}+2 \cos \theta \sqrt{\gamma \gamma_{i}}
$$

and

$$
Z\left(z, \zeta, \zeta^{\prime} ; z_{i}\right)=\zeta \frac{(1+z)}{\left(1+\zeta^{\prime}-z_{i} \zeta\right)}
$$

Once more, the presence of the theta functions in Eq. (B15) helps to find the following constrain

$$
\left|Z\left(z, \zeta, \zeta^{\prime}, z_{i}\right)\right| \leq|\zeta|, \quad\left|Z\left(-z, \zeta,-\zeta^{\prime},-z_{i}\right)\right| \leq|\zeta|
$$

Notice that one can always put $\epsilon \rightarrow X\left(v, z_{i}, \zeta, \zeta^{\prime}\right) \epsilon$, given the limits on $X\left(v, z_{i}, \zeta, \zeta^{\prime}\right)$.

The kernel $V_{s}^{(L)}$ can be evaluated by slightly elaborating the standard Feynman trick. Indeed one can use

$$
\begin{aligned}
& \frac{1}{B A^{2}}=\lim _{\lambda \rightarrow 0} \frac{1}{\lambda}\left[\frac{1}{B A}-\frac{1}{B(A+\lambda)}\right]= \\
& =\lim _{\lambda \rightarrow 0} \frac{1}{\lambda}\left\{\int_{0}^{1} d \xi \frac{1}{[B-\xi(B-A)]^{2}}-\int_{0}^{1} d \xi \frac{1}{[B-\xi(B-A)+\xi \lambda]^{2}}\right\}
\end{aligned}
$$

By applying the above relation with the following identifications

$$
\begin{aligned}
& A_{ \pm}=\gamma+z^{2} m^{2}+\kappa^{2}\left(1-z^{2}\right)+\Gamma\left(v, z, z_{i}, \zeta, \zeta^{\prime}, \gamma^{\prime}\right)+Z\left(z, \zeta, \zeta^{\prime} ; z_{i}\right) C-i \epsilon \\
& B_{ \pm}=\gamma+z^{2} m^{2}+\kappa^{2}\left(1-z^{2}\right)-i \epsilon
\end{aligned}
$$

one gets

$$
\begin{aligned}
& V_{s}^{(L)}\left(\gamma, z ; \gamma_{i}, z_{i}, \gamma^{\prime}, \zeta, \zeta^{\prime}, \cos \theta\right)=-\frac{g^{2}}{2(4 \pi)^{2}} \times \\
& {\left[\frac{(1+z)}{\left(1+\zeta^{\prime}-z_{i} \zeta\right)} \theta\left(\zeta^{\prime}-z-z_{i} \zeta\right) \mathcal{H}^{\prime}\left(\gamma, z, z_{i} ; \gamma^{\prime}, \zeta, \zeta^{\prime}, \cos \theta, \mu^{2}\right)+\right.} \\
& \left.+\frac{(1-z)}{\left(1-\zeta^{\prime}+z_{i} \zeta\right)} \theta\left(z-\zeta^{\prime}+z_{i} \zeta\right) \mathcal{H}^{\prime}\left(\gamma,-z,-z_{i} ; \gamma^{\prime}, \zeta,-\zeta^{\prime}, \cos \theta, \mu^{2}\right)\right]
\end{aligned}
$$


where

$$
\begin{aligned}
& \mathcal{H}^{\prime}\left(\gamma, z, z_{i} ; \gamma^{\prime}, \zeta, \zeta^{\prime}, \cos \theta, \mu^{2}\right)= \\
& =\lim _{\lambda \rightarrow 0} \frac{1}{\lambda}\left[\mathcal{H}\left(\gamma, z, z_{i} ; \gamma^{\prime}, \zeta, \zeta^{\prime}, \cos \theta, \mu^{2}, \lambda\right)-\mathcal{H}\left(\gamma, z, z_{i} ; \gamma^{\prime}, \zeta, \zeta^{\prime}, \cos \theta, \mu^{2}, 0\right)\right]
\end{aligned}
$$

with

$$
\begin{aligned}
& \mathcal{H}\left(\gamma, z, z_{i} ; \gamma^{\prime}, \zeta, \zeta^{\prime}, \cos \theta, \mu^{2}, \lambda\right)=\frac{(1+z)}{\left(1+\zeta^{\prime}-z_{i} \zeta\right)} \int_{0}^{1} \frac{d v}{(1-v)^{2}} \int_{0}^{1} d \xi \times \\
& \frac{1}{\left\{\gamma+z^{2} m^{2}+\kappa^{2}\left(1-z^{2}\right)+\xi\left[\Gamma\left(v, z, z_{i}, \zeta, \zeta^{\prime}, \gamma^{\prime}\right)+Z\left(z, \zeta, \zeta^{\prime} ; z_{i}\right) C+\lambda\right]-i \epsilon\right\}^{2}}= \\
& =\frac{(1+z)}{\left(1+\zeta^{\prime}-z_{i} \zeta\right)} \int_{0}^{1} \frac{d v}{(1-v)^{2}} \int_{0}^{1} d \xi \int_{-\infty}^{\infty} d \gamma^{\prime \prime} \times \\
& \frac{\delta\left[\gamma^{\prime \prime}-\xi \Gamma\left(v, z, z_{i}, \zeta, \zeta^{\prime}, \gamma^{\prime}\right)-\xi \lambda\right]}{\left[\gamma+z^{2} m^{2}+\kappa^{2}\left(1-z^{2}\right)+\gamma^{\prime \prime}+\xi Z\left(z, \zeta, \zeta^{\prime} ; z_{i}\right) C-i \epsilon\right]^{2}}
\end{aligned}
$$

It should be pointed out that, in order to obtain Eq. (B24), the limit on $\lambda$ has been exchanged with the integral over $v$.

By inserting the following change of variable

$$
z^{\prime}=\xi Z\left(z, \zeta, \zeta^{\prime} ; z_{i}\right)
$$

such that $\left|z^{\prime}\right| \leq|\zeta| \leq 1$ from Eq. (B21), one gets

$$
\begin{aligned}
& \mathcal{H}\left(\gamma, z, z_{i} ; \gamma^{\prime}, \zeta, \zeta^{\prime}, \cos \theta, \mu^{2}, \lambda\right)=\frac{(1+z)}{\left(1+\zeta^{\prime}-z_{i} \zeta\right)} \int_{0}^{1} \frac{d v}{(1-v)^{2}} \int_{-\infty}^{\infty} d \gamma^{\prime \prime} \int_{-1}^{1} \frac{d z^{\prime}}{Z\left(z, \zeta, \zeta^{\prime} ; z_{i}\right)} \times \\
& \frac{\theta\left(z^{\prime}\right) \theta\left(Z\left(z, \zeta, \zeta^{\prime} ; z_{i}\right)-z^{\prime}\right)-\theta\left(-z^{\prime}\right) \theta\left(z^{\prime}-Z\left(z, \zeta, \zeta^{\prime} ; z_{i}\right)\right)}{\left[\gamma+z^{2} m^{2}+\kappa^{2}\left(1-z^{2}\right)+\gamma^{\prime \prime}+z^{\prime} C-i \epsilon\right]^{2}} \delta\left[\gamma^{\prime \prime}-z^{\prime} \frac{\Gamma\left(v, z, z_{i}, \zeta, \zeta^{\prime}, \gamma^{\prime}\right)+\lambda}{Z\left(z, \zeta, \zeta^{\prime} ; z_{i}\right)}\right]= \\
& =\frac{(1+z)}{\left(1+\zeta^{\prime}-z_{i} \zeta\right)} \int_{0}^{1} \frac{d v}{(1-v)^{2}} \int_{-\infty}^{\infty} d \gamma^{\prime \prime} \int_{-1}^{1} d z^{\prime} \frac{\left|Z\left(z, \zeta, \zeta^{\prime} ; z_{i}\right)\right|}{Z\left(z, \zeta, \zeta^{\prime} ; z_{i}\right)} \times \\
& \delta\left[\gamma^{\prime \prime} Z\left(z, \zeta, \zeta^{\prime} ; z_{i}\right)-z^{\prime} \Gamma\left(v, z, z_{i}, \zeta, \zeta^{\prime}, \gamma^{\prime}\right)-z^{\prime} \lambda\right] \times \\
& \frac{\theta\left(z^{\prime}\right) \theta\left(Z\left(z, \zeta, \zeta^{\prime} ; z_{i}\right)-z^{\prime}\right)-\theta\left(-z^{\prime}\right) \theta\left(z^{\prime}-Z\left(z, \zeta, \zeta^{\prime} ; z_{i}\right)\right)}{\left[\gamma+z^{2} m^{2}+\kappa^{2}\left(1-z^{2}\right)+\gamma^{\prime \prime}+z^{\prime} C-i \epsilon\right]^{2}}
\end{aligned}
$$


Finally, let us perform the change $v \rightarrow y / 1+y$, then one has

$$
\begin{aligned}
& \mathcal{H}\left(\gamma, z, z_{i} ; \gamma^{\prime}, \zeta, \zeta^{\prime}, \cos \theta, \mu^{2}, \lambda\right)=\frac{(1+z)}{\left(1+\zeta^{\prime}-z_{i} \zeta\right)} \int_{0}^{\infty} d y \int_{-\infty}^{\infty} d \gamma^{\prime \prime} \times \\
& \int_{-1}^{1} d z^{\prime} \delta\left[\gamma^{\prime \prime} Z\left(z, \zeta, \zeta^{\prime} ; z_{i}\right)-z^{\prime} \tilde{\Gamma}\left(y, z, \zeta, \zeta^{\prime}, \gamma^{\prime}\right)-z^{\prime} \lambda\right] \times \\
& \frac{\theta\left(z^{\prime}\right) \theta\left(Z\left(z, \zeta, \zeta^{\prime} ; z_{i}\right)-z^{\prime}\right)+\theta\left(-z^{\prime}\right) \theta\left(z^{\prime}-Z\left(z, \zeta, \zeta^{\prime} ; z_{i}\right)\right)}{\left[\gamma+z^{2} m^{2}+\kappa^{2}\left(1-z^{2}\right)+\gamma^{\prime \prime}+z^{\prime} C-i \epsilon\right]^{2}}= \\
& =\int_{-\infty}^{\infty} d \gamma^{\prime \prime} \int_{-1}^{1} d z^{\prime} \frac{\theta\left(z^{\prime}\right) \theta\left(Z\left(z, \zeta, \zeta^{\prime} ; z_{i}\right)-z^{\prime}\right)+\theta\left(-z^{\prime}\right) \theta\left(z^{\prime}-Z\left(z, \zeta, \zeta^{\prime} ; z_{i}\right)\right)}{\left[\gamma+z^{2} m^{2}+\kappa^{2}\left(1-z^{2}\right)+\gamma^{\prime \prime}+z^{\prime} C-i \epsilon\right]^{2}} \times \\
& \int_{0}^{\infty} d y \delta\left[\gamma^{\prime \prime} \zeta-z^{\prime} \tilde{\Gamma}\left(y, \zeta, \zeta^{\prime}, \gamma^{\prime}\right)-z^{\prime} \frac{\left(1+\zeta^{\prime}-z_{i} \zeta\right)}{(1+z)}=\right. \\
& =\int_{-\infty}^{\infty} d \gamma^{\prime \prime} \int_{-1}^{1} \frac{d z^{\prime}}{z^{\prime}} \frac{\theta\left(z^{\prime}\right) \theta\left(Z\left(z, \zeta, \zeta^{\prime} ; z_{i}\right)-z^{\prime}\right)-\theta\left(-z^{\prime}\right) \theta\left(z^{\prime}-Z\left(z, \zeta, \zeta^{\prime} ; z_{i}\right)\right)}{\left[\gamma+z^{2} m^{2}+\kappa^{2}\left(1-z^{2}\right)+\gamma^{\prime \prime}+z^{\prime} C-i \epsilon\right]^{2}} \times \\
& \int_{0}^{\infty} d y \delta\left[\frac{1}{y}\left(y^{2} \mathcal{A}+y \mathcal{B}+\mu^{2}\right)\right]
\end{aligned}
$$

where

$$
\tilde{\Gamma}\left(y, \zeta, \zeta^{\prime}, \gamma^{\prime}\right)=y\left[\zeta^{\prime 2} \frac{M^{2}}{4}+\kappa^{2}\left(1+\zeta^{2}\right)\right]+\frac{(1+y)}{y} \mu^{2}+(1+y) \gamma^{\prime}
$$

and the argument of the delta has been recast in the following form

$$
\begin{aligned}
& \tilde{\Gamma}\left(y, \zeta, \zeta^{\prime}, \gamma^{\prime}\right)+\frac{\left(1+\zeta^{\prime}-z_{i} \zeta\right)}{(1+z)} \lambda-\gamma^{\prime \prime} \frac{\zeta}{z^{\prime}}= \\
& =\frac{1}{y}\left[y^{2} \mathcal{A}\left(\zeta, \zeta^{\prime}, \gamma^{\prime}, \kappa^{2}\right)+y \mathcal{B}\left(z, z^{\prime}, z_{i}, \zeta, \zeta^{\prime}, \gamma^{\prime}, \gamma^{\prime \prime}, \mu^{2}, \lambda\right)+\mu^{2}\right]
\end{aligned}
$$

with

$$
\begin{aligned}
& \mathcal{A}\left(\zeta, \zeta^{\prime}, \gamma^{\prime}, \kappa^{2}\right)=\zeta^{\prime 2} \frac{M^{2}}{4}+\kappa^{2}\left(1+\zeta^{2}\right)+\gamma^{\prime} \\
& \mathcal{B}\left(z, z^{\prime}, z_{i}, \zeta, \zeta^{\prime}, \gamma^{\prime}, \gamma^{\prime \prime}, \mu^{2}, \lambda\right)=\mu^{2}+\gamma^{\prime}-\gamma^{\prime \prime} \frac{\zeta}{z^{\prime}}+\lambda \frac{\left(1+\zeta^{\prime}-z_{i} \zeta\right)}{(1+z)}
\end{aligned}
$$

The integral over $y$ is readily done, obtaining

$$
\begin{aligned}
& \mathcal{H}\left(\gamma, z, z_{i} ; \gamma^{\prime}, \zeta, \zeta^{\prime}, \cos \theta, \mu^{2}, \lambda\right)=\int_{-\infty}^{\infty} d \gamma^{\prime \prime} \int_{-1}^{1} \frac{d z^{\prime}}{z^{\prime}} \times \\
& \theta\left(\frac{1+z}{1+\zeta^{\prime}-z_{i} \zeta}-\frac{z^{\prime}}{\zeta}\right) \frac{\theta\left(z^{\prime}\right) \theta(\zeta)-\theta\left(-z^{\prime}\right) \theta(-\zeta)}{\left[\gamma+z^{2} m^{2}+\kappa^{2}\left(1-z^{2}\right)+\gamma^{\prime \prime}+z^{\prime} C-i \epsilon\right]^{2}} \times \\
& \sum_{i= \pm} \theta\left(y_{i}(\lambda)\right) \frac{y_{i}^{2}(\lambda)}{\left|y_{i}^{2}(\lambda) \mathcal{A}\left(\zeta, \zeta^{\prime}, \gamma^{\prime}, \kappa^{2}\right)-\mu^{2}\right|}
\end{aligned}
$$


where we have used Eq. (B20) (exploiting the theta functions for determining the signs of $\zeta$ ) and

$$
\delta(f(y))=\sum_{i} \frac{\delta\left(y-y_{i}\right)}{\left|f^{\prime}\left(y_{i}\right)\right|}
$$

with $f\left(y_{i}\right)=0$ and $f^{\prime}\left(y_{i}\right)$ the derivative evaluated at $y=y_{i}$. Notice that $\theta\left(y_{i}(\lambda)\right)$ comes from the lower extrema in the integral on $y$. Moreover, for simplifying the notation, we have dropped the dependence upon some variables in $y_{ \pm}$, that represent the two solutions of the second order equation

$$
y^{2} \mathcal{A}\left(\zeta, \zeta^{\prime}, \gamma^{\prime}, \kappa^{2}\right)+y \mathcal{B}\left(z, z^{\prime}, z_{i}, \zeta, \zeta^{\prime}, \gamma^{\prime}, \gamma^{\prime \prime}, \mu^{2}, \lambda\right)+\mu^{2}=0
$$

Once $\mathcal{H}\left(\gamma, z, z_{i} ; \gamma^{\prime}, \zeta, \zeta^{\prime}, \cos \theta, \mu^{2}, \lambda\right)$ is formally obtained, its derivative with respect to $\lambda$, at $\lambda=0$, can be evaluated as follows

$$
\begin{aligned}
& \mathcal{H}^{\prime}\left(\gamma, z, z_{i} ; \gamma^{\prime}, \zeta, \zeta^{\prime}, \cos \theta, \mu^{2}\right)=\int_{-\infty}^{\infty} d \gamma^{\prime \prime} \int_{-1}^{1} \frac{d z^{\prime}}{z^{\prime}} \times \\
& \theta\left(\frac{1+z}{1+\zeta^{\prime}-z_{i} \zeta}-\frac{z^{\prime}}{\zeta}\right) \frac{\theta\left(z^{\prime}\right) \theta(\zeta)-\theta\left(-z^{\prime}\right) \theta(-\zeta)}{\left[\gamma+z^{2} m^{2}+\kappa^{2}\left(1-z^{2}\right)+\gamma^{\prime \prime}+z^{\prime} C-i \epsilon\right]^{2}} \times \\
& \sum_{i= \pm} \frac{\partial}{\partial \lambda} y_{i}(0)\left\{\delta\left(y_{i}(0)\right) \frac{y_{i}^{2}(0)}{\left|y_{i}^{2}(0) \mathcal{A}\left(\zeta, \zeta^{\prime}, \gamma^{\prime}, \kappa^{2}\right)-\mu^{2}\right|}+\right. \\
& \left.-\theta\left(y_{i}(0)\right) 2 \mu^{2} \frac{y_{i}(0)}{\left(y_{i}^{2}(0) \mathcal{A}\left(\zeta, \zeta^{\prime}, \gamma^{\prime}, \kappa^{2}\right)-\mu^{2}\right)\left|y_{i}^{2}(0) \mathcal{A}\left(\zeta, \zeta^{\prime}, \gamma^{\prime}, \kappa^{2}\right)-\mu^{2}\right|}\right\}= \\
& =\int_{-\infty}^{\infty} d \gamma^{\prime \prime} \int_{-1}^{1} d z^{\prime} \frac{\mathcal{Q}^{\prime}\left(z, z_{i} ; \gamma^{\prime}, \gamma^{\prime \prime}, z^{\prime}, \zeta, \zeta^{\prime}, \cos \theta, \mu^{2}\right)}{\left[\gamma+z^{2} m^{2}+\kappa^{2}\left(1-z^{2}\right)+\gamma^{\prime \prime}+z^{\prime} C-i \epsilon\right]^{2}}
\end{aligned}
$$

where

$$
\begin{aligned}
& \mathcal{Q}^{\prime}\left(z, z_{i} ; \gamma^{\prime}, \gamma^{\prime \prime}, z^{\prime}, \zeta, \zeta^{\prime}, \mu^{2}\right)=\theta\left(\frac{1+z}{1+\zeta^{\prime}-z_{i} \zeta}-\frac{z^{\prime}}{\zeta}\right) \frac{\theta\left(z^{\prime}\right) \theta(\zeta)-\theta\left(-z^{\prime}\right) \theta(-\zeta)}{z^{\prime}} \times \\
& \Lambda\left(z, \frac{z^{\prime}}{\zeta}, \zeta, \zeta^{\prime} ; \gamma^{\prime}, \gamma^{\prime \prime} ; z_{i}, \mu^{2}\right)
\end{aligned}
$$

with

$$
\begin{aligned}
& \Lambda\left(z, \frac{z^{\prime}}{\zeta}, \zeta, \zeta^{\prime} ; \gamma^{\prime}, \gamma^{\prime \prime} ; z_{i}, \mu^{2}\right)=\sum_{i= \pm} \frac{\partial}{\partial \lambda} y_{i}(0)\left\{\delta\left(y_{i}(0)\right) \frac{y_{i}^{2}(0)}{\left|y_{i}^{2}(0) \mathcal{A}\left(\zeta, \zeta^{\prime}, \gamma^{\prime}, \kappa^{2}\right)-\mu^{2}\right|}+\right. \\
& \left.-\theta\left(y_{i}(0)\right) \frac{2 \mu^{2} y_{i}(0)}{\left(y_{i}^{2}(0) \mathcal{A}\left(\zeta, \zeta^{\prime}, \gamma^{\prime}, \kappa^{2}\right)-\mu^{2}\right)\left|y_{i}^{2}(0) \mathcal{A}\left(\zeta, \zeta^{\prime}, \gamma^{\prime}, \kappa^{2}\right)-\mu^{2}\right|}\right\}
\end{aligned}
$$


and

$$
\begin{aligned}
& y_{ \pm}(0)=\frac{1}{2 \mathcal{A}\left(\zeta, \zeta^{\prime}, \gamma^{\prime}, \kappa^{2}\right)} \times \\
& {\left[-\mathcal{B}\left(z, z^{\prime}, z_{i}, \zeta, \zeta^{\prime}, \gamma^{\prime}, \gamma^{\prime \prime}, \mu^{2}, 0\right) \pm \sqrt{\mathcal{B}^{2}\left(z, z^{\prime}, z_{i}, \zeta, \zeta^{\prime}, \gamma^{\prime}, \gamma^{\prime \prime}, \mu^{2}, 0\right)-4 \mu^{2} \mathcal{A}\left(\zeta, \zeta^{\prime}, \gamma^{\prime}, \kappa^{2}\right)}\right]} \\
& \frac{\partial}{\partial \lambda} y_{i}(0)=\mp \frac{\left(1+\zeta^{\prime}-z_{i} \zeta\right)}{(1+z)} \frac{y_{ \pm}(0)}{\sqrt{\mathcal{B}^{2}\left(z, z^{\prime}, z_{i}, \zeta, \zeta^{\prime}, \gamma^{\prime}, \gamma^{\prime \prime}, \mu^{2}, 0\right)-4 \mu^{2} \mathcal{A}\left(\zeta, \zeta^{\prime}, \gamma^{\prime}, \kappa^{2}\right)}}(\mathrm{B} 39)
\end{aligned}
$$

Let us recall that $y_{i}(0)$ still depends upon $z, z_{i}, \zeta$ and $\zeta^{\prime}$, and therefore the proper changes, dictated by the arguments of $\mathcal{Q}^{\prime}$, must be performed in order to get the final expression for $V_{s}^{(L)}$. The necessity of real solutions for getting non vanishing Nakanishi amplitude imposes a constraint on the discriminant of Eq. (B35), viz

$$
\begin{aligned}
& \mathcal{B}^{2}\left(z, z^{\prime}, z_{i}, \zeta, \zeta^{\prime}, \gamma^{\prime}, \gamma^{\prime \prime}, \mu^{2}, 0\right)-4 \mu^{2} \mathcal{A}\left(\zeta, \zeta^{\prime}, \gamma^{\prime}, \kappa^{2}\right)= \\
& =\left(\mu^{2}+\gamma^{\prime}-\gamma^{\prime \prime} \frac{\zeta}{z^{\prime}}\right)^{2}-4 \mu^{2}\left[\zeta^{\prime 2} \frac{M^{2}}{4}+\kappa^{2}\left(1+\zeta^{2}\right)+\gamma^{\prime}\right] \geq 0
\end{aligned}
$$

More constraints can be obtained from the positivity of the solutions $y_{i}(0)$. In particular one has

- if $\mathcal{A}\left(\zeta, \zeta^{\prime}, \gamma^{\prime}, \kappa^{2}\right) \geq 0$, then one has to consider $\mathcal{B}\left(z, z^{\prime}, z_{i}, \zeta, \zeta^{\prime}, \gamma^{\prime}, \gamma^{\prime \prime}, \mu^{2}, 0\right) \leq$ $-2 \mu \sqrt{\mathcal{A}\left(\zeta, \zeta^{\prime}, \gamma^{\prime}, \kappa^{2}\right)}$. In this case, two solution for $y_{i}(0)$ are allowed;

- if $\mathcal{A}\left(\zeta, \zeta^{\prime}, \gamma^{\prime}, \kappa^{2}\right)<0$, then $\mathcal{B}\left(z, z^{\prime}, z_{i}, \zeta, \zeta^{\prime}, \gamma^{\prime}, \gamma^{\prime \prime}, \mu^{2}, 0\right) \geq 0$. Only one solution is allowed.

In conclusion, from Eq. (B24) and using Eq. (B36), one has

$$
\begin{aligned}
& V_{s}^{(L)}\left(\gamma, z ; \gamma_{i}, z_{i}, \gamma^{\prime \prime}, \zeta, \zeta^{\prime}, \cos \theta\right)=-\frac{g^{2}}{2(4 \pi)^{2}} \int_{-\infty}^{\infty} d \gamma^{\prime} \int_{-1}^{1} d z^{\prime} \times \\
& \frac{1}{\left[\gamma+z^{2} m^{2}+\kappa^{2}\left(1-z^{2}\right)+\gamma^{\prime}+z^{\prime}\left(\frac{M^{2}}{2} z z_{i}+2 \cos \theta \sqrt{\gamma \gamma_{i}}\right)-i \epsilon\right]^{2}} \times \\
& {\left[\frac{(1+z)}{\left(1+\zeta^{\prime}-z_{i} \zeta\right)} \theta\left(\zeta^{\prime}-z-z_{i} \zeta\right) \mathcal{Q}^{\prime}\left(z, z_{i} ; \gamma^{\prime \prime}, \gamma^{\prime}, z^{\prime}, \zeta, \zeta^{\prime}, \mu^{2}\right)+\right.} \\
& \left.+\frac{(1-z)}{\left(1-\zeta^{\prime}+z_{i} \zeta\right)} \theta\left(z-\zeta^{\prime}+z_{i} \zeta\right) \mathcal{Q}^{\prime}\left(-z,-z_{i} ; \gamma^{\prime \prime}, \gamma^{\prime}, z^{\prime} \zeta,-\zeta^{\prime}, \mu^{2}\right)\right]
\end{aligned}
$$

where for the sake of notation adopted in Sect. IV the change $\gamma^{\prime \prime} \rightarrow \gamma^{\prime}$ has been performed.

[1] E.E. Salpeter, H.A. Bethe, Phys. Rev. 84, 1232 (1951). 
[2] N. Nakanishi, Prog. Theor. Phys. Suppl. 43, 1 (1969); 95, 1 (1988).

[3] G.C. Wick, Phys. Rev. 96, 1124 (1954).

[4] S.M. Dorkin, L.P. Kaptari, C. Ciofi degli Atti, B. Kämpfer, Few Body Syst. 49, 233 (2011).

[5] F. Gross and A. Stadler, Few Body Syst. 49, 91 (2011).

[6] C. D. Roberts, Prog. Part. Nucl. Phys. 61, 50 (2008).

[7] K Nishijima, Prog. Theor. Phys. 14, 203 (1955).

[8] C. Schwartz and C. Zemach, Phys. Rev. 141, 1454 (1966).

[9] S. G. Bondarenko, V. V. Burov, M. Beyer and S. M. Dorkin, Few-Body Syst. 26, 185 (1999); S. M. Dorkin, M. Beyer, S. S. Semikh and L. P. Kaptari, Few-Body Syst. 42, 1 (2008).

[10] N. Nakanishi, Phys. Rev. 127, 1380 (1962); Phys. Rev. 130, 1230 (1963) Graph Theory and Feynman Integrals (Gordon and Breach, New York, 1971).

[11] V.A. Karmanov and J. Carbonell, Eur. Phys. J. A27, 1 (2006).

[12] J. Carbonell, V.A. Karmanov, Eur. Phys. J. A27, 11 (2006).

[13] K. Kusaka, A.G. Williams, Phys. Rev. D 51, 7026 (1995); K. Kusaka, K. Simpson, A.G. Williams, Phys. Rev. D 56, 5071 (1997).

[14] N. Nakanishi,Phys. Rev. 133, B214 (1964); Phys. Rev. 135, B1224 (1964).

[15] S. J. Brodsky, C.-R. Ji and M. Sawicki, Phys. Rev. D 32, 1530 (1985).

[16] Dae Sung Hwang and V. A. Karmanov, Nucl. Phys. 696, 413 (2004).

[17] V. Sauli, J. Adam, Phys. Rev.D 67, 085007 (2003); V. Sauli, J. Phys. G 35, 035005 (2008)

[18] J. Carbonell, V.A. Karmanov, M. Mangin-Brinet, Eur. Phys. J. A39, 53 (2009).

[19] J. Carbonell, V.A. Karmanov Eur. Phys. J. A46, (2010) 387.

[20] J. Carbonell and V. A. Karmanov, Few-body Syst. 49, 205 (2011).

[21] P. C. Magalhães, M. R. Robilotta, K. S. F. F. Guimarães, T. Frederico, W. de Paula, I. Bediaga, A. C. dos Reis, "Towards three-body unitarity in $D^{+} \rightarrow K^{-} \pi^{+} \pi^{+}$, arXiv:1105.5120v2 [hep-ph], in press Phys. Rev. D.

[22] G. F. Giudice, R. Rattazzi, J. D. Wells, Nuc. Phys. B 630, 293 (2002).

[23] J. Carbonell, B. Desplanques, V.A. Karmanov and J.F. Mathiot, Phys. Reports, 300, 215 (1998).

[24] J. H. O. Sales, T. Frederico, B. V. Carlson, P. U. Sauer, Phys. Rev. C 61, 044003 (2000).

[25] J. H. O. Sales, T. Frederico, B. V. Carlson, P. U. Sauer, Phys. Rev. C 63, 064003 (2001).

[26] T. Frederico, J. H. O. Sales, B.V. Carlson and P.U. Sauer Nucl. Phys. A 737, 260c (2004). 
[27] J. A. O. Marinho, T. Frederico, P. U. Sauer, Phys. Rev. D 76, 096001 (2007).

[28] J.A.O. Marinho, T. Frederico, E. Pace, G. Salme, P. U. Sauer, Phys. Rev. D 77, 116010 (2008).

[29] T. Frederico and G. Salmè, Few-body Syst. 49, 163 (2011).

[30] R.E. Cutkosky, Phys. Rev. 96, 1135 (1954).

[31] S. J. Brodsky, H. C. Pauli and S. S. Pinsky, Phys. Rep. 301, 299 (1998) arXiv:hep-ph/9705477.

[32] S. J. Brodsky, C. D. Roberts, R. Shrock and P. C. Tandy, Phys. Rev. C 82, 022201 (2010).

[33] P. A. M. Dirac, Rev. Mod. Phys. 21, 392 (1949).

[34] R. M. Woloshyn and A. D. Jackson, Nucl. Phys. B 64, 269 (1973).

[35] C. Itzykson, J.B. Zuber "Quantum Field Theory", Dover Publications (2006).

[36] B.L.G. Bakker, J.K. Boomsma and C.-R. Ji, Phys. Rev. D 75, 065010 (2007).

[37] J.R. Cooke, G.A. Miller and D.R. Phillips, Phys. Rev. C 61, 064005 (2000).

[38] M. Mangin-Brinet and J. Carbonell, Phys. Lett. B 474, 237 (2000).

[39] F. Gross "Relativistic Quantum Mechanics and Field Theory",John Wiley and Sons, NewYork (1993).

[40] N.E. Ligterink and B.L.G. Bakker, Phys. Rev. D 52, 5954 (1995).

[41] M. Sawicki, Phys. Rev. D 32, 2666 (1985); D 33, 1103 (1986).

[42] A. Castro Neto, F. Guinea, N. M. R.Peres, K. Novoselov, and A. Geim, Rev. Mod. Phys. 81, 109 (2009).

[43] N. M. R. Peres, Rev. Mod. Phys. 82, 2673 (2010). 